\title{
Representations of the quantum Teichmüller space and invariants of surface diffeomorphisms
}

\author{
FRANCIS BONAHON \\ XIAOBO LiU
}

\begin{abstract}
We investigate the representation theory of the polynomial core $\mathcal{T}_{S}^{q}$ of the quantum Teichmüller space of a punctured surface $S$. This is a purely algebraic object, closely related to the combinatorics of the simplicial complex of ideal cell decompositions of $S$. Our main result is that irreducible finite-dimensional representations of $\mathcal{T}_{S}^{q}$ are classified, up to finitely many choices, by group homomorphisms from the fundamental group $\pi_{1}(S)$ to the isometry group of the hyperbolic 3-space $\mathbb{H}^{3}$. We exploit this connection between algebra and hyperbolic geometry to exhibit invariants of diffeomorphisms of $S$.
\end{abstract}

57R56; 57M50, 20G42

This work finds its motivation in the emergence of various conjectural connections between topological quantum field theory and hyperbolic geometry, such as the now famous Volume Conjecture of Rinat Kashaev [22], and Hitochi Murakami and Jun Murakami [28]. For a hyperbolic link $L$ in the 3-sphere $S^{3}$, this conjectures relates the hyperbolic volume of the complement $S^{3}-L$ to the asymptotic behavior of the $N$-th colored Jones polynomial $J_{L}^{N}\left(\mathrm{e}^{2 \pi \mathrm{i} / N}\right)$ of $L$, evaluated at the primitive $N$-th root of unity $e^{2 \pi \mathrm{i} / N}$. At this point, the heuristic evidence (Kashaev [22], Murakami, Murakami, Okamoto, Takata and Yokota [29], and Yokota [43; 44]) for the Volume Conjecture is based on the observation $[22 ; 28]$ that the $N$-th Jones polynomial can be computed using an explicit R-matrix whose asymptotic behavior is related to Euler's dilogarithm function, which is well-known to give the hyperbolic volume of an ideal tetrahedron in $\mathbb{H}^{3}$ in terms of the cross-ratio of its vertices. We wanted to establish a more conceptual connection between the two points of view, namely between quantum algebra and 3-dimensional hyperbolic geometry.

We investigate such a relationship, provided by the quantization of the Teichmüller space of a surface, as developed by Rinat Kashaev [23], Leonid Chekhov and Vladimir Fock [12]. More precisely, we follow the exponential version of the Chekhov-Fock approach. This enables us to formulate our discussion in terms of non-commutative algebraic geometry and finite-dimensional representations of algebras, instead of Lie algebras and self-adjoint operators of Hilbert spaces. This may be physically less 
relevant, but this point of view is better adapted to the problems that we have in mind. The mathematical foundations of this non-commutative algebraic geometric point of view are rigorously established by Liu in [25].

More precisely, let $S$ be a surface of finite topological type, with genus $g$ and with $p \geqslant 1$ punctures. An ideal triangulation of $S$ is a proper 1-dimensional submanifold whose complementary regions are infinite triangles with vertices at infinity, namely at the punctures. For an ideal triangulation $\lambda$ and a number $q=\mathrm{e}^{\pi \mathrm{i} \hbar} \in \mathbb{C}$, the ChekhovFock algebra $\mathcal{T}_{\lambda}^{q}$ is the algebra over $\mathbb{C}$ defined by generators $X_{1}^{ \pm 1}, X_{2}^{ \pm 1}, \ldots, X_{n}^{ \pm 1}$ associated to the components of $\lambda$ and by relations $X_{i} X_{j}=q^{2 \sigma_{i j}} X_{j} X_{i}$, where the $\sigma_{i j}$ are integers determined by the combinatorics of the ideal triangulation $\lambda$. This algebra has a well-defined fraction division algebra $\hat{\mathcal{T}}_{\lambda}^{q}$. In concrete terms, $\mathcal{T}_{\lambda}^{q}$ consists of the formal Laurent polynomials in variables $X_{i}$ satisfying the skew-commutativity relations $X_{i} X_{j}=q^{2 \sigma_{i j}} X_{j} X_{i}$, while its fraction algebra $\hat{\mathcal{T}}_{\lambda}^{q}$ consists of formal rational fractions in the $X_{i}$ satisfying the same relations.

As one moves from one ideal triangulation $\lambda$ to another $\lambda^{\prime}$, Chekhov and Fock $[12 ; 15$; 16] (see also [25]) introduce coordinate change isomorphisms $\Phi_{\lambda \lambda^{\prime}}^{q}: \hat{\mathcal{T}}_{\lambda^{\prime}}^{q} \rightarrow \hat{\mathcal{T}}_{\lambda}^{q}$ which satisfy the natural property that $\Phi_{\lambda^{\prime \prime} \lambda^{\prime}}^{q} \circ \Phi_{\lambda^{\prime} \lambda}^{q}=\Phi_{\lambda^{\prime \prime} \lambda}^{q}$ for every ideal triangulations $\lambda, \lambda^{\prime}, \lambda^{\prime \prime}$. In a triangulation independent way, this associates to the surface $S$ the algebra $\hat{\mathcal{T}}_{S}^{q}$ defined as the quotient of the family of all $\hat{\mathcal{T}}_{\lambda}^{q}$, with $\lambda$ ranging over ideal triangulations of the surface $S$, by the equivalence relation that identifies $\hat{\mathcal{T}}_{\lambda}^{q}$ and $\hat{\mathcal{T}}_{\lambda^{\prime}}^{q}$ by the coordinate change isomorphism $\Phi_{\lambda \lambda^{\prime}}^{q}$. By definition, $\hat{\mathcal{T}}_{S}^{q}$ is the quantum Teichmüller space of the surface $S$.

This construction and definition are motivated by the case where $q=1$, in which case $\hat{\mathcal{T}}_{\lambda}^{1}$ is just the algebra $\mathbb{C}\left(X_{1}, X_{2}, \ldots, X_{n}\right)$ of rational functions in $n$ commuting variables. Bill Thurston associated to each ideal triangulation a global coordinate system for the Teichmüller space $\mathcal{T}(S)$ consisting of all isotopy classes of complete hyperbolic metrics on $S$. Given two ideal triangulations $\lambda$ and $\lambda^{\prime}$, the corresponding coordinate changes are rational, so that there is a well-defined notion of rational functions on $\mathcal{T}(S)$. For a given ideal triangulation $\lambda$, Thurston's shear coordinates provide a canonical isomorphism between the algebra of rational functions on $\mathcal{T}(S)$ and $\mathbb{C}\left(X_{1}, X_{2}, \ldots, X_{n}\right) \cong \hat{\mathcal{T}}_{\lambda}^{1}$. It turns out that the $\Phi_{\lambda \lambda^{\prime}}^{1}$ are just the corresponding coordinate changes. Therefore, the quantum Teichmüller space $\hat{\mathcal{T}}_{S}^{q}$ is a (non-commutative) deformation of the algebra of rational functions on the Teichmüller space $\mathcal{T}(S)$.

Although the construction of $\hat{\mathcal{T}}_{S}^{q}$ was motivated by the geometry, a result of Hua Bai [1] shows that it actually depends only on the combinatorics of ideal triangulations. Indeed, once we fix the definition of the Chekhov-Fock algebras $\mathcal{T}_{\lambda}^{q}$, the coordinate change isomorphisms $\Phi_{\lambda \lambda^{\prime}}^{q}: \hat{\mathcal{T}}_{\lambda^{\prime}}^{q} \rightarrow \widehat{\mathcal{T}}_{\lambda}^{q}$ are uniquely determined if we require them to 
satisfy a certain number of natural conditions, a typical one being the locality condition: if $\lambda$ and $\lambda^{\prime}$ share a component $\lambda_{i}$ as well as any component of $\lambda$ that is adjacent to $\lambda_{i}$, then $\Phi_{\lambda \lambda^{\prime}}^{q}$ must respect the corresponding generator $X_{i}$.

A standard method to move from abstract algebraic constructions to more concrete applications is to consider finite-dimensional representations. In the case of algebras, this means algebra homomorphisms valued in the algebra $\operatorname{End}(V)$ of endomorphisms of a finite-dimensional vector space $V$ over $\mathbb{C}$. Elementary considerations show that these can exist only when $q$ is a root of unity.

Theorem 1 Suppose that $q^{2}$ is a primitive $N$-th root of unity, and consider the Chekhov-Fock algebra $\mathcal{T}_{\lambda}^{q}$ associated to an ideal triangulation $\lambda$. Every irreducible finite-dimensional representation of $\mathcal{T}_{\lambda}^{q}$ has dimension $N^{3 g+p-3}$ if $N$ is odd, and $N^{3 g+p-3} / 2^{g}$ if $N$ is even, where $g$ is the genus of the surface $S$ and where $p$ is its number of punctures. Up to isomorphism, such a representation is classified by:

(1) a non-zero complex number $x_{i} \in \mathbb{C}^{*}$ associated to each edge of $\lambda$;

(2) a choice of an $N$-th root for each of $p$ explicit monomials in the numbers $x_{i}$;

(3) when $N$ is even, a choice of square root for each of $2 g$ explicit monomials in the numbers $x_{i}$.

Conversely, any such data can be realized by an irreducible finite-dimensional representation of $\mathcal{T}_{\lambda}^{q}$.

The numbers $x_{i} \in \mathbb{C}^{*}$ appearing in the classification of a representation $\rho: \mathcal{T}_{\lambda}^{q} \rightarrow$ $\operatorname{End}(V)$ are characterized by the property that $\rho\left(X_{i}^{N}\right)=x_{i} \operatorname{Id}_{V}$ for the corresponding generator $X_{i}$ of $\mathcal{T}_{\lambda}^{q}$. Theorem 1 is proved in Section 4. The main step in the proof, which has a strong topological component, is to determine the algebraic structure of the algebra $\mathcal{T}_{\lambda}^{q}$ and is completed in Section 3 after preliminary work in Section 2. Another important feature of Theorem 1 is the way it is stated, which closely ties the classification to the combinatorics of the ideal triangulation $\lambda$ in $S$ and counterbalances the fact that the structure results for $\mathcal{T}_{\lambda}^{q}$ are not very explicit.

Theorem 1 shows that the Chekhov-Fock algebra has a rich representation theory. Unfortunately, for dimension reasons, its fraction algebra $\hat{\mathcal{T}}_{\lambda}^{q}$ and, consequently, the quantum Teichmüller space $\widehat{\mathcal{T}}_{S}^{q}$ cannot have any finite-dimensional representation. This leads us to introduce the polynomial core $\mathcal{T}_{S}^{q}$ of the quantum Teichmüller space $\widehat{\mathcal{T}}_{S}^{q}$, defined as the family $\left\{\mathcal{T}_{\lambda}^{q}\right\}_{\lambda \in \Lambda(S)}$ of all Chekhov-Fock algebras $\mathcal{T}_{\lambda}^{q}$, considered as subalgebras of $\hat{\mathcal{T}}_{S}^{q}$, as $\lambda$ ranges over the set $\Lambda(S)$ of all isotopy classes of ideal triangulations of the surface $S$. In Section 6, we introduce and analyze the consistency 
of a notion of representation of the polynomial core, consisting of the data of representations $\rho_{\lambda}: \mathcal{T}_{\lambda}^{q} \rightarrow \operatorname{End}(V)$ for all $\lambda \in \Lambda(S)$ that behave well under the coordinate changes $\Phi_{\lambda \lambda^{\prime}}^{q}$.

We now jump from the purely algebraic representation theory of the polynomial core $\mathcal{T}_{S}^{q}$ to 3-dimensional hyperbolic geometry. Theorem 1 says that, up to a finite number of choices, an irreducible representation of $\mathcal{T}_{\lambda}^{q}$ is classified by certain numbers $x_{i} \in \mathbb{C}^{*}$ associated to the edges of the ideal triangulation $\lambda$ of $S$. There is a classical geometric object which is also associated to $\lambda$ with the same edge weights $x_{i}$. Namely, we can consider in the hyperbolic 3-space $\mathbb{H}^{3}$ the pleated surface that has pleating locus $\lambda$, that has shear parameter along the $i$-th edge of $\lambda$ equal to the real part of $\log x_{i}$, and that has bending angle along this edge equal to the imaginary part of $\log x_{i}$. In turn, this pleated surface has a monodromy representation, namely a group homomorphism from the fundamental group $\pi_{1}(S)$ to the group $\operatorname{Isom}^{+}\left(\mathbb{M}^{3}\right) \cong \mathrm{PSL}_{2}(\mathbb{C})$ of orientationpreserving isometries of $\mathbb{T}^{3}$. This construction associates to a representation of the Chekhov-Fock algebra $\mathcal{T}_{\lambda}^{q}$ a group homomorphism $r: \pi_{1}(S) \rightarrow \operatorname{PSL}_{2}(\mathbb{C})$, welldefined up to conjugation by an element of $\mathrm{PSL}_{2}(\mathbb{C})$.

It turns out that, for a suitable choice of $q$, this construction is well-behaved under coordinate changes. The fact that $q^{2}$ is a primitive $N$-th root of unity implies that $q^{N}= \pm 1$, but the following result requires that $q^{N}=(-1)^{N+1}$. This is automatically satisfied if $N$ is even.

Theorem 2 Let $q$ be a primitive $N$-th root of $(-1)^{N+1}$, for instance $q=-\mathrm{e}^{\pi \mathrm{i} / N}$. If $\rho=\left\{\rho_{\lambda}: \mathcal{T}_{\lambda}^{q} \rightarrow \operatorname{End}(V)\right\}_{\lambda \in \Lambda(S)}$ is a finite-dimensional irreducible representation of the polynomial core $\mathcal{T}_{S}^{q}$ of the quantum Teichmüller space $\hat{\mathcal{T}}_{S}^{q}$, the representations $\rho_{\lambda}$ induce the same monodromy homomorphism $r_{\rho}: \pi_{1}(S) \rightarrow \operatorname{PSL}_{2}(\mathbb{C})$.

Theorem 2 is essentially equivalent to the property that, for the choice of $q$ indicated, the pleated surfaces respectively associated to the representations $\rho_{\lambda}: \mathcal{T}_{\lambda}^{q} \rightarrow \operatorname{End}(V)$ and $\rho_{\lambda} \circ \Phi_{\lambda \lambda^{\prime}}^{q}: \mathcal{T}_{\lambda^{\prime}}^{q} \rightarrow \operatorname{End}(V)$ have (different pleating loci but) the same monodromy representation $r_{\rho}: \pi_{1}(S) \rightarrow \mathrm{PSL}_{2}(\mathbb{C})$. Its proof splits into two parts: a purely algebraic computation in Section 7, which is based on the quantum binomial formula and is borrowed from a remark in Chekhov and Fock [16], relates the quantum case to the non-quantum case where $q=1$; a more geometric part in Section 8 is completely centered on the non-quantum situation and uses pleated surfaces in hyperbolic 3-space.

The homomorphism $r_{\rho}$ is the hyperbolic shadow of the representation $\rho$. Not every homomorphism $r: \pi_{1}(S) \rightarrow \mathrm{PSL}_{2}(\mathbb{C})$ is the hyperbolic shadow of a representation of the polynomial core, but many of them are: 
Theorem 3 An injective homomorphism $r: \pi_{1}(S) \rightarrow \mathrm{PSL}_{2}(\mathbb{C})$ is the hyperbolic shadow of a finite number of irreducible finite-dimensional representations of the polynomial core $\mathcal{T}_{S}^{q}$, up to isomorphism. More precisely, this number of representations is equal to $2^{l} N^{p}$ if $N$ is odd, and $2^{2 g+l} N^{p}$ if $N$ is even, where $g$ is the genus of $S$, $p$ is its number of punctures, and $l$ is the number of ends of $S$ whose image under $r$ is loxodromic.

As an application of this machinery, we construct new and still mysterious invariants of (isotopy classes of) surface diffeomorphisms, by using Theorems 2 and 3 to go back and forth between hyperbolic geometry and representations of the polynomial core $\mathcal{T}_{S}^{q}$.

Let $\varphi$ be a diffeomorphism of the surface $S$. Suppose in addition that $\varphi$ is homotopically aperiodic (also called homotopically pseudo-Anosov), so that its (3-dimensional) mapping torus $M_{\varphi}$ admits a complete hyperbolic metric. The hyperbolic metric of $M_{\varphi}$ gives an injective homomorphism $r_{\varphi}: \pi_{1}(S) \rightarrow \mathrm{PSL}_{2}(\mathbb{C})$ such that $r_{\varphi} \circ \varphi^{*}$ is conjugate to $r_{\varphi}$, where $\varphi^{*}$ is the isomorphism of $\pi_{1}(S)$ induced by $\varphi$.

The diffeomorphism $\varphi$ also acts on the quantum Teichmüller space and on its polynomial core $\mathcal{T}_{S}^{q}$. In particular, it acts on the set of representations of $\mathcal{T}_{S}^{q}$ and, because $r_{\varphi} \circ \varphi^{*}$ is conjugate to $r_{\varphi}$, it sends a representation with hyperbolic shadow $r_{\varphi}$ to another representation with shadow $r_{\varphi}$. Actually, when $N$ is odd, there is a preferred representation $\rho_{\varphi}$ of $\mathcal{T}_{S}^{q}$ which is fixed by the action of $\varphi$, up to isomorphism. This statement means that, for every ideal triangulation $\lambda$, we have a representation $\rho_{\lambda}: \mathcal{T}_{\lambda}^{q} \rightarrow \operatorname{End}(V)$ of dimension $N^{3 g+p-3}$ and an isomorphism $L_{\varphi}^{q}$ of $V$ such that

$$
\rho_{\varphi(\lambda)} \circ \Phi_{\varphi(\lambda) \lambda}(X)=L_{\varphi}^{q} \cdot \rho_{\lambda}(X) \cdot\left(L_{\varphi}^{q}\right)^{-1}
$$

in $\operatorname{End}(V)$ for every $X \in \mathcal{T}_{\lambda}^{q}$, for a suitable interpretation of the left hand side of the equation.

Theorem 4 Let $N$ be odd. Up to conjugation and up to multiplication by a constant, the isomorphism $L_{\varphi}^{q}$ depends only on the homotopically aperiodic diffeomorphism $\varphi: S \rightarrow S$ and on the primitive $N$-th root $q$ of 1 .

Note that $L_{\varphi}^{q}$ is an isomorphism of a vector space of very large dimension $N^{3 g+p-3}$, and consequently encodes a lot of information. Extracting invariants from $L_{\varphi}^{q}$ provides simpler invariants of $\varphi$, such as the projectivized spectrum of $L_{\varphi}^{q}$. We can also normalize $L_{\varphi}$ so that it has determinant 1 , in which case its trace gives an invariant of $\varphi$ defined up to multiplication by a root of unity.

Explicit computations of these invariants in certain examples are provided in [26]. 
As is often the case with invariants from Topological Quantum Field Theory, the invariants extracted from $L_{\varphi}^{q}$ are by themselves unlikely to have many practical applications. What is more interesting is their connections with other combinatorial and geometric objects.

As this work was being developed, the type of functions occurring in explicit computations hinted at a connection between the invariant of Theorem 4, the Kashaev $6 j$-symbols developed in [21], and the link invariants introduced by Kashaev [21; 22], Baseilhac and Benedetti [4; 5; 6; 7]; see also Murakami-Murakami [28]. This connection has now been elucidated by the authors and Hua Bai $[2 ; 3]$. Whereas the current article focuses on irreducible representations, [3] investigates another type of representations of the quantum Teichmüller space, called local representations, which are somewhat simpler to analyze and more closely connected to the combinatorics of ideal triangulations. The classification of these local representations follows the same lines as the classification of irreducible representations, in terms of complex edge weights for ideal triangulations. An analogue of Theorem 4 then associates to a homotopically aperiodic diffeomorphism $\varphi: S \rightarrow S$ a large matrix $K_{\varphi}^{q}$, well-defined up to conjugation and multiplication by a root of unity. If one decomposes a local representation into its irreducible components, the invariant $L_{\varphi}^{q}$ of Theorem 4 and its generalizations discussed in Section 9 occur as building blocks of this $K_{\varphi}^{q}$. It can then be shown that the trace of $K_{\varphi}^{q}$ coincides with the invariant that, following the original insights of Kashaev, Baseilhac and Benedetti [6] associate to the hyperbolic metric of the mapping torus $M_{\varphi}$. A crucial step [2] is an explicit identification between the intertwining operator that a local representation associates to a diagonal exchange, and the $6 j$-symbols that Kashaev defines using the representation theory of the Weyl Hopf algebra.

The results of this paper are very reminiscent of a well-known principle in quantum algebra, which is that the representations of a quantum group are in correspondence with representations of the original non-quantum Lie group or algebra. It would also be conceptually helpful to establish a connection with the quantum group constructions of Bullock, Frohman and Kania-Bartoszyńska [10], and Frohman, Gelca and Lofaro [18; 19], or with the skein theory of Przytycki and Sikora [34; 35], and Turaev [42].

Acknowledgements It is a pleasure to thank Hua Bai, Leonid Chekhov and Bob Penner for very helpful conversations. In particular, this work originated from lectures given by Leonid Chekhov at USC, and the reader familiar with [16] will easily recognize our debt to the last paragraph of that paper. We are also grateful to Bob Guralnick, Chuck Lanski, Susan Montgomery and Lance Small for algebraic consultation, and to the referee for misprint hunting. 
This work was partially supported by the grant DMS-0103511 from the National Science Foundation.

\section{The Chekhov-Fock algebra}

Let $S$ be an oriented punctured surface of finite topological type, obtained by removing a finite set $\left\{v_{1}, v_{2}, \ldots, v_{p}\right\}$ from the closed oriented surface $\bar{S}$. Let $\lambda$ be an ideal triangulation of $S$, namely the intersection with $S$ of the 1-skeleton of a triangulation of $\bar{S}$ whose vertex set is equal to $\left\{v_{1}, v_{2}, \ldots, v_{p}\right\}$. In other words, $\lambda$ consists of finitely many disjoint simple arcs $\lambda_{1}, \lambda_{2}, \ldots, \lambda_{n}$ going from puncture to puncture and decomposing $S$ into finitely many triangles with vertices at infinity. Note that $n=-3 \chi(S)=6 g+3 p-6$, where $\chi(S)$ is the Euler characteristic of $S, g$ is the genus of $\bar{S}$ and $p$ is the number of punctures of $S$. In particular, we will require that $p \geqslant 3$ when $g=0$ to guarantee the existence of such ideal triangulations.

The complement $S-\lambda$ has $2 n$ spikes converging towards the punctures, and each spike is delimited by one $\lambda_{i}$ on one side and one $\lambda_{j}$ on the other side, with possibly $i=j$. For $i, j \in\{1, \ldots, n\}$, let $a_{i j}$ denote the number of spikes of $S-\lambda$ which are delimited on the left by $\lambda_{i}$ and on the right by $\lambda_{j}$ as one moves towards the end of the spike, and set

$$
\sigma_{i j}=a_{i j}-a_{j i}
$$

Note that $\sigma_{i j}$ can only belong to the set $\{-2,-1,0,+1,+2\}$, and that $\sigma_{j i}=-\sigma_{i j}$.

In the shear coordinates for Teichmüller space associated to the ideal triangulation $\lambda$, the antisymmetric bilinear form with matrix $\left(\sigma_{i j}\right)$ is closely related to the WeilPetersson closed 2-form on Teichmüller space $\mathcal{T}(S)$. Compare Papadopoulos and Penner $[31 ; 33]$ or Bonahon and Sözen [8;36], according to the type of Teichmüller space considered.

The Chekhov-Fock algebra associated to the ideal triangulation $\lambda$ is the algebra $\mathcal{T}_{\lambda}^{q}$ defined by the generators $X_{i}^{ \pm 1}$, with $i=1,2, \ldots, n$, and by the skew-commutativity relations

$$
X_{i} X_{j}=q^{2 \sigma_{i j}} X_{j} X_{i}
$$

for every $i, j$ (in addition to the relations $X_{i} X_{i}^{-1}=X_{i}^{-1} X_{i}=1$ ).

In particular, the Chekhov-Fock algebra $\mathcal{T}_{\lambda}^{q}$ is an iterated skew-polynomial algebra (see Cohn [13]) as well as a special type of multiparameter quantum torus (see Brown and Goodearl [9, Chapter I.2]). What is really important here is that its algebraic structure is tied to the combinatorics of the ideal triangulation $\lambda$ of the surface $S$.

We first analyze the algebraic structure of $\mathcal{T}_{\lambda}^{q}$. 


\section{The structure of the Weil-Petersson form}

The skew-commutativity coefficients $\sigma_{i j}$ form an antisymmetric matrix $\Sigma$, which defines an antisymmetric bilinear form $\sigma: \mathbb{Z}^{n} \times \mathbb{Z}^{n} \rightarrow \mathbb{Z}$. The key technical step to understanding the algebraic structure of $\mathcal{T}_{\lambda}^{q}$ is to classify the bilinear form $\sigma$ over the integers. Recall that two bilinear forms on $\mathbb{Z}^{n}$, with respective matrices $\Sigma$ and $\Sigma^{\prime}$, are equivalent over $\mathbb{Z}$ if there exists a base change matrix $A \in \mathrm{GL}_{n}(\mathbb{Z})$ such that $\Sigma^{\prime}=A \Sigma A^{\mathrm{t}}$.

Proposition 5 The antisymmetric bilinear form $\sigma: \mathbb{Z}^{n} \times \mathbb{Z}^{n} \rightarrow \mathbb{Z}$ is equivalent over $\mathbb{Z}$ to the block diagonal form consisting of $g$ blocks $\left(\begin{array}{cc}0 & -2 \\ 2 & 0\end{array}\right), k$ blocks $\left(\begin{array}{cc}0 & -1 \\ 1 & 0\end{array}\right)$ and $p$ blocks (0) on the diagonal, where $g$ is the genus of $S, p$ is its number of punctures, and $k=2 g+p-3$.

Proof Let $\Gamma \subset S$ be the graph dual to the ideal triangulation $\lambda$. Note that every vertex of $\Gamma$ is trivalent, and that $\Gamma$ is a deformation retract of $S$.

The coordinates of the $\mathbb{Z}^{n}$ considered above correspond to the components of $\lambda$. In a more intrinsic way, we consequently have a natural isomorphism between this $\mathbb{Z}^{n}$ and the group $\mathcal{H}(\lambda ; \mathbb{Z})$ of all assignments of integer weights to the components of $\lambda$ or, equivalently, to the edges of $\Gamma$. In particular, $\sigma$ is now an antisymmetric bilinear form on $\mathcal{H}(\lambda ; \mathbb{Z})$.

We first give a homological interpretation of $\mathcal{H}(\lambda ; \mathbb{Z})$ and $\sigma$, as is now somewhat standard when analyzing the Thurston intersection form on train tracks (see for instance Bonahon [8]).

Let $\widehat{\Gamma}$ be the oriented graph obtained from $\Gamma$ by keeping the same vertex set and by replacing each edge of $\Gamma$ by two oriented edges which have the same end points as the original edge, but which have opposite orientations. In particular, every vertex of $\widehat{\Gamma}$ now has valence 6 . There is a natural projection $p: \widehat{\Gamma} \rightarrow \Gamma$ which is one-to-one on the vertex set of $\hat{\Gamma}$ and two-to-one on the interior of the edges of $\hat{\Gamma}$.

There is a unique way to thicken $\widehat{\Gamma}$ to a surface $\widehat{S}$ such that:

(1) $\hat{S}$ deformation retracts to $\hat{\Gamma}$;

(2) as one goes around a vertex $\hat{v}$ of $\hat{\Gamma}$ in $\widehat{S}$, the orientations of the edges of $\widehat{\Gamma}$ adjacent to $\widehat{v}$ alternately point towards and away from $\widehat{v}$;

(3) the natural projection $p: \hat{\Gamma} \rightarrow \Gamma$ extends to a 2 -fold branched covering $\widehat{S} \rightarrow S$, branched along the vertex set of $\hat{\Gamma}$. 
Indeed, the last two conditions completely determine the local model for the inclusion of $\widehat{\Gamma}$ in $\widehat{S}$ near the vertices of $\hat{\Gamma}$.

Let $\tau: \widehat{S} \rightarrow \widehat{S}$ be the covering involution of the branched covering $p: \hat{S} \rightarrow S$. Note that $\tau$ respects $\hat{\Gamma}$, and reverses the orientation of its edges.

Lemma 6 There is a natural identification between $\mathcal{H}(\lambda ; \mathbb{Z}) \cong \mathbb{Z}^{n}$ and the subgroup of $H_{1}(\hat{S})=H_{1}(\hat{S} ; \mathbb{Z})$ consisting of those $\widehat{\alpha}$ such that $\tau_{*}(\widehat{\alpha})=-\widehat{\alpha}$.

Proof Every assignment $\alpha \in \mathcal{H}(\lambda ; \mathbb{Z})$ of weights to the edges of $\Gamma$ lifts to a $\tau$-invariant edge weight assignment $\hat{\alpha}$ for $\hat{\Gamma}$. Because the edges of $\hat{\Gamma}$ are oriented, $\hat{\alpha}$ actually defines a 1-chain on $\hat{\Gamma}$, whose boundary is equal to 0 because each edge $\hat{e}$ of $\hat{\Gamma}$ is paired with the edge $\tau(\hat{e})$ which has the same $\hat{\alpha}$-weight but such that $\partial \tau(\hat{e})=-\partial \hat{e}$. Therefore, we can interpret $\widehat{\alpha}$ as an element of $H_{1}(\widehat{\Gamma})$. Note that $\tau_{*}(\widehat{\alpha})=-\widehat{\alpha}$ since $\tau$ reverses the orientation of the edges of $\hat{\Gamma}$.

Conversely, every $\widehat{\alpha} \in H_{1}(\widehat{\Gamma})$ associates an integer weight to each edge of $\widehat{\Gamma}$, by considering its algebraic intersection number with an arbitrary point in the interior of the edge. If in addition $\tau_{*}(\hat{\alpha})=-\widehat{\alpha}$, this defines a $\tau$-invariant edge weight system on $\widehat{\Gamma}$, and therefore an element of $\mathcal{H}(\lambda ; \mathbb{Z})$.

This identifies $\mathcal{H}(\lambda ; \mathbb{Z})$ to the set of those $\widehat{\alpha} \in H_{1}(\hat{\Gamma})=H_{1}(\widehat{S})$ such that $\tau_{*}(\widehat{\alpha})=-\widehat{\alpha}$.

Lemma 7 If $\alpha, \beta \in \mathcal{H}(\lambda ; \mathbb{Z})$ correspond to $\hat{\alpha}, \hat{\beta} \in H_{1}(\widehat{S})$ as in Lemma 6, then $\sigma(\alpha, \beta)$ is equal to the algebraic intersection number $\hat{\alpha} \cdot \hat{\beta}$.

Proof It suffices to check this for each generator $\alpha_{i} \in \mathcal{H}(\lambda ; \mathbb{Z})$ assigning weight 1 to the edge $e_{i}$ of $\Gamma$ dual to the component $\lambda_{i}$ of $\lambda$, and weight 0 to the other edges of $\Gamma$. By definition, $\sigma\left(\alpha_{i}, \alpha_{j}\right)=\sigma_{i j}$ is equal to the number of times $e_{i}$ appears to the immediate left (as seen from the vertex) of $e_{j}$ at a vertex of $\Gamma$, minus the number of times $e_{i}$ appears to the immediate right of $e_{j}$. The corresponding homology class $\widehat{\alpha}_{i} \in H_{1}(\hat{S})$ is realized by the oriented closed curve $c_{i}$ that is the union of the two oriented edges of $\widehat{\Gamma}$ lifting $e_{i}$. In particular, $c_{i}$ and $c_{j}$ meet only at vertices of $\widehat{\Gamma}$ corresponding to common end points of the edges $e_{i}$ and $e_{j}$ in $\Gamma$. When $e_{i}$ is immediately to the left of $e_{j}$ at a vertex of $\Gamma$, it easily follows from our requirement that edge orientations alternately point in and out at the vertices of $\widehat{\Gamma}$ that the corresponding intersection between $c_{i}$ and $c_{j}$ has positive sign. Similarly, an end of $e_{i}$ which is immediately to the right of an end of $e_{j}$ contributes a -1 to the algebraic intersection number of $c_{i}$ with $c_{j}$. It follows that $\sigma\left(\alpha_{i}, \alpha_{j}\right)=c_{i} \cdot c_{j}=\widehat{\alpha}_{i} \cdot \hat{\alpha}_{j}$.

Therefore, $\sigma(\alpha, \beta)=\hat{\alpha} \cdot \hat{\beta}$ for every $\alpha, \beta \in \mathcal{H}(\lambda ; \mathbb{Z})$. 
We now analyze in more detail the branched covering $p: \hat{S} \rightarrow S$. We claim that the covering is trivial near the punctures of $S$. Indeed, if $\widehat{C}$ is a simple closed curve going around a puncture in $\widehat{S}$, the collapsing of $\widehat{S}$ to $\widehat{\Gamma}$ sends $\widehat{C}$ to a curve which is oriented by the orientation of the edges of $\Gamma$. This follows from our requirement that the orientations alternately point in and out at each vertex of $\hat{\Gamma}$. Since the covering involution $\tau$ reverses the orientation of the edges of $\widehat{\Gamma}$, we conclude that $\tau$ respects no puncture of $\hat{S}$. In other words, a puncture of $S$ lifts to two distinct punctures of $\hat{S}$, and the covering is trivial on a neighborhood of this puncture.

The branched covering $p: \hat{S} \rightarrow S$ is classified by a homomorphism $\pi_{1}(S-V) \rightarrow \mathbb{Z} / 2$, where $V$ is the set of branch points of $p$, namely the vertex set of $\Gamma$. Since the covering is trivial near the punctures of $S$, the corresponding class $H^{1}(S-V ; \mathbb{Z} / 2)$ is dual to the intersection with $S-V$ of a $1-$ submanifold $K \subset S$ with $\partial K=V$. One can arrange by surgery that $K$ consists only of arcs. Let $D \subset S$ be a disk containing $K$, and let $\widehat{D}$ be its preimage in $\widehat{S}$. The main point here is that the restriction $\widehat{S}-\widehat{D} \rightarrow S-D$ is now a trivial unbranched covering. In particular, $\widehat{S}$ is the union of $\widehat{D}$ and of two copies $\widehat{S}_{1}$ and $\widehat{S}_{2}$ of $S-D$.

The restriction of $p$ to $\widehat{D} \rightarrow D$ is a 2 -fold branched covering of a disk, with $4 g+$ $2 p-4$ branch points. It follows that $\hat{D}$ is a surface of genus $k=2 g+p-3$ with two boundary components. In addition, the covering involution is conjugate to a hyperelliptic involution of $\widehat{D}$, so that the induced homomorphism $\tau_{*}$ acts on $H_{1}(\widehat{D} ; \mathbb{Z})$ by multiplication by -1 .

Let $\widehat{D}^{0}, \widehat{S}_{1}^{0}$ and $\widehat{S}_{2}^{0}$ be the closed surfaces obtained by capping off the punctures and boundary components of $\widehat{D}, \widehat{S}_{1}$ and $\widehat{S}_{2}$, respectively. In addition, for $i=1,2, \ldots, p$, let $C_{i}$ be a small curve going counterclockwise around the $i$-th puncture in $S$, and let $\widehat{C}_{i 1}$ and $\widehat{C}_{i 2}$ be its respective lifts in $\widehat{S}_{1}$ and $\widehat{S}_{2}$. Then $H_{1}(\widehat{S})$ is isomorphic to $H_{1}\left(\widehat{D}^{0}\right) \oplus H_{1}\left(\widehat{S}_{1}^{0}\right) \oplus H_{1}\left(\widehat{S}_{2}^{0}\right) \oplus V$, where $V$ is the subgroup generated the $\widehat{C}_{i 1}$ and $\widehat{C}_{i 2}$. Note that the only relation between the homology classes of these $2 p$ curves is that they add up to 0 , so that $V \cong \mathbb{Z}^{2 p-1}$.

Lemma 6 identifies the space $\mathcal{H}(\lambda ; \mathbb{Z})$ of edge weight assignments to the subspace $\{\hat{\alpha} \in$ $\left.H_{1}(\widehat{S}) ; \tau_{*}(\widehat{\alpha})=-\widehat{\alpha}\right\}$. By construction, the isomorphism $\tau_{*}$ of $H_{1}(\widehat{S}) \cong H_{1}\left(\widehat{D}^{0}\right) \oplus$ $H_{1}\left(\widehat{S}_{1}^{0}\right) \oplus H_{1}\left(\hat{S}_{2}^{0}\right) \oplus V$ acts by multiplication by -1 on $H_{1}\left(\widehat{D}^{0}\right)$, exchanges the two factors $H_{1}\left(\widehat{S}_{1}^{0}\right) \cong H_{1}\left(\widehat{S}_{2}^{0}\right) \cong H_{1}(\bar{S})$, and acts on $V$ by transposing each pair $\left\{\widehat{C}_{i 1}, \widehat{C}_{i 2}\right\}$. (Recall that $\bar{S}$ is the closed surface such that $S=\bar{S}-\left\{v_{1}, v_{2}, \ldots, v_{p}\right\}$.) It follows that $\mathcal{H}(\lambda ; \mathbb{Z})$ consists of those $(x, y,-y, z)$ in $H_{1}(\widehat{S}) \cong H_{1}\left(\widehat{D}^{0}\right) \oplus H_{1}(\bar{S}) \oplus$ $H_{1}(\bar{S}) \oplus V$ such that $\tau_{*}(z)=-z$. This provides an isomorphism $\mathcal{H}(\lambda ; \mathbb{Z}) \cong H_{1}\left(\widehat{D}_{0}\right) \oplus$ $H_{1}(\bar{S}) \oplus W$, where $W=\left\{z \in V ; \tau_{*}(z)=-z\right\} \cong \mathbb{Z}^{p}$. 
By Lemma 7 , the bilinear form $\sigma$ is the restriction to $\mathcal{H}(\lambda ; \mathbb{Z})$ of the intersection form of $H_{1}(\widehat{S})$. We conclude that the three factors of the decomposition $\mathcal{H}(\lambda ; \mathbb{Z}) \cong$ $H_{1}\left(\widehat{D}_{0}\right) \oplus H_{1}(\bar{S}) \oplus W$ are orthogonal for $\sigma$, that the restriction of $\sigma$ to $H_{1}\left(\widehat{D}_{0}\right)$ is the intersection form of $\hat{D}_{0}$, that its restriction to $H_{1}(\bar{S})$ is twice the intersection form of $\bar{S}$ (because $y \in H_{1}(\bar{S})$ lifts to $\left.(0, y,-y, 0) \in H_{1}(\widehat{S}) \cong H_{1}\left(\widehat{D}_{0}\right) \oplus H_{1}(\bar{S}) \oplus H_{1}(\bar{S}) \oplus V\right)$, and that $\sigma$ is 0 on $W \cong \mathbb{Z}^{p}$.

Since $\widehat{D}_{0}$ and $\bar{S}$ are closed surfaces of respective genus $k$ and $g$, this concludes the proof of Proposition 5.

A consequence of Proposition 5 is that the kernel of the bilinear form $\sigma$, namely

$$
\operatorname{Ker} \sigma=\{\alpha \in \mathcal{H}(\lambda ; \mathbb{Z}) ; \forall \beta \in \mathcal{H}(\lambda ; \mathbb{Z}), \sigma(\alpha, \beta)=0\},
$$

is isomorphic to $\mathbb{Z}^{p}$. We can precise this result as follows. Index the punctures of $S$ from 1 to $p$. For $i=1, \ldots, p$ and $j=1, \ldots, n$, let $k_{i j} \in\{0,1,2\}$ denote the number of ends of the component $\lambda_{j}$ of $\lambda$ that converge to the $i-$ th puncture. Note that $\sum_{i=1}^{p}\left(k_{i 1}, k_{i 2}, \ldots, k_{i n}\right)=(2,2, \ldots, 2)$ since each $\lambda_{j}$ has two ends.

Lemma 8 In $\mathcal{H}(\lambda ; \mathbb{Z}) \cong \mathbb{Z}^{n}$, the kernel $\operatorname{Ker} \sigma$ is the abelian subgroup freely generated by the $p$ vectors $(1,1, \ldots, 1)$ and $\left(k_{i 1}, k_{i 2}, \ldots, k_{i n}\right)$, for $i=1, \ldots p-1$.

Proof Using the notation of the proof of Proposition 5, Ker $\sigma$ corresponds to the subspace $W$ of $\mathcal{H}(\lambda ; \mathbb{Z}) \cong H_{1}\left(\widehat{D}_{0}\right) \oplus H_{1}(\bar{S}) \oplus W$. We need to backtrack through the definition of $W$.

Recall that, for each $i=1, \ldots, p$, we picked an oriented closed curve $C_{i}$ going counterclockwise around the $i$-th puncture of $S$, and that we lifted it to curves $\widehat{C}_{i 1}$ and $\widehat{C}_{i 2}$ in $\widehat{S}_{1}$ and $\widehat{S}_{2}$, respectively. The only relation between the $\widehat{C}_{i 1}$ and $\widehat{C}_{j 2}$ is that their sum is 0 , so that they generate a subspace $V \cong \mathbb{Z}^{2 p-1}$ of $H_{1}(\widehat{S})$. Then, $W$ consists of those $z \in V$ such that $\tau_{*}(z)=-z$.

Since $\tau$ exchanges $\widehat{C}_{i 1}$ and $\widehat{C}_{i 2}$, it follows that $W$ is the abelian subgroup freely generated by the $\widehat{C}_{i 1}-\widehat{C}_{i 2}$, for $i=1, \ldots, p-1$, and by the element $H=\sum_{i=1}^{p} \widehat{C}_{i 1}=$ $-\sum_{i=1}^{p} \widehat{C}_{i 2}$.

As we retract the surface $\widehat{S}$ to the graph $\hat{\Gamma}$, the curves $\widehat{C}_{i 1}$ and $\widehat{C}_{i 2}$ are sent to curves in $\widehat{\Gamma}$ which, because of the alternating condition for the edge orientations at the vertices of $\widehat{\Gamma}$, either follow the orientation of the edges of $\widehat{\Gamma}$ or go against this orientation everywhere. In addition, because $\tau$ reverses the orientation of $\widehat{\Gamma}$, exactly one of these two curves follow the orientation. It follows that, for the identifications $\mathcal{H}(\lambda ; \mathbb{Z}) \cong \mathbb{Z}^{n}$ and $\mathcal{H}(\lambda ; \mathbb{Z}) \cong\left\{\widehat{\alpha} \in H_{1}(\widehat{S} ; \mathbb{Z}) ; \tau_{*}(\widehat{\alpha})=-\widehat{\alpha}\right\}$, the vector $\left(k_{i 1}, k_{i 2}, \ldots, k_{i n}\right)$ corresponds 
to $\varepsilon_{i}\left(\widehat{C}_{i 1}-\widehat{C}_{i 2}\right) \in W \subset H_{1}(\widehat{S} ; \mathbb{Z})$, where $\varepsilon_{i}=+1$ when $C_{i 1}$ is sent to an orientation preserving curve of $\hat{\Gamma}$, and $\varepsilon_{i}=-1$ otherwise. Note that what determines $\varepsilon_{i}$ is our choice of the disk $D \subset \bar{S}$ in the proof of Proposition 5 .

Because each component $\lambda_{i}$ of $\lambda$ has two ends,

$$
(1,1, \ldots, 1)=\frac{1}{2} \sum_{i=1}^{p}\left(k_{i 1}, k_{i 2}, \ldots, k_{i n}\right),
$$

and it follows that $(1,1, \ldots, 1) \in \mathbb{Z}^{n}$ corresponds to

$$
\frac{1}{2} \sum_{i=1}^{p} \varepsilon_{i}\left(\widehat{C}_{i 1}-\widehat{C}_{i 2}\right)=\varepsilon_{p} H+\sum_{i=1}^{p-1} \delta_{i}\left(\widehat{C}_{i 1}-\widehat{C}_{i 2}\right)
$$

with $\delta_{i}=\frac{\varepsilon_{i}-\varepsilon_{p}}{2}= \pm 1$. Since $\operatorname{Ker} \sigma=W$ is freely generated by $H$ and by the $\widehat{C}_{i 1}-\widehat{C}_{i 2}$, for $i=1, \ldots, p-1$, it follows that it is also generated by those elements that, for the identification $\mathcal{H}(\lambda ; \mathbb{Z}) \cong \mathbb{Z}^{n}$, correspond to $(1,1, \ldots, 1)$ and $\left(k_{i 1}, k_{i 2}, \ldots, k_{i n}\right)$, for $i=1, \ldots p-1$.

For a positive integer $N$, we will also need to consider the $N$-kernel of $\sigma$, defined as

$$
\operatorname{Ker}_{N} \sigma=\{\alpha \in \mathcal{H}(\lambda ; \mathbb{Z}) ; \forall \beta \in \mathcal{H}(\lambda ; \mathbb{Z}), \sigma(\alpha, \beta) \in N \mathbb{Z}\}
$$

Note that $\operatorname{Ker}_{N} \sigma$ contains $\mathcal{H}(\lambda ; N \mathbb{Z})$. It therefore makes sense to consider its image in $\mathcal{H}(\lambda ; \mathbb{Z}) / \mathcal{H}(\lambda ; N \mathbb{Z})=\mathcal{H}\left(\lambda ; \mathbb{Z}_{N}\right)$, where $\mathbb{Z}_{N}$ denotes the cyclic group $\mathbb{Z} / N \mathbb{Z}$.

Lemma 9 When $N$ is odd, the $N-$ kernel $\operatorname{Ker}_{N} \sigma$ is equal to the preimage in $\mathcal{H}(\lambda ; \mathbb{Z})$ of the $\mathbb{Z}_{N}$-submodule of $\mathcal{H}\left(\lambda ; \mathbb{Z}_{N}\right) \cong\left(\mathbb{Z}_{N}\right)^{n}$ freely generated by the $p$ vectors $(1,1, \ldots, 1)$ and $\left(k_{i 1}, k_{i 2}, \ldots, k_{i n}\right)$, for $i=1, \ldots p-1$.

Proof The image of the $N$-kernel $\operatorname{Ker}_{N} \sigma$ is the kernel $\operatorname{Ker} \bar{\sigma}$ of the form $\bar{\sigma}$ : $\mathcal{H}\left(\lambda ; \mathbb{Z}_{N}\right) \times \mathcal{H}\left(\lambda ; \mathbb{Z}_{N}\right) \rightarrow \mathbb{Z}_{N}$ induced by $\sigma$. Replacing the coefficient ring $\mathbb{Z}$ by $\mathbb{Z}_{N}$, the proof of Proposition 5 provides an isomorphism $\mathcal{H}\left(\lambda ; \mathbb{Z}_{N}\right) \cong H_{1}\left(\hat{D}_{0} ; \mathbb{Z}_{N}\right) \oplus$ $H_{1}\left(\bar{S} ; \mathbb{Z}_{N}\right) \oplus W_{N}$, where $W_{N}$ is the image of the subspace $W$. The three factors $H_{1}\left(\widehat{D}_{0} ; \mathbb{Z}_{N}\right), H_{1}\left(\bar{S} ; \mathbb{Z}_{N}\right)$ and $W_{N}$ are orthogonal for $\bar{\sigma}$, and the restriction of $\bar{\sigma}$ to each factor is the intersection form of $\widehat{D}_{0}$, twice the intersection form of $\bar{S}$, and 0 , respectively.

Because $N$ is odd, 2 is invertible in $\mathbb{Z}_{N}$. If follows that $\operatorname{Ker} \bar{\sigma}=W_{N}$. The proof of Lemma 8 now shows that $W_{N}$ is freely generated by $(1,1, \ldots, 1)$ and by $\left(k_{i 1}, k_{i 2}, \ldots, k_{i n}\right)$, for $i=1, \ldots b-1$. 
When $N$ is even, $\operatorname{Ker}_{N} \sigma$ contains additional elements. Let $\alpha_{1}, \alpha_{2}, \ldots, \alpha_{2 g}$ form a basis for $H_{1}\left(\bar{S} ; \mathbb{Z}_{2}\right)$. We can represent $\alpha_{i}$ by a family $a_{i}$ of curves immersed in the graph $\Gamma \subset S$ dual to $\lambda$ and passing at most once across each edge of $\Gamma$. Let $l_{i j} \in\{0,1\}$ be the number of times $a_{i}$ traverses the $j$-th edge of $\Gamma$.

Lemma 10 When $N$ is even, the $N$-kernel $\operatorname{Ker}_{N} \sigma$ is equal to the preimage in $\mathcal{H}(\lambda ; \mathbb{Z})$ of the direct sum $A \oplus B \subset \mathcal{H}\left(\lambda ; \mathbb{Z}_{N}\right)$ of the $\mathbb{Z}_{N}$-submodule $A \cong\left(\mathbb{Z}_{N}\right)^{g}$ freely generated by the vectors $(1,1, \ldots, 1)$ and $\left(k_{i 1}, k_{i 2}, \ldots, k_{i n}\right)$ for $i=1, \ldots p-1$, and of the submodule $B \cong\left(\mathbb{Z}_{2}\right)^{2 g}$ generated by the $\left(l_{j 1} \frac{N}{2}, l_{j 2} \frac{N}{2}, \ldots, l_{j n} \frac{N}{2}\right)$ with $j=1, \ldots 2 g$.

Proof The difference with Lemma 10 is that $2 \frac{N}{2}=0$ in $\mathbb{Z}_{N}$. Therefore, in $\mathcal{H}\left(\lambda ; \mathbb{Z}_{N}\right)$ $\cong H_{1}\left(\widehat{D}_{0} ; \mathbb{Z}_{N}\right) \oplus H_{1}\left(\bar{S} ; \mathbb{Z}_{N}\right) \oplus W_{N}$, the kernel Ker $\bar{\sigma}$ is now the direct sum $A \oplus B^{\prime}$ of $A=W_{N}$ and of the subspace $B^{\prime}$ of $H_{1}\left(\bar{S} ; \mathbb{Z}_{N}\right)$ consisting of those elements which are divisible by $\frac{N}{2}$. As before $A=W_{N} \cong\left(\mathbb{Z}_{N}\right)^{g}$ is freely generated by $(1,1, \ldots, 1)$ and by $\left(k_{i 1}, k_{i 2}, \ldots, k_{i n}\right)$, for $i=1, \ldots p-1$.

The factor $B^{\prime}$ is also the image $\frac{N}{2} H_{1}\left(\bar{S} ; \mathbb{Z}_{2}\right)$ of the group homomorphism $H_{1}\left(\bar{S} ; \mathbb{Z}_{2}\right)$ $\rightarrow H_{1}\left(\bar{S} ; \mathbb{Z}_{N}\right)$ defined by multiplication by $\frac{N}{2}$. To identify explicit generators for $B^{\prime} \subset$ $\mathcal{H}\left(\lambda ; \mathbb{Z}_{N}\right)$, it is convenient to consider the transfer map $T: H_{1}\left(S ; \mathbb{Z}_{2}\right) \rightarrow H_{1}\left(\widehat{S} ; \mathbb{Z}_{2}\right)$, which to a cycle in $S$ associates its preimage in $\hat{S}$. Its image is contained in $\{\alpha \in$ $\left.H_{1}\left(\hat{S} ; \mathbb{Z}_{2}\right) ; \tau_{*}(\alpha)=\alpha\right\} \cong \mathcal{H}\left(\lambda ; \mathbb{Z}_{2}\right)$. If $\alpha_{i}^{\prime} \in H_{1}\left(S ; \mathbb{Z}_{2}\right)$ is represented by the above family of curves $a_{i}$, it is immediate from definitions that $T\left(\alpha_{i}^{\prime}\right)$ corresponds to the vector $\left(l_{j 1}, l_{j 2}, \ldots, l_{j n}\right)$ in $\mathcal{H}\left(\lambda ; \mathbb{Z}_{2}\right) \cong\left(\mathbb{Z}_{2}\right)^{n}$.

In the set-up of Proposition 5, the transfer map $T$ can be geometrically realized by representing a class $\alpha \in H_{1}\left(S ; \mathbb{Z}_{2}\right)$ by a curve $a$ contained in $S-D$; then $T(\alpha)$ is the class of $a_{1}+a_{2}$, where $a_{1}$ and $a_{2}$ are copies of $a$ in the two copies $S_{1}$ and $S_{2}$ of $S-D$ contained in $\widehat{S}$. In particular, if we start with a class $\alpha \in H_{1}\left(\bar{S} ; \mathbb{Z}_{2}\right)$, lift it to a class $\alpha^{\prime} \in$ $H_{1}\left(S ; \mathbb{Z}_{2}\right)$ and consider its image $T\left(\alpha^{\prime}\right) \in \mathcal{H}\left(\lambda ; \mathbb{Z}_{2}\right) \cong H_{1}\left(\hat{D}_{0} ; \mathbb{Z}_{2}\right) \oplus H_{1}\left(\bar{S} ; \mathbb{Z}_{2}\right) \oplus W_{2}$, the projection of $T\left(\alpha^{\prime}\right)$ to the factor $H_{1}\left(\bar{S} ; \mathbb{Z}_{2}\right)$ is exactly equal to $\bar{\alpha}$. As a consequence, $H_{1}\left(\bar{S} ; \mathbb{Z}_{2}\right) \oplus W_{2}$ is isomorphic to $B_{2} \oplus W_{2}$ if $B_{2} \subset \mathcal{H}\left(\lambda ; \mathbb{Z}_{2}\right)$ denotes the subspace generated by the $T\left(\alpha_{i}^{\prime}\right)$.

Multiplying everything by $\frac{N}{2}$ we conclude that, in $\mathcal{H}\left(\lambda ; \mathbb{Z}_{N}\right) \cong H_{1}\left(\widehat{D}_{0} ; \mathbb{Z}_{N}\right) \oplus$ $H_{1}\left(\bar{S} ; \mathbb{Z}_{N}\right) \oplus W_{N}$, the kernel $\operatorname{Ker} \bar{\sigma}=0 \oplus \frac{N}{2} H_{1}\left(\bar{S} ; \mathbb{Z}_{2}\right) \oplus W_{N}$ is equal to $0 \oplus B \oplus W_{N}$ where $B=\frac{N}{2} B_{2}$ is generated by the vectors $\left(l_{j 1} \frac{N}{2}, l_{j 2} \frac{N}{2}, \ldots, l_{j n} \frac{N}{2}\right)$. 


\section{The algebraic structure of the Chekhov-Fock algebra}

Lemma 11 The monomials $X_{1}^{k_{1}} X_{2}^{k_{2}} \ldots X_{n}^{k_{n}}$, with $k_{1}, k_{2}, \ldots, k_{n} \in \mathbb{Z}$, form a basis for $\mathcal{T}_{\lambda}^{q}$, considered as a vector space.

Proof This immediately follows from the fact that $\mathcal{T}_{\lambda}^{q}$ is an iterated (Laurent) skewpolynomial algebra, and can also be described as the vector space freely generated by these monomials and endowed with the appropriate multiplication. See Cohn [13, Section 2.1] or Kassel [24, Section 1.7].

Theorem 12 The Chekhov-Fock algebra $\mathcal{T}_{\lambda}^{q}$ is isomorphic to the algebra $\mathcal{W}_{g, k, p}^{q}$ defined by generators $U_{i}^{ \pm 1}, V_{i}^{ \pm 1}$, with $i=1, \ldots, g+k$, and $Z_{j}^{ \pm 1}$ with $j=1, \ldots$, $p$ and by the following relations:

(1) each $U_{i}$ commutes with all generators except $V_{i}^{ \pm 1}$;

(2) each $V_{i}$ commutes with all generators except $U_{i}^{ \pm 1}$;

(3) $U_{i} V_{i}=q^{4} V_{i} U_{i}$ for every $i=1, \ldots, g$;

(4) $U_{i} V_{i}=q^{2} V_{i} U_{i}$ for every $i=g+1, \ldots, g+k$;

(5) each $Z_{j}$ commutes with all generators.

Here $g$ is the genus of the surface $S, p$ is its number of punctures and $k=2 g+p-3$. In addition, the isomorphism between $\mathcal{T}_{\lambda}^{q}$ and $\mathcal{W}_{g, k, p}^{q}$ can be chosen to send monomial to monomial.

Proof Let $F_{n}$ be the free group generated by the set $\left\{X_{1}, \ldots, X_{n}\right\}$. We can rephrase the definition of $\mathcal{T}_{\lambda}^{q}$ by saying that it is is the quotient of the group algebra $\mathbb{C}\left[F_{n}\right]$ by the 2-sided ideal generated by all elements $X_{i} X_{j}-q^{\sigma_{i j}} X_{j} X_{i}$.

Note that the abelianization of $F_{n}$ is canonically isomorphic to $\mathbb{Z}^{n}$. In addition, if we identify two words $a, b \in F_{n}$ to their images in $\mathcal{T}_{\lambda}^{q}$ and if $\bar{a}$ and $\bar{b}$ denote their images in $\mathbb{Z}^{n}$, then $b a=q^{\sigma(\bar{a}, \bar{b})} a b$ in $\mathcal{T}_{\lambda}^{q}$.

Consider the base change isomorphism $\mathbb{Z}^{n} \rightarrow \mathbb{Z}^{n}$ provided by Proposition 5 , under which $\sigma$ becomes block diagonal. Lift this isomorphism to a group isomorphism $F_{n} \rightarrow$ $F_{n}$, which itself induces an algebra isomorphism $\Phi: \mathbb{C}\left[F_{n}\right] \rightarrow \mathbb{C}\left[F_{n}\right]$. If we denote the generators of the first $F_{n}$ by $\left\{U_{1}, V_{1}, U_{2}, V_{2}, \ldots, U_{g+k}, V_{g+k}, Z_{1}, Z_{2}, \ldots, Z_{p}\right\}$, it immediately follows from definitions that $\Phi$ induces an isomorphism from $\mathcal{W}_{g, k, p}^{q}$ to $\mathcal{T}_{\lambda}^{q}$. This isomorphism sends monomial to monomial since it comes from an isomorphism of $F_{n}$. 
The monomials $a X_{1}^{i_{1}} X_{2}^{i_{2}} \ldots X_{n}^{i_{n}}$, with $i_{j} \in \mathbb{Z}$ and $a \in \mathbb{C}$, play a particularly important rôle in the structure of $\mathcal{T}_{\lambda}^{q}$ and of its representations. Let $\mathcal{M}_{\lambda}^{q}$ denote the set of all such monomials that are different from 0 . The multiplication law of $\mathcal{T}_{\lambda}^{q}$ induces a group law on $\mathcal{M}_{\lambda}^{q}$.

The elements $a X_{1}^{0} X_{2}^{0} \ldots X_{n}^{0}$ form a subgroup of $\mathcal{M}_{\lambda}^{q}$ isomorphic to the multiplicative group $\mathbb{C}^{*}=\mathbb{C}-\{0\}$. There is also a natural group homomorphism $\mathcal{M}_{\lambda}^{q} \rightarrow \mathbb{Z}^{n}=\mathcal{H}(\lambda ; \mathbb{Z})$ which to $X=a X_{1}^{i_{1}} X_{2}^{i_{2}} \ldots X_{n}^{i_{n}}$ associates the vector $\bar{X}=\left(i_{1}, i_{2}, \ldots, i_{n}\right)$. This defines a central extension

$$
1 \rightarrow \mathbb{C}^{*} \rightarrow \mathcal{M}_{\lambda}^{q} \rightarrow \mathbb{Z}^{n} \rightarrow 1
$$

whose algebraic structure is completely determined by the commutation property that $X Y=q^{2 \sigma(\bar{X}, \bar{Y})} Y X$ for every $X, Y \in \mathcal{M}_{\lambda}^{q}$.

Let $\mathcal{Z}_{\lambda}^{q}$ be the center of $\mathcal{M}_{\lambda}^{q}$. An immediate consequence of Lemma 11 is that the center of the algebra $\mathcal{T}_{\lambda}^{q}$ consists of all sums of elements of $\mathcal{Z}_{\lambda}^{q}$. We now analyze the structure of $\mathcal{Z}_{\lambda}^{q}$.

We first introduce preferred elements of $\mathcal{Z}_{\lambda}^{q}$. By Lemma $8, \mathcal{Z}_{\lambda}^{q}$ contains the element $X_{1} X_{2} \ldots X_{n}$. However, it is better to introduce its scalar multiple

$$
H=q^{-\sum_{i<i^{\prime}} \sigma_{i i^{\prime}} X_{1} X_{2} \ldots X_{n} .}
$$

Similarly, Lemma 8 shows that the center $\mathcal{Z}_{\lambda}^{q}$ contains the element $X_{1}^{k_{i 1}} X_{2}^{k_{i 2}} \ldots X_{n}^{k_{i n}} \in$ $\mathcal{T}_{\lambda}^{q}$ associated to the $i$-th puncture of $S$, where $k_{i j} \in\{0,1,2\}$ denotes the number of ends of the component $\lambda_{j}$ of $\lambda$ that converge to this $i$-th puncture. Again, we consider

$$
P_{i}=q^{-\sum_{j<j^{\prime}} k_{i j} k_{i j^{\prime}} \sigma_{j j^{\prime}}} X_{1}^{k_{i 1}} X_{2}^{k_{i 2}} \ldots X_{n}^{k_{i n}} .
$$

The $q$-factors in the definition of $H$ and of the $P_{i}$ are specially defined to guarantee invariance under re-indexing of the $X_{j}$. This choice of scalar factors is classically known as the Weyl quantum ordering.

Lemma 13 For every integer $N$ :

$$
\begin{aligned}
& H^{2}=P_{1} P_{2} \ldots P_{p} \\
& H^{N}=q^{-N^{2} \sum_{i<i^{\prime}} \sigma_{i i^{\prime}}} X_{1}^{N} X_{2}^{N} \ldots X_{n}^{N}
\end{aligned}
$$

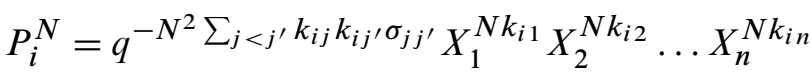

Proof The $P_{i}$ and $H$ belong to the subset $\mathcal{A} \subset \mathcal{Z}_{\lambda}^{q}$ consisting of all elements of the form

$$
q^{-\sum_{j<k} \sigma_{i_{j} i_{k}}} X_{i_{1}} X_{i_{2}} \ldots X_{i_{m}}
$$


Note that the fact that the elements of $\mathcal{A}$ are central implies that $\sum_{k} \sigma_{j i_{k}}=0$ for every $j$. It immediately follows that, for every $A$ and $B \in \mathcal{A}$, the product $A B$ is also in $\mathcal{A}$. Also, an element of $\mathcal{A}$ is invariant under permutation of the $X_{i_{j}}$ (and subsequent adjustment of the $q$-factor).

The three equations of Lemma 13 immediately follow from these observations, using for the first equation the fact that $\sum_{i} k_{i j}=2$ for every $j$.

Proposition 14 When $q$ is not a root of unity, the center $\mathcal{Z}_{\lambda}^{q}$ of the monomial group $\mathcal{M}_{\lambda}^{q}$ is equal to the direct sum of $\mathbb{C}^{*}$ and of the abelian subgroup freely generated (as an abelian group) by the above elements $H$ and $P_{i}$ with $i=1, \ldots, p-1$.

Proof This immediately follows from the algebraic structure of $\mathcal{M}_{\lambda}^{q}$ and from Lemma 8.

When $q^{2}$ is a primitive $N$-th root of unity, the center $\mathcal{Z}_{\lambda}^{q}$ contains additional elements, such as the $X_{i}^{N}$. Lemma 13 provides relations between $H^{N}$, the $X_{i}^{N}$ and the $P_{j}^{N}$.

Proposition 15 If $q^{2}$ is a primitive $N$-th root of unity with $N$ odd, the center $\mathcal{Z}_{\lambda}^{q}$ of the monomial group $\mathcal{M}_{\lambda}^{q}$ is generated by the $X_{i}^{N}$ with $i=1, \ldots, n$, by the element $H$, and by the $P_{j}$ with $j=1, \ldots, p-1$.

In addition, if $W$ denotes the direct sum of $\mathbb{C}^{*}$ and of the free abelian group generated by the $X_{i}^{N}, H$ and $P_{j}$, with $i=1, \ldots, n$ and $j=1, \ldots, p-1$, then $\mathcal{Z}_{\lambda}^{q}$ is isomorphic to the quotient of $W$ by the relations:

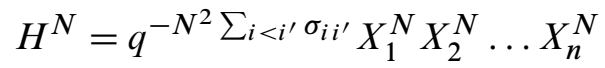

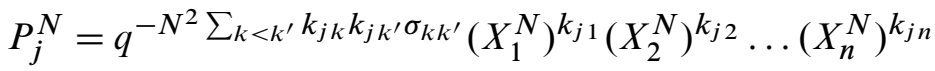

Proof Again, this immediately follows from our analysis of $\operatorname{Ker} \sigma_{N}$ in Lemma 9, together with the relations of Lemma 13.

It should be noted that, when $q^{2}$ is an $N$-th root of unity, then $q^{N}= \pm 1$ so that the $q$-factors in the relations of Proposition 15 are equal to \pm 1 . In later sections, we will choose $q$ so that these factors are actually equal to 1 , making these relations less intimidating.

When $N$ is even, the structure of $\operatorname{Ker} \sigma_{N}$ is more complicated, and consequently so is the structure of $\mathcal{Z}_{\lambda}^{q}$. Let $\alpha_{1}, \alpha_{2}, \ldots, \alpha_{2 g}$ form a basis for $H_{1}\left(\bar{S} ; \mathbb{Z}_{2}\right)$. We can represent $\alpha_{k}$ by a family $a_{k}$ of curves immersed in the graph $\Gamma \subset S$ dual to $\lambda$ and 
passing at most once across each edge of $\Gamma$. Let $l_{k i} \in\{0,1\}$ be the number of times $a_{k}$ traverses the $i$-th edge of $\Gamma$. Define

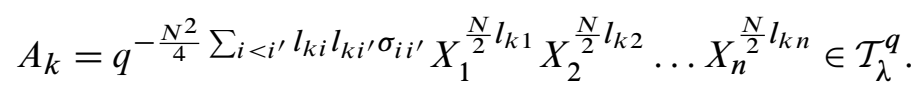

As in Lemma 13,

$$
\begin{aligned}
& A_{k}^{2}=q^{-N^{2} \sum_{i<i^{\prime}} l_{k i} l_{k i^{\prime}} \sigma_{i i^{\prime}}} X_{1}^{N l_{k 1}} X_{2}^{N l_{k 2}} \ldots X_{n}^{N l_{k n}} \\
& =X_{1}^{N l_{k 1}} X_{2}^{N l_{k 2}} \ldots X_{n}^{N l_{k n}}
\end{aligned}
$$

since $q^{N^{2}}=( \pm 1)^{N}=1$ because $N$ is even.

Proposition 16 If $q^{2}$ is a primitive $N$-th root of unity with $N$ even, the center $\mathcal{Z}_{\lambda}^{q}$ of the monomial group $\mathcal{M}_{\lambda}^{q}$ is generated by $\mathbb{C}^{*}$, by the $X_{i}^{N}$ with $i=1, \ldots, n$, by the element $H$, by the $P_{j}$ with $j=1, \ldots, p-1$, and by the $A_{k}$ with $k=1, \ldots, p-1$.

In addition, if $W$ denotes the direct sum of $\mathbb{C}^{*}$ and of the free abelian group generated by the $X_{i}^{N}, H, P_{j}$ and $A_{k}$, with $i=1, \ldots, n, j=1, \ldots, p-1$ and $k=1, \ldots, 2 g$, then $\mathcal{Z}_{\lambda}^{q}$ is isomorphic to the quotient of $W$ by the relations:

$$
\begin{aligned}
H^{N} & =X_{1}^{N} X_{2}^{N} \ldots X_{n}^{N} \\
P_{j}^{N} & =\left(X_{1}^{N}\right)^{k_{j 1}}\left(X_{2}^{N}\right)^{k_{j 2}} \ldots\left(X_{n}^{N}\right)^{k_{j n}} \\
A_{k}^{2} & =\left(X_{1}^{N}\right)^{l_{k 1}}\left(X_{2}^{N}\right)^{l_{k 2}} \ldots\left(X_{n}^{N}\right)^{l_{k n}}
\end{aligned}
$$

Proof Again, this follows from Lemma 10, together with the relations of Lemma 13 and the fact that $q^{N^{2}}=1$ when $N$ is even.

\section{Finite-dimensional representations of the Chekhov-Fock algebra}

This section is devoted to the classification of the finite-dimensional representations of the algebra $\mathcal{T}_{\lambda}^{q}$, namely of the algebra homomorphisms $\rho: \mathcal{T}_{\lambda}^{q} \rightarrow \operatorname{End}(V)$ from $\mathcal{T}_{\lambda}^{q}$ to the algebra of endomorphisms of a finite-dimensional vector space $V$ over $\mathbb{C}$. Recall that two such representations $\rho: \mathcal{T}_{\lambda}^{q} \rightarrow \operatorname{End}(V)$ and $\rho^{\prime}: \mathcal{T}_{\lambda}^{q} \rightarrow \operatorname{End}\left(V^{\prime}\right)$ are isomorphic if there exists a linear isomorphism $L: V \rightarrow V^{\prime}$ such that $\rho^{\prime}(X)=L \cdot \rho(X) \cdot L^{-1}$ for every $X \in \mathcal{T}_{\lambda}^{q}$, where · denotes the composition of maps $V^{\prime} \rightarrow V \rightarrow V \rightarrow V^{\prime}$. Also, $\rho: \mathcal{T}_{\lambda}^{q} \rightarrow \operatorname{End}(V)$ is irreducible if it does not respect any proper subspace $W \subset V$.

Having determined the algebraic structure of $\mathcal{T}_{\lambda}^{q}$ in Section 3, the classification of its representations is an easy exercise (see Lemmas 17, 18 and 19). The main challenge is 
to state this classification in an intrinsic way which is tied to the topology of the ideal triangulation $\lambda$. This is done in Theorem 20 in a first step, and then in Theorems 21 and 22 in a more concrete way.

It is not hard to see that the Chekhov-Fock algebra $\mathcal{T}_{\lambda}^{q}$ cannot admit any finitedimensional representation unless $q$ is a root of unity. In this case, our results will heavily depend on the number $N$ such that $q^{2}$ is a primitive $N$-th root of unity.

In addition to the structure theorems of Section 3, our analysis of the representations of $\mathcal{T}_{\lambda}^{q}$ is based on the following elementary (and classical) facts.

Lemma 17 Let $\mathcal{W}^{q}$ be the algebra defined by the generators $U^{ \pm 1}, V^{ \pm 1}$ and by the relation $U V=q^{2} V U$. If $q^{2}$ is a primitive $N$-th root of unity, every irreducible representation of $\mathcal{W}^{q}$ has dimension $N$, and is isomorphic to a representation $\rho_{u v}$ defined by

$$
\rho_{u v}(U)=u\left(\begin{array}{cccccc}
1 & 0 & 0 & \ldots & 0 & 0 \\
0 & q^{2} & 0 & \ldots & 0 & 0 \\
0 & 0 & q^{4} & \ldots & 0 & 0 \\
\ldots & \ldots & \ldots & \ldots & \ldots & \ldots \\
\ldots & \ldots & \ldots & \ldots & \ldots & \ldots \\
0 & 0 & 0 & \ldots & q^{2 N-4} & 0 \\
0 & 0 & 0 & \ldots & 0 & q^{2 N-2}
\end{array}\right)
$$

and

$$
\rho_{u v}(V)=v\left(\begin{array}{cccccc}
0 & 0 & 0 & \ldots & 0 & 1 \\
1 & 0 & 0 & \ldots & 0 & 0 \\
0 & 1 & 0 & \ldots & 0 & 0 \\
\ldots & \ldots & \ldots & \ldots & \ldots & \ldots \\
\ldots & \ldots & \ldots & \ldots & \ldots & \ldots \\
0 & 0 & 0 & \ldots & 0 & 0 \\
0 & 0 & 0 & \ldots & 1 & 0
\end{array}\right)
$$

for some $u, v \in \mathbb{C}-\{0\}$. In addition, two such representations $\rho_{u v}$ and $\rho_{u^{\prime} v^{\prime}}$ are isomorphic if and only if $u^{N}=\left(u^{\prime}\right)^{N}$ and $v^{N}=\left(v^{\prime}\right)^{N}$.

Proof Note that $U^{N}$ and $V^{N}$ are central in $\mathcal{W}^{q}$. If $\rho$ is an irreducible representation, it must consequently send $U^{N}$ to a homothety $u_{1} \operatorname{Id}$ and $V^{N}$ to a homothety $v_{1}$ Id. In addition, $\rho(V)$ sends an eigenvector of $\rho(U)$ corresponding to an eigenvalue $\ell$ to another eigenvector of $\rho(U)$ corresponding to the eigenvalue $\ell q^{2}$. It easily follows that $\rho$ is isomorphic to a representation $\rho_{u v}$ for some $u, v$ such that $u^{N}=u_{1}$ and $v^{N}=v_{1}$. 
If the representations $\rho_{u v}$ and $\rho_{u^{\prime} v^{\prime}}$ are isomorphic, then necessarily $u^{N}=\left(u^{\prime}\right)^{N}$ and $v^{N}=\left(v^{\prime}\right)^{N}$ by consideration of the homotheties $\rho_{u v}\left(U^{N}\right), \rho_{u^{\prime} v^{\prime}}\left(U^{N}\right), \rho_{u v}\left(V^{N}\right)$ and $\rho_{u^{\prime} v^{\prime}}\left(V^{N}\right)$. Conversely, conjugating $\rho_{u v}$ by the isomorphism $\rho_{u v}(U)$ gives the representation $\rho_{u^{\prime} v^{\prime}}$ with $u^{\prime}=u$ and $v^{\prime}=v q^{2}$; it follows that the isomorphism class of $\rho_{u v}$ depends only on $u$ and $v^{N}$. Similarly, the representation obtained by conjugating $\rho_{u v}$ by the isomorphism $\rho_{u v}(V)$ is equal to the representation $\rho_{u^{\prime} v^{\prime}}$ with $u^{\prime}=u q^{2}$ and $v^{\prime}=v$. It follows that the isomorphism class of $\rho_{u v}$ depends only on $u^{N}$ and $v^{N}$.

Lemma 18 Let $q^{2}$ be a primitive $N$-th root of unity, and let $\mathcal{W}^{q}$ be the algebra defined by the generators $U^{ \pm 1}, V^{ \pm 1}$ and by the relation $U V=q^{2} V U$. Let $\mathcal{W}$ be any algebra. Any irreducible finite-dimensional representation of the tensor product $\mathcal{W} \otimes \mathcal{W}^{q}$ is isomorphic to the tensor product $\rho_{1} \otimes \rho_{2}: \mathcal{W} \otimes \mathcal{W}^{q} \rightarrow \operatorname{End}\left(W_{1} \otimes W_{2}\right)$ of two irreducible representations $\rho_{1}: \mathcal{W} \rightarrow \operatorname{End}\left(W_{1}\right)$ and $\rho_{2}: \mathcal{W}^{q} \rightarrow \operatorname{End}\left(W_{2}\right)$. Conversely, the tensor product of two such irreducible representations is irreducible.

Proof Consider an irreducible representation $\rho: \mathcal{W} \otimes \mathcal{W}^{q} \rightarrow \operatorname{End}(W)$, with $W$ a finite-dimensional vector space over $\mathbb{C}$. Let $W_{1} \subset W$ be an eigenspace of $\rho(1 \otimes U)$, corresponding to the eigenvalue $u$. Then $\rho\left(1 \otimes V^{i}\right)$ sends $W_{1}$ to the eigenspace $W_{i+1}$ of $\rho(1 \otimes U)$ corresponding to the eigenvalue $u q^{2 i}$. Also, $\mathcal{W} \otimes 1$ commutes with $1 \otimes U$, and $\rho(\mathcal{W} \otimes 1)$ consequently preserves each $W_{i}$. Noting that $\rho\left(1 \otimes V^{N}\right)$ is a homothety since $1 \otimes V^{N}$ is central, it follows that $\bigoplus_{i=1}^{N} W_{i}$ is invariant under $\rho\left(\mathcal{W} \otimes \mathcal{W}^{q}\right)$, and is therefore equal to $W$ by irreducibility of $\rho$.

If $\rho(\mathcal{W} \otimes 1)$ respected a proper subspace $W_{1}^{\prime}$ of $W_{1}$, then by the above remarks the subspace $\bigoplus_{i=1}^{N} \rho\left(1 \otimes V^{i}\right)\left(W_{1}^{\prime}\right)$ would be a proper subspace invariant under $\rho(\mathcal{W} \otimes$ $\left.\mathcal{W}^{q}\right)$. By irreducibility of $\rho$, it follows that the representation $\rho_{1}: \mathcal{W} \rightarrow \operatorname{End}\left(W_{1}\right)$ defined by restriction of $\rho(\mathcal{W} \otimes 1)$ to $W_{1}$ is irreducible.

All the pieces are now here to conclude that the representation $\rho$ of $\mathcal{W} \otimes \mathcal{W}^{q}$ over $W=\bigoplus_{i=1}^{N} W_{i}$ is isomorphic to the tensor product of $\rho_{1}: \mathcal{W} \rightarrow \operatorname{End}\left(W_{1}\right)$ and of a representation $\rho_{2}: \mathcal{W}^{q} \rightarrow \operatorname{End}\left(W_{2}\right)$ of the type described in Lemma 17.

Conversely, consider the tensor product $\rho$ of two irreducible representations $\rho_{1}: \mathcal{W} \rightarrow$ $\operatorname{End}\left(W_{1}\right)$ and $\rho_{2}: \mathcal{W}^{q} \rightarrow \operatorname{End}\left(W_{2}\right)$, where $\rho_{2}$ is as in Lemma 17. Let $L_{u} \subset W_{2}$ be the (1-dimensional) eigenspace of $\rho_{2}(U)$ corresponding to the eigenvalue $u$, so that $W_{1} \otimes L_{u}$ is the eigenspace of $\rho(1 \otimes U)$ corresponding to the eigenvalue $u$. If $W^{\prime} \subset W_{1} \otimes W_{2}$ is invariant under $\rho$, in particular it is invariant under $\rho\left(1 \otimes \mathcal{W}^{q}\right)$, and it follows from Lemma 17 that $W^{\prime} \cap\left(W_{1} \otimes L_{u}\right)$ is non-trivial since $\rho\left(1 \otimes U^{N}\right)=u^{N}$ Id. The subspace $W^{\prime} \cap\left(W_{1} \otimes L_{u}\right)$ is also invariant under $\rho(\mathcal{W} \otimes 1)$, and must therefore 
be equal to all of $W_{1} \otimes L_{u}$ by irreducibility of $\rho_{1}$. Therefore, $W^{\prime}$ contains $W_{1} \otimes L_{u}$, from which it easily follows that $W^{\prime}=W_{1} \otimes W_{2}$. This proves that $\rho$ is irreducible.

Lemma 19 Let $\mathbb{C}\left[Z^{ \pm 1}\right]$ be the algebra of Laurent polynomials in the variable $Z$, and let $\mathcal{W}$ be any algebra. Any irreducible finite-dimensional representation of the tensor product $\mathcal{W} \otimes \mathbb{C}\left[Z^{ \pm 1}\right]$ is isomorphic to the tensor product $\rho_{1} \otimes \rho_{2}: \mathcal{W} \otimes$ $\mathbb{C}\left[Z^{ \pm 1}\right] \rightarrow \operatorname{End}\left(V_{1} \otimes V_{2}\right)$ of two irreducible representations $\rho_{1}: \mathcal{W} \rightarrow \operatorname{End}\left(W_{1}\right)$ and $\rho_{2}: \mathbb{C}\left[Z^{ \pm 1}\right] \rightarrow \operatorname{End}\left(W_{2}\right)$. Conversely, the tensor product of two such irreducible representations is irreducible.

Proof This immediately follows from the fact that $Z$ is central in $\mathcal{W} \otimes \mathbb{C}\left[Z^{ \pm 1}\right]$, and from the fact that every irreducible representation $\rho_{2}: \mathbb{C}\left[Z^{ \pm 1}\right] \rightarrow \operatorname{End}\left(W_{2}\right)$ has dimension 1 and is classified by the number $z \in \mathbb{C}^{*}$ such that $\rho_{2}(Z)=z \operatorname{Id}_{W_{2}}$.

Recall that $\mathcal{Z}_{\lambda}^{q}$ denotes the center of the group $\mathcal{M}_{\lambda}^{q}$ of non-zero monomials in the Chekhov-Fock algebra $\mathcal{T}_{\lambda}^{q}$.

Let $\rho: \mathcal{T}_{\lambda}^{q} \rightarrow \operatorname{End}(V)$ be a finite-dimensional irreducible representation of $\mathcal{T}_{\lambda}^{q}$. Every $X \in \mathcal{Z}_{\lambda}^{q}$ is central in $\mathcal{T}_{\lambda}^{q}$, and its image $\rho(X)$ consequently is a homothety, namely of the form $a \operatorname{Id}_{V}$ for $a \in \mathbb{C}$. We can therefore interpret the restriction of $\rho$ to $\mathcal{Z}_{\lambda}^{q} \subset \mathcal{T}_{\lambda}^{q}$ as a group homomorphism $\rho: \mathcal{Z}_{\lambda}^{q} \rightarrow \mathbb{C}^{*}$. Note that $\rho: \mathcal{Z}_{\lambda}^{q} \rightarrow \mathbb{C}^{*}$ coincides with the identity on $\mathbb{C}^{*} \subset \mathcal{Z}_{\lambda}^{q}$.

Theorem 20 Suppose that $q^{2}$ is a primitive $N$-th root of unity. Every irreducible finite-dimensional representation $\rho: \mathcal{T}_{\lambda}^{q} \rightarrow \operatorname{End}(V)$ has dimension $N^{3 g+p-3}$ if $N$ is odd, and $N^{3 g+p-3} / 2^{g}$ if $N$ is even (where $g$ is the genus of the surface $S$ and $p$ is its number of punctures). Up to isomorphism, $\rho$ is completely determined by its restriction $\rho: \mathcal{Z}_{\lambda}^{q} \rightarrow \mathbb{C}^{*}$ to the center $\mathcal{Z}_{\lambda}^{q}$ of the monomial group $\mathcal{M}_{\lambda}^{q}$ of $\mathcal{T}_{\lambda}^{q}$.

Conversely, every group homomorphism $\rho: \mathcal{Z}_{\lambda}^{q} \rightarrow \mathbb{C}^{*}$ coinciding with the identity on $\mathbb{C}^{*} \subset \mathcal{Z}_{\lambda}^{q}$ can be extended to an irreducible finite-dimensional representation $\rho: \mathcal{T}_{\lambda}^{q} \rightarrow$ $\operatorname{End}(V)$.

Proof By Theorem 12 and for $k=2 g+p-3$, the Chekhov-Fock algebra $\mathcal{T}_{\lambda}^{q}$ is isomorphic to the algebra $\mathcal{W}_{g, k, p}^{q}$ defined by generators $U_{i}^{ \pm 1}, V_{i}^{ \pm 1}$, with $i=1, \ldots$, $g+k$, and $Z_{j}^{ \pm 1}$ with $j=1, \ldots, p$ and by the following relations:

(1) each $U_{i}$ commutes with all generators except $V_{i}^{ \pm 1}$;

(2) each $V_{i}$ commutes with all generators except $U_{i}^{ \pm 1}$;

(3) $U_{i} V_{i}=q^{4} V_{i} U_{i}$ for every $i=1, \ldots, g$; 
(4) $U_{i} V_{i}=q^{2} V_{i} U_{i}$ for every $i=g+1, \ldots, g+k$;

(5) each $Z_{j}$ commutes with all generators.

In particular, $\mathcal{T}_{\lambda}^{q}$ is isomorphic to the tensor product of $g$ copies of the algebra $\mathcal{W}^{q^{2}}$ (defined by the generators $U^{ \pm 1}, V^{ \pm 1}$ and by the relation $U V=q^{4} V U$ ), $k$ copies of the algebra $\mathcal{W}^{q}$, and $p$ copies of the algebra $\mathbb{C}\left[Z^{ \pm 1}\right]$. In addition, the isomorphism $\mathcal{W}_{g, k, p}^{q} \cong \mathcal{T}_{\lambda}^{q}$ can be chosen to send the monomial group of $\mathcal{W}_{g, k, p}^{q}$ to the monomial group $\mathcal{M}_{\lambda}^{q}$ of $\mathcal{T}_{\lambda}^{q}$.

By Lemmas 18 and 19, an irreducible finite-dimensional representation is therefore isomorphic to a tensor product $\rho_{1} \otimes \rho_{2} \otimes \cdots \otimes \rho_{g+k+p}$ of irreducible representations $\rho_{i}$ such that $\rho_{i}$ is a representation of $\mathcal{W}^{q}$ for $1 \leqslant i \leqslant g$, a representation of $\mathcal{W}^{q}$ if $g+1 \leqslant i \leqslant g+k$, and a representation of $\mathbb{C}[Z]$ if $g+k+1 \leqslant i \leqslant g+k+p$. In particular, for $g+k+1 \leqslant i \leqslant g+k+p$, the irreducible representation $\rho_{i}$ must have dimension 1 , and is determined by the complex number $\rho_{i}(Z) \in \mathbb{C}^{*}$.

If $N$ is odd, then $q^{2}$ and $q^{4}$ are both primitive $N$-th roots of unity. It follows from Lemma 17 that, for $1 \leqslant i \leqslant g+k$, the representation $\rho_{i}$ has dimension $N$ and is completely determined by the two homotheties $\rho_{i}\left(U_{i}^{N}\right)$ and $\rho_{i}\left(V_{i}^{N}\right)$. As a consequence $\rho$ has dimension $N^{g+k}=N^{3 g+p-3}$, as announced, and is completely determined by the homotheties that are the images of $U_{i}^{N}, V_{j}^{N}$ and $Z_{l}$. Since $U_{i}^{N}$, $V_{j}^{N}$ and $Z_{l}$ belong to the center of the monomial group of $\mathcal{W}_{g, k, p}^{q} \cong \mathcal{T}_{\lambda}^{q}$, this shows that $\rho$ is determined by the restriction of $\rho$ to this center $\mathcal{Z}_{\lambda}^{q}$.

When $N$ is even, then $q^{2}$ is a primitive $N$-th root of unity, but $q^{4}$ is a primitive $\frac{N}{2}$-th root of unity. Lemma 17 now implies that $\rho_{i}$ has dimension $\frac{N}{2}$ if $i=1,2, \ldots, g$, and has dimension $N$ if $g+1 \leqslant i \leqslant g+k$. It follows that $\rho$ has dimension $\left(\frac{N}{2}\right)^{g} N^{k}=$ $N^{3 g+p-3} / 2^{g}$, as announced. In addition, $\rho_{i}$ is determined by the homotheties $\rho\left(U_{i}^{\frac{N}{2}}\right)$ and $\rho\left(V_{i}^{\frac{N}{2}}\right)$ if $i=1,2, \ldots, g$, and by $\rho\left(U_{i}^{N}\right)$ and $\rho\left(V_{i}^{N}\right)$ if $g+1 \leqslant i \leqslant g+k$. Consequently, $\rho$ is completely determined by the images of the $U_{i}^{\frac{N}{2}}, V_{i}^{\frac{N}{2}}$ with $1 \leqslant i \leqslant g$, of the $U_{i}^{N}$ and $V_{i}^{N}$ with $g+1 \leqslant i \leqslant g+k$, and of the $Z_{i}$ with $g+k+1 \leqslant i \leqslant g+k+p$. Since these elements all belong to the center of the monomial group of $\mathcal{W}_{g, k, p}^{q} \cong \mathcal{T}_{\lambda}^{q}$, this shows that $\rho$ is determined by the restriction of $\rho$ to this center $\mathcal{Z}_{\lambda}^{q}$.

This concludes the proof of the first statement of Theorem 20.

We prove the second statement when $N$ is even. The odd case is similar. 
Consider a group homomorphism $\rho: \mathcal{Z}_{\lambda}^{q} \rightarrow \mathbb{C}^{*}$ coinciding with the identity on $\mathbb{C}^{*}$. Lemma 17 associates an irreducible representation $\rho_{i}$ of $\mathcal{W}^{q^{2}}$ to the numbers $\rho\left(U_{i}^{\frac{N}{2}}\right)$ and $\rho\left(V_{i}^{\frac{N}{2}}\right)$ when $1 \leqslant i \leqslant g$, an irreducible representation $\rho_{i}$ of $\mathcal{W}^{q}$ to $\rho\left(U_{i}^{N}\right)$ and $\rho\left(V_{i}^{N}\right)$ when $g+1 \leqslant i \leqslant g+k$. When $g+k+1 \leqslant i \leqslant g+k+p$, there is a 1-dimensional representation $\rho_{i}$ of $\mathbb{C}\left[Z^{ \pm 1}\right]$ such that $\rho_{i}(Z)=\rho\left(Z_{i}\right)$. This defines a representation $\rho^{\prime}=\rho_{1} \otimes \rho_{2} \otimes \cdots \otimes \rho_{g+k+p}$ of $\mathcal{W}_{g, k, p}^{q} \cong \mathcal{T}_{\lambda}^{q}$, which is irreducible by Lemma 18. It remains to show that the group homomorphism $\rho^{\prime}: \mathcal{Z}_{\lambda}^{q} \rightarrow \mathbb{C}^{*}$ induced by $\rho^{\prime}$ coincides with the original group homomorphism $\rho: \mathcal{Z}_{\lambda}^{q} \rightarrow \mathbb{C}^{*}$. But this immediately follows from the fact that the center of the monomial group of $\mathcal{W}_{g, k, p}^{q} \cong \mathcal{T}_{\lambda}^{q}$ is the product of $\mathbb{C}^{*}$ and of the free abelian group generated by the $U_{i}^{\frac{N}{2}}$, $V_{i}^{\frac{N}{2}}$ with $1 \leqslant i \leqslant g$, by the $U_{i}^{N}$ and $V_{i}^{N}$ with $g+1 \leqslant i \leqslant g+k$, and by the $Z_{i}$ with $g+k+1 \leqslant i \leqslant g+k+p$.

This concludes the proof, when $N$ is even, of the property that every $\rho: \mathcal{Z}_{\lambda}^{q} \rightarrow \mathbb{C}^{*}$ coinciding with the identity on $\mathbb{C}^{*}$ can be extended to an irreducible representation $\rho=\rho^{\prime}$ of $\mathcal{T}_{\lambda}^{q}$. As indicated above, the case where $N$ is odd is almost identical.

To express Theorem 20 in a more concrete and geometric way, we now combine this result with our analysis of the algebraic structure of the center $\mathcal{Z}_{\lambda}^{q}$ in Propositions 15 and 16.

Recall that we associated the element

$$
P_{i}=q^{-\sum_{j<j^{\prime}} k_{i j} k_{i j^{\prime}} \sigma_{j j^{\prime}}} X_{1}^{k_{i 1}} X_{2}^{k_{i 2}} \ldots X_{n}^{k_{i n}} \in \mathcal{T}_{\lambda}^{q}
$$

to the $i$-th puncture of $S$, where $k_{i j} \in\{0,1,2\}$ is the number of ends of the component $\lambda_{j}$ of $\lambda$ that converge to this $i$-th puncture. We also considered the element

$$
H=q^{-\sum_{i<i^{\prime}} \sigma_{i i^{\prime}} X_{1} X_{2} \ldots X_{n} .}
$$

Theorem 21 If $q^{2}$ is a primitive $N$-th root of unity with $N$ odd, the irreducible finite-dimensional representation $\rho: \mathcal{T}_{\lambda}^{q} \rightarrow \operatorname{End}(V)$ is, up to isomorphism, completely determined by:

(1) for $i=1,2, \ldots, n$, the number $x_{i} \in \mathbb{C}^{*}$ such that $\rho\left(X_{i}^{N}\right)=x_{i} \operatorname{Id}_{V}$;

(2) for $j=1,2, \ldots, p-1$, the $N$-th root $p_{j}$ of $\varepsilon_{j} x_{1}^{k_{j 1}} x_{2}^{k_{j 2}} \ldots x_{n}^{k_{j n}}$ such that $\rho\left(P_{j}\right)=p_{j} \operatorname{Id}_{V}$

(3) the $N$-th root $h$ of $\varepsilon_{0} x_{1} x_{2} \ldots x_{n}$ such that $\rho(H)=h \operatorname{Id}_{V}$; 


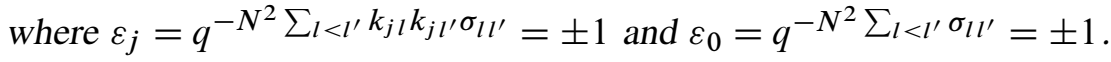

Conversely, every such data of numbers $x_{i}, p_{j}$ and $h \in \mathbb{C}^{*}$ with

$$
p_{j}^{N}=\varepsilon_{j} x_{1}^{k_{j 1}} x_{2}^{k_{j 2}} \ldots x_{n}^{k_{j n}} \text { and } h^{N}=\varepsilon_{0} x_{1} x_{2} \ldots x_{n}
$$

can be realized by an irreducible finite-dimensional representation $\rho: \mathcal{T}_{\lambda}^{q} \rightarrow \operatorname{End}(V)$.

Proof Combine Theorem 20 and Proposition 15.

In the case where $N$ is even, we had to use a basis $\alpha_{1}, \alpha_{2}, \ldots, \alpha_{2 g}$ for $H_{1}\left(\bar{S} ; \mathbb{Z}_{2}\right)$. After representing each $\alpha_{k}$ by a family $a_{k}$ of curves immersed in the graph $\Gamma \subset S$ dual to $\lambda$ and passing $l_{k i} \in\{0,1\}$ times across the $i$-th edge of $\Gamma$, we introduced the monomial

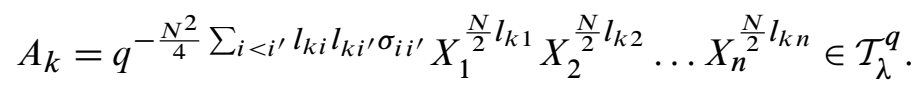

Theorem 22 If $q^{2}$ is a primitive $N$-th root of unity with $N$ even, the irreducible finite-dimensional representation $\rho: \mathcal{T}_{\lambda}^{q} \rightarrow \operatorname{End}(V)$ is, up to isomorphism, completely determined by:

(1) for $i=1,2, \ldots, n$, the number $x_{i} \in \mathbb{C}^{*}$ such that $\rho\left(X_{i}^{N}\right)=x_{i} \operatorname{Id}_{V}$;

(2) for $j=1,2, \ldots, p-1$, the $N$-th root $p_{j}$ of $x_{1}^{k_{j 1}} x_{2}^{k_{j 2}} \ldots x_{n}^{k_{j n}}$ such that $\rho\left(P_{j}\right)=p_{j} \operatorname{Id}_{V}$

(3) the $N$-th root $h$ of $x_{1} x_{2} \ldots x_{n}$ such that $\rho(H)=h \operatorname{Id}_{V}$;

(4) for $k=1,2, \ldots, 2 g$, the square root $a_{k}$ of $x_{1}^{l_{k 1}} x_{2}^{l_{k 2}} \ldots x_{n}^{l_{k n}}$ such that $\rho\left(A_{k}\right)=$ $a_{k} \operatorname{Id}_{V}$.

Conversely, every such data of numbers $x_{i}, p_{j}, h$ and $a_{k} \in \mathbb{C}^{*}$ with

$$
p_{j}^{N}=x_{1}^{k_{j 1}} x_{2}^{k_{j 2}} \ldots x_{n}^{k_{j n}}, \quad h^{N}=x_{1} x_{2} \ldots x_{n} \quad \text { and } a_{k}^{2}=x_{1}^{l_{k 1}} x_{2}^{l_{k 2}} \ldots x_{n}^{l_{k n}}
$$

can be realized by an irreducible finite-dimensional representation $\rho: \mathcal{T}_{\lambda}^{q} \rightarrow \operatorname{End}(V)$.

Proof Combine Theorem 20 and Proposition 16. 


\section{The quantum Teichmüller space}

As one moves from one ideal triangulation $\lambda$ of the surface $S$ to another ideal triangulation $\lambda^{\prime}$, there is a canonical isomorphism $\Phi_{\lambda \lambda^{\prime}}^{q}: \hat{\mathcal{T}}_{\lambda^{\prime}}^{q} \rightarrow \widehat{\mathcal{T}}_{\lambda}^{q}$ between the fraction algebras of the Chekhov-Fock algebras respectively associated to two ideal triangulations $\lambda$ and $\lambda^{\prime}$.

Here the fraction algebra $\hat{\mathcal{T}}_{\lambda}^{q}$ is the division algebra consisting of all the formal fractions $P Q^{-1}$ with $P, Q \in \mathcal{T}_{\lambda}^{q}$ and $Q \neq 0$, subject to the 'obvious' manipulation rules. In other words, $\hat{\mathcal{T}}_{\lambda}^{q}$ is the division algebra of all the non-commutative rational fractions in the variables $X_{i}$, subject to the relations $X_{i} X_{j}=q^{2 \sigma_{i j}} X_{j} X_{i}$. The existence of such a fraction algebra is guaranteed by the fact that $\mathcal{T}_{\lambda}^{q}-\{0\}$ satisfies the so-called Ore condition in $\mathcal{T}_{\lambda}^{q}$; see for instance [13; 24].

The isomorphism $\Phi_{\lambda \lambda^{\prime}}^{q}: \hat{\mathcal{T}}_{\lambda^{\prime}}^{q} \rightarrow \hat{\mathcal{T}}_{\lambda}^{q}$ was introduced by Chekhov and Fock [16] as a quantum deformation of the corresponding change of coordinates in Thurston's shear coordinates for Teichmüller space. See [25] for a version which is more detailed (in particular with respect to non-embedded diagonal exchanges) and is better adapted to the context of the current paper.

To describe the isomorphism $\Phi_{\lambda \lambda^{\prime}}^{q}$, we need to be a little more careful with definitions. We will henceforth agree that the data of an ideal triangulation $\lambda$ also includes an indexing of the components $\lambda_{1}, \lambda_{2}, \ldots, \lambda_{n}$ of $\lambda$ by the set $\{1,2, \ldots, n\}$. Let $\Lambda(S)$ denote the set of isotopy classes of all such (indexed) ideal triangulations of $S$.

The set $\Lambda(S)$ admits two natural operations. The first one is the re-indexing action of the permutation group $\mathfrak{S}_{n}$, which to $\lambda \in \Lambda(S)$ and $\alpha \in \mathfrak{S}_{n}$ associates the indexed ideal triangulation $\alpha \lambda$ whose $i$-th component is equal to $\lambda_{\alpha(i)}$.

The second operation is the $i$-th diagonal exchange $\Delta_{i}: \Lambda(S) \rightarrow \Lambda(S)$ defined as follows. In general, the $i$-th component $\lambda_{i}$ of the ideal triangulation $\lambda$ separates two triangle components $T_{1}$ and $T_{2}$ of $S-\lambda$. The union $T_{1} \cup T_{2} \cup \lambda_{i}$ is an open square $Q$ with diagonal $\lambda_{i}$. Then the ideal triangulation $\Delta_{i}(\lambda) \in \Lambda(S)$ is obtained from $\lambda$ by replacing $\lambda_{i}$ by the other diagonal of the square $Q$, as in Figure 1. This operation is not defined when the two sides of $\lambda_{i}$ are in the same component of $S-\lambda$, which occurs when $\lambda_{i}$ is the only component of $\lambda$ converging to a certain puncture; in this case, we decide that $\Delta_{i}(\lambda)=\lambda$.

It may very well happen that two distinct sides of the square $Q$ correspond to the same component $\lambda_{j}$ of $\lambda$. If, as in Figure 1, we list the components of $\lambda$ in the boundary of $Q$ counterclockwise as $\lambda_{j}, \lambda_{k}, \lambda_{l}$ and $\lambda_{m}$, in such a way that the diagonal $\lambda_{i}$ goes from the $\lambda_{j} \lambda_{k}$ corner to the $\lambda_{l} \lambda_{m}$ corner, we can list all possibilities as follows, up to symmetries of the square: 

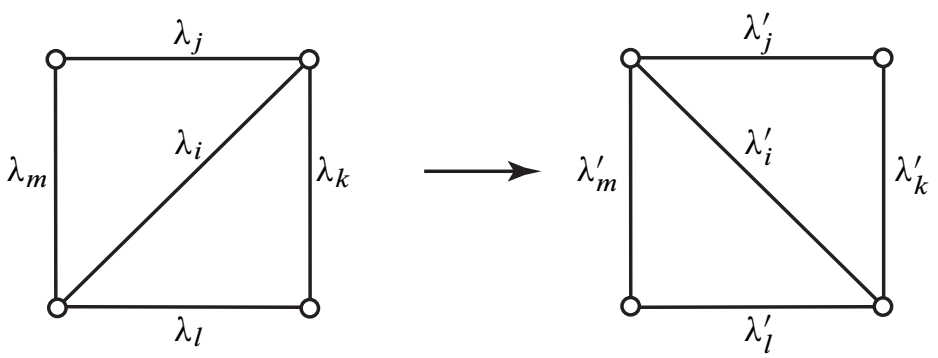

Figure 1

1 The four sides $\lambda_{j}, \lambda_{k}, \lambda_{l}$ and $\lambda_{m}$ of the square $Q$ are all distinct; in this case, we will say that the diagonal exchange is embedded.

$2 \quad \lambda_{j}=\lambda_{l}$ and $\lambda_{k} \neq \lambda_{m}$.

$2^{\prime} \quad \lambda_{k}=\lambda_{m}$ and $\lambda_{j} \neq \lambda_{l}$; note that a diagonal exchange of this type is the inverse of a diagonal exchange of type 2 .

$3 \lambda_{j}=\lambda_{k}$ and $\lambda_{l} \neq \lambda_{m}$.

$\mathbf{3}^{\prime} \quad \lambda_{j}=\lambda_{m}$ and $\lambda_{k} \neq \lambda_{l}$; note that a diagonal exchange of this type is the inverse of a diagonal exchange of type 3 .

$4 \lambda_{j}=\lambda_{l}$ and $\lambda_{k}=\lambda_{m}$; note that $S$ is the once punctured torus in this case.

$5 \quad \lambda_{j}=\lambda_{k}$ and $\lambda_{l}=\lambda_{m}$; note that $S$ is the three-times punctured sphere in this case.

$5^{\prime} \quad \lambda_{j}=\lambda_{m}$ and $\lambda_{k}=\lambda_{l}$; note that a diagonal exchange of this type is the inverse of a diagonal exchange of type 5 .

Observe that these different situations affect the structure of $\mathcal{T}_{\lambda}^{q}$ and $\mathcal{T}_{\lambda^{\prime}}^{q}$ if $\lambda^{\prime}=\Delta_{i}(\lambda)$. For instance, in $\mathcal{T}_{\lambda}^{q}, X_{i} X_{j}$ is equal to $q^{2} X_{j} X_{i}$ in Cases 1 and $2^{\prime}$, is equal to $q^{4} X_{j} X_{i}$ in Cases 2 and 4, and is equal to $X_{j} X_{i}$ in Cases 3, 3', 5 and $5^{\prime}$. Similarly, in $\mathcal{T}_{\lambda^{\prime}}^{q}$, $X_{i} X_{j}$ is equal to $q^{-2} X_{j} X_{i}$ in Cases 1 and $2^{\prime}$, is equal to $q^{-4} X_{j} X_{i}$ in Cases 2 and 4 , and is equal to $X_{j} X_{i}$ in Cases $3,3^{\prime}, 5$ and $5^{\prime}$.

Theorem 23 [16; 25] There is a unique family of isomorphisms $\Phi_{\lambda \lambda^{\prime}}^{q}: \widehat{\mathcal{T}}_{\lambda^{\prime}}^{q} \rightarrow \widehat{\mathcal{T}}_{\lambda}^{q}$, indexed by pairs of ideal triangulations $\lambda, \lambda^{\prime} \in \Lambda(S)$, such that:

(a) for any $\lambda, \lambda^{\prime}$ and $\lambda^{\prime \prime} \in \Lambda(S), \Phi_{\lambda \lambda^{\prime \prime}}^{q}=\Phi_{\lambda \lambda^{\prime}}^{q} \circ \Phi_{\lambda^{\prime} \lambda^{\prime \prime}}^{q}$;

(b) if $\lambda^{\prime}=\alpha \lambda$ is obtained by re-indexing $\lambda \in \Lambda(S)$ by the permutation $\alpha \in \mathfrak{S}_{n}$, $\Phi_{\lambda \lambda^{\prime}}^{q}$ is defined by the property that $\Phi_{\lambda \lambda^{\prime}}^{q}\left(X_{i}\right)=X_{\alpha(i)}$; 
(c) if $\lambda^{\prime}=\Delta_{i}(\lambda)$ is obtained from $\lambda$ by an $i$-th diagonal exchange and if we list all possible configurations as in Cases 1-5' above, then $\Phi_{\lambda \lambda^{\prime}}^{q}$ is defined by the property that $\Phi_{\lambda \lambda^{\prime}}^{q}\left(X_{h}\right)=X_{h}$ for every $h \notin\{i, j, k, l, m\}, \Phi_{\lambda \lambda^{\prime}}^{q}\left(X_{i}\right)=X_{i}^{-1}$, and:

(i) in Case 1,

$$
\begin{array}{ll}
\Phi_{\lambda \lambda^{\prime}}^{q}\left(X_{j}\right)=\left(1+q X_{i}\right) X_{j}, & \Phi_{\lambda \lambda^{\prime}}^{q}\left(X_{k}\right)=\left(1+q X_{i}^{-1}\right)^{-1} X_{k}, \\
\Phi_{\lambda \lambda^{\prime}}^{q}\left(X_{l}\right)=\left(1+q X_{i}\right) X_{l}, & \Phi_{\lambda \lambda^{\prime}}^{q}\left(X_{m}\right)=\left(1+q X_{i}^{-1}\right)^{-1} X_{m} ;
\end{array}
$$

(ii) in Case 2,

$$
\begin{aligned}
& \Phi_{\lambda \lambda^{\prime}}^{q}\left(X_{j}\right)=\left(1+q X_{i}\right)\left(1+q^{3} X_{i}\right) X_{j}, \\
& \Phi_{\lambda \lambda^{\prime}}^{q}\left(X_{k}\right)=\left(1+q X_{i}^{-1}\right)^{-1} X_{k}, \quad \Phi_{\lambda \lambda^{\prime}}^{q}\left(X_{m}\right)=\left(1+q X_{i}^{-1}\right)^{-1} X_{m} ;
\end{aligned}
$$

(iii) in Case 3,

$$
\begin{aligned}
\Phi_{\lambda \lambda^{\prime}}^{q}\left(X_{j}\right) & =X_{i} X_{j}, \quad \Phi_{\lambda \lambda^{\prime}}^{q}\left(X_{l}\right)=\left(1+q X_{i}\right) X_{l}, \\
\Phi_{\lambda \lambda^{\prime}}^{q}\left(X_{m}\right) & =\left(1+q X_{i}^{-1}\right)^{-1} X_{m} ;
\end{aligned}
$$

(iv) in Case 4,

$$
\begin{aligned}
& \Phi_{\lambda \lambda^{\prime}}^{q}\left(X_{j}\right)=\left(1+q X_{i}\right)\left(1+q^{3} X_{i}\right) X_{j}, \\
& \Phi_{\lambda \lambda^{\prime}}^{q}\left(X_{k}\right)=\left(1+q X_{i}^{-1}\right)^{-1}\left(1+q^{3} X_{i}^{-1}\right)^{-1} X_{k} ;
\end{aligned}
$$

(v) in Case 5,

$$
\Phi_{\lambda \lambda^{\prime}}^{q}\left(X_{j}\right)=X_{i} X_{j}, \quad \Phi_{\lambda \lambda^{\prime}}^{q}\left(X_{l}\right)=X_{i} X_{l} .
$$

The uniqueness of $\Phi_{\lambda \lambda^{\prime}}^{q}$ in Theorem 23 immediately comes from the fact that any two ideal triangulations $\lambda$ and $\lambda^{\prime}$ of $S$ can be connected by a finite sequence of diagonal moves and re-indexings (see for instance [32] for this property). The difficult part is to show that the isomorphism $\Phi_{\lambda \lambda^{\prime}}^{q}$ so defined is independent of the choice of the sequence of diagonal moves and re-indexings.

The isomorphisms $\Phi_{\lambda \lambda^{\prime}}^{q}: \widehat{\mathcal{T}}_{\lambda^{\prime}}^{q} \rightarrow \widehat{\mathcal{T}}_{\lambda}^{q}$ enable us to associate an algebraic object to the surface $S$ in a way which does not depend on the choice of an ideal triangulation $\lambda$. For this, consider the set of all pairs $(X, \lambda)$ where $\lambda \in \Lambda(S)$ is an ideal triangulation of $S$ and where $X \in \hat{\mathcal{T}}_{\lambda}^{q}$. Define the quantum Teichmüller space, as

$$
\hat{\mathcal{T}}_{S}^{q}=\left\{(X, \lambda) ; \lambda \in \Lambda(S), X \in \hat{\mathcal{T}}_{\lambda}^{q}\right\} / \sim
$$


where the equivalence relation $\sim$ identifies $(X, \lambda)$ to $\left(X^{\prime}, \lambda^{\prime}\right)$ when $X=\Phi_{\lambda \lambda^{\prime}}^{q}\left(X^{\prime}\right)$. The set $\hat{\mathcal{T}}_{\lambda}^{S}$ inherits a natural division algebra structure from that of the $\hat{\mathcal{T}}_{\lambda}^{q}$. In fact, for any ideal triangulation $\lambda$, there is a natural isomorphism between $\hat{\mathcal{T}}_{S}^{q}$ and $\hat{\mathcal{T}}_{\lambda}^{q}$.

The terminology is motivated by the non-quantum (also called semi-classical) case where $q=1$ (see [16;25], and compare Section 8). Consider the enhanced Teichmüller space $\mathcal{T}(S)$ of $S$, where each element consists of a complete hyperbolic metric defined up to isotopy together with an orientation for each end of $S$ that has infinite area for the metric. Thurston's shear coordinates for Teichmüller space (see for instance [8; 16; 25], [39] for a dual version, and [17] for a generalization) associate to the ideal triangulation $\lambda \in \Lambda(S)$ a diffeomorphism $\varphi_{\lambda}: \mathcal{T}(S) \rightarrow \mathbb{R}^{n}$. The corresponding coordinate changes $\varphi_{\lambda^{\prime}} \circ \varphi_{\lambda}^{-1}: \mathbb{R}^{n} \rightarrow \mathbb{R}^{n}$ are rational functions and, for the natural identifications $\hat{\mathcal{T}}_{\lambda}^{1} \cong \widehat{\mathcal{T}}_{\lambda^{\prime}}^{1} \cong \mathbb{C}\left(X_{1}, X_{2}, \ldots, X_{n}\right)$, it turns out that the isomorphism $\mathbb{C}\left(X_{1}, X_{2}, \ldots, X_{n}\right) \rightarrow \mathbb{C}\left(X_{1}, X_{2}, \ldots, X_{n}\right)$ induced by $\varphi_{\lambda^{\prime}} \circ \varphi_{\lambda}^{-1}$ exactly coincides with $\Phi_{\lambda \lambda^{\prime}}^{1}: \hat{\mathcal{T}}_{\lambda^{\prime}}^{1} \rightarrow \hat{\mathcal{T}}_{\lambda}^{1}$. As a consequence, there is a natural notion of rational functions on $\mathcal{T}(S)$, and the algebra of these rational functions is naturally isomorphic to $\hat{\mathcal{T}}_{S}^{1}$.

For a general $q$, the division algebra $\hat{\mathcal{T}}_{S}^{q}$ can therefore be considered as a deformation of the algebra $\hat{\mathcal{T}}_{S}^{1}$ of all rational functions on the enhanced Teichmüller space $\mathcal{T}(S)$. See [25].

By analogy with the non-quantum situation, we can think of the natural isomorphism $\hat{\mathcal{T}}_{\lambda}^{q} \rightarrow \hat{\mathcal{T}}_{S}^{q}$ as a parametrization of $\hat{\mathcal{T}}_{S}^{q}$ by the explicit algebra $\hat{\mathcal{T}}_{\lambda}^{q}$ associated to the ideal triangulation $\lambda$. Pursuing the analogy, we will call the isomorphism $\Phi_{\lambda \lambda^{\prime}}^{q}: \hat{\mathcal{T}}_{\lambda^{\prime}}^{q} \rightarrow \hat{\mathcal{T}}_{\lambda}^{q}$ the coordinate change isomorphisms associated to the ideal triangulations $\lambda$ and $\lambda^{\prime}$.

Hua Bai [1] proved that the formulas of Theorem 23 are essentially the only ones for which the property holds, once we require the $\Phi_{\lambda \lambda^{\prime}}^{q}$ to satisfy a small number of natural conditions. In particular, the quantum Teichmüller space is a combinatorial object naturally associated to the 2-skeleton of the Harer-Penner simplicial complex [20;32] of ideal cell decompositions of $S$.

For future reference, we note:

Lemma 24 [25] For any two ideal triangulations $\lambda, \lambda^{\prime}$, the coordinate change isomorphism $\Phi_{\lambda \lambda^{\prime}}^{q}: \hat{\mathcal{T}}_{\lambda^{\prime}}^{q} \rightarrow \widehat{\mathcal{T}}_{\lambda}^{q}$ sends the central elements $H, P_{1}, P_{2}, \ldots, P_{p}$ of $\hat{\mathcal{T}}_{\lambda^{\prime}}^{q}$ to the central elements $H, P_{1}, P_{2}, \ldots, P_{p}$ of $\hat{\mathcal{T}}_{\lambda}^{q}$, respectively.

As a consequence, $H$ and the $P_{i}$ give well-defined central elements of the quantum Teichmüller space $\hat{\mathcal{T}}_{S}^{q}$, as well as of its polynomial core $\mathcal{T}_{S}^{q}$ defined in the next section. 


\section{The polynomial core of the quantum Teichmüller space}

The division algebras $\hat{\mathcal{T}}_{\lambda}^{q}$ and $\hat{\mathcal{T}}_{S}^{q}$ have a major drawback. They do not admit any finite-dimensional representations. Indeed, if there was such a finite-dimensional representation $\rho: \hat{\mathcal{T}}_{\lambda}^{q} \rightarrow \operatorname{End}(V)$, then $\rho(Q) \in \operatorname{End}(V)$ would be invertible for every $Q \in \widehat{\mathcal{T}}_{\lambda}^{q}-\{0\}$, by consideration of $\rho\left(Q^{-1}\right)$. However, since $\mathcal{T}_{\lambda}^{q}$ is infinite-dimensional and $\operatorname{End}(V)$ is finite-dimensional, the restriction $\rho: \mathcal{T}_{\lambda}^{q} \rightarrow \operatorname{End}(V)$ has a huge kernel, which provides many $Q$ for which $\rho(Q)=0$ is non-invertible.

On the other hand, we saw in Section 4 that the Chekhov-Fock algebra $\mathcal{T}_{\lambda}^{q}$ admits a rich representation theory. This leads us to introduce the following definition.

Let the polynomial core $\mathcal{T}_{S}^{q}$ of the quantum Teichmüller space $\hat{\mathcal{T}}_{S}^{q}$ be the family $\left\{\mathcal{T}_{\lambda}^{q}\right\}_{\lambda \in \Lambda(S)}$ of all Chekhov-Fock algebras $\mathcal{T}_{\lambda}^{q}$, considered as subalgebras of $\hat{\mathcal{T}}_{S}^{q}$, as $\lambda$ ranges over all ideal triangulations of the surface $S$.

Given two ideal triangulations $\lambda$ and $\lambda^{\prime}$ and two finite-dimensional representations $\rho_{\lambda}: \mathcal{T}_{\lambda}^{q} \rightarrow \operatorname{End}(V)$ and $\rho_{\lambda^{\prime}}: \mathcal{T}_{\lambda^{\prime}}^{q} \rightarrow \operatorname{End}(V)$ of the associated Chekhov-Fock algebras, we would like to say that the two representations correspond to each other under the coordinate change isomorphism $\Phi_{\lambda \lambda^{\prime}}^{q}$, in the sense that $\rho_{\lambda^{\prime}}=\rho_{\lambda} \circ \Phi_{\lambda \lambda^{\prime}}^{q}$. This does not make sense as stated because $\Phi_{\lambda \lambda^{\prime}}^{q}$ is valued in the fraction algebra $\widehat{\mathcal{T}}_{\lambda}^{q}$ and not just in $\mathcal{T}_{\lambda}^{q}$. A natural approach would be, for each $X \in \mathcal{T}_{\lambda^{\prime}}^{q}$, to write the rational fraction $\Phi_{\lambda \lambda^{\prime}}^{q}(X)$ as the quotient $P Q^{-1}$ of two polynomials $P, Q \in \mathcal{T}_{\lambda}^{q}$ and to require that $\rho_{\lambda^{\prime}}(X)=\rho_{\lambda}(P) \rho_{\lambda}(Q)^{-1}$. This of course requires $\rho(Q)$ to be invertible in $\operatorname{End}(V)$, which creates many problems in making the definition consistent. Actually, for a general isomorphism $\Phi: \hat{\mathcal{T}}_{\lambda^{\prime}}^{q} \rightarrow \widehat{\mathcal{T}}_{\lambda}^{q}$ and for a representation $\rho_{\lambda}: \mathcal{T}_{\lambda}^{q} \rightarrow \operatorname{End}(V)$, it is surprisingly difficult to determine under which conditions on $\Phi$ and $\rho_{\lambda}$ they define a representation $\rho_{\lambda} \circ \Phi: \mathcal{T}_{\lambda^{\prime}}^{q} \rightarrow \operatorname{End}(V)$ in the above sense. A lot of these problems can be traced back to the fact that, when adding up fractions $P Q^{-1}$, the usual technique of reduction to a common denominator is much more complicated in the non-commutative context.

We will use an ad hoc definition which strongly uses the definition of $\Phi_{\lambda \lambda^{\prime}}^{q}$. After much work and provided we consider all ideal triangulations at the same time, it will eventually turn out to be equivalent to the above definition.

Given two ideal triangulations $\lambda$ and $\lambda^{\prime}$ and two finite-dimensional representations $\rho_{\lambda}: \mathcal{T}_{\lambda}^{q} \rightarrow \operatorname{End}(V)$ and $\rho_{\lambda^{\prime}}: \mathcal{T}_{\lambda^{\prime}}^{q} \rightarrow \operatorname{End}(V)$ of the associated Chekhov-Fock algebras, we say that $\rho_{\lambda^{\prime}}$ is compatible with $\rho_{\lambda}$ and we write $\rho_{\lambda^{\prime}}=\rho_{\lambda} \circ \Phi_{\lambda \lambda^{\prime}}^{q}$ if, for every generator $X_{i} \in \mathcal{T}_{\lambda^{\prime}}^{q}$, we can write the rational fraction $\Phi_{\lambda \lambda^{\prime}}^{q}\left(X_{i}\right) \in \hat{\mathcal{T}}_{\lambda}^{q}$ as the quotient $P_{i} Q_{i}^{-1}$ of two polynomials $P_{i}, Q_{i} \in \mathcal{T}_{\lambda}^{q}$ in such a way that $\rho_{\lambda}\left(Q_{i}\right)$ is invertible in 
$\operatorname{End}(V)$ and $\rho_{\lambda^{\prime}}\left(X_{i}\right)=\rho_{\lambda}\left(P_{i}\right) \rho_{\lambda}\left(Q_{i}\right)^{-1}$. Note that $\rho_{\lambda}\left(P_{i}\right)$ then is also invertible by consideration of $\rho_{\lambda^{\prime}}\left(X_{i}^{-1}\right)$

At this point, it is not even clear that the relation "is compatible with" is symmetric and transitive. A version of these properties is provided by the following lemma.

Lemma 25 Consider a sequence of ideal triangulations $\lambda_{1}, \lambda_{2}, \ldots, \lambda_{m}$ and finitedimensional representations $\rho_{\lambda_{k}}: \mathcal{T}_{\lambda_{k}}^{q} \rightarrow \operatorname{End}(V)$ such that each $\lambda_{k+1}$ is obtained from $\lambda_{k}$ by a re-indexing or a diagonal exchange. If in addition $\rho_{\lambda_{k}}=\rho_{\lambda_{k+1}} \circ \Phi_{\lambda_{k+1} \lambda_{k}}^{q}$ for every $k$, then $\rho_{\lambda_{1}}=\rho_{\lambda_{m}} \circ \Phi_{\lambda_{m} \lambda_{1}}^{q}$ and $\rho_{\lambda_{m}}=\rho_{\lambda_{1}} \circ \Phi_{\lambda_{1} \lambda_{m}}^{q}$.

Proof We will prove that $\rho_{\lambda_{1}}=\rho_{\lambda_{m}} \circ \Phi_{\lambda_{m} \lambda_{1}}$ by induction on $m$. For this purpose, assume the property true for $m-1$. We need to show that, for every generator $X_{i}$ of $\mathcal{T}_{\lambda_{1}}^{q}, \Phi_{\lambda_{m} \lambda_{1}}\left(X_{i}\right)$ can be written as a quotient $P Q^{-1}$ where $P, Q \in \mathcal{T}_{\lambda_{1}}^{q}$ are such that $\rho_{\lambda_{m}}(P)$ and $\rho_{\lambda_{m}}(Q)$ are invertible and $\rho_{\lambda_{1}}\left(X_{i}\right)=\rho_{\lambda_{m}}(P) \rho_{\lambda_{m}}(Q)^{-1}$.

If $\lambda_{m}$ is obtained from $\lambda_{m-1}$ by re-indexing, then the property immediately follows from the induction hypothesis after re-indexing of the $X_{i}$.

We can therefore restrict attention to the case where $\lambda_{m}$ is obtained from $\lambda_{m-1}$ by one diagonal exchange, along the $i_{0}-$ th component of $\lambda_{m-1}$, say.

The general strategy of the proof is fairly straightforward, but the non-commutative context makes it hard to control which elements have an invertible image under $\rho_{\lambda_{m}}$; this requires more care that one might have anticipated at first glance.

We need to be a little careful in our notation. Let $\mathbb{C}\left\{Z_{1}^{ \pm 1}, Z_{2}^{ \pm 1}, \ldots, Z_{n}^{ \pm 1}\right\}$ denote the algebra of non-commutative polynomials in the $2 n$ variables $Z_{1}, Z_{2}, \ldots, Z_{n}, Z_{1}^{-1}$, $Z_{2}^{-1}, \ldots, Z_{n}^{-1}$. Given such a polynomial $P \in \mathbb{C}\left\{Z_{1}^{ \pm 1}, Z_{2}^{ \pm 1}, \ldots, Z_{n}^{ \pm 1}\right\}$ and invertible elements $A_{1}, A_{2}, \ldots A_{n}$ of an algebra $\mathcal{A}$, we will denote by $P\left(A_{1}, A_{2}, \ldots, A_{n}\right)$ the element of $\mathcal{A}$ defined by replacing each $Z_{i}$ by the corresponding $A_{i}$ and each $Z_{i}^{-1}$ by $A_{i}^{-1}$.

Consider the generator $X_{i} \in \mathcal{T}_{\lambda_{1}}^{q}$. By induction hypothesis,

$$
\Phi_{\lambda_{m-1} \lambda_{1}}^{q}\left(X_{i}\right)=P\left(X_{1}, \ldots, X_{n}\right) Q\left(X_{1}, \ldots, X_{n}\right)^{-1}
$$

in $\hat{\mathcal{T}}_{\lambda_{m-1}^{q}}^{q}$, for some non-commutative polynomials $P$ and $Q$ with

$$
\rho_{\lambda_{m-1}}\left(P\left(X_{1}, X_{2}, \ldots, X_{n}\right)\right) \text { and } \rho_{\lambda_{m-1}}\left(Q\left(X_{1}, X_{2}, \ldots, X_{n}\right)\right)
$$

invertible in $\operatorname{End}(V)$; beware that $X_{i}$ represents a generator of $\mathcal{T}_{\lambda_{1}}^{q}$ in the left hand side of the equation, and a generator of $\mathcal{T}_{\lambda_{m-1}}^{q}$ in the right hand side. In addition,

$$
\rho_{\lambda_{1}}\left(X_{i}\right)=\rho_{\lambda_{m-1}}\left(P\left(X_{1}, X_{2}, \ldots, X_{n}\right)\right) \rho_{\lambda_{m-1}}\left(Q\left(X_{1}, X_{2}, \ldots, X_{n}\right)\right)^{-1} .
$$


Then,

$$
\begin{aligned}
\Phi_{\lambda_{m} \lambda_{1}}^{q}\left(X_{i}\right)= & \Phi_{\lambda_{m} \lambda_{m-1}}^{q} \circ \Phi_{\lambda_{m-1} \lambda_{1}}^{q}\left(X_{i}\right) \\
= & \Phi_{\lambda_{m} \lambda_{m-1}}^{q}\left(P\left(X_{1}, \ldots, X_{n}\right)\right) \Phi_{\lambda_{m} \lambda_{m-1}}^{q}\left(Q\left(X_{1}, \ldots, X_{n}\right)\right)^{-1} \\
= & P\left(\Phi_{\lambda_{m} \lambda_{m-1}}^{q}\left(X_{1}\right), \ldots, \Phi_{\lambda_{m} \lambda_{m-1}}^{q}\left(X_{n}\right)\right) \\
& \quad Q\left(\Phi_{\lambda_{m} \lambda_{m-1}}^{q}\left(X_{1}\right), \ldots, \Phi_{\lambda_{m} \lambda_{m-1}}^{q}\left(X_{n}\right)\right)^{-1} .
\end{aligned}
$$

We are now facing the problem of reducing these quantities to a common denominator, while controlling the invertibility of the images of denominators under $\rho_{\lambda_{m}}$.

The ideal triangulation $\lambda_{m}$ is obtained from $\lambda_{m-1}$ by a diagonal exchange along its $i_{0}$-th component. By inspection in the formulas defining $\Phi_{\lambda_{m} \lambda_{m-1}}$, it follows that $P\left(\Phi_{\lambda_{m} \lambda_{m-1}}^{q}\left(X_{1}\right), \ldots, \Phi_{\lambda_{m} \lambda_{m-1}}^{q}\left(X_{n}\right)\right)$ is a polynomial in the terms $X_{j}^{ \pm 1},(1+$ $\left.q X_{i_{0}}^{ \pm 1}\right)^{-1}$ and possibly $\left(1+q^{3} X_{i_{0}}^{ \pm 1}\right)^{-1}$. In addition, whenever a factor $\left(1+q X_{i_{0}}^{ \pm 1}\right)^{-1}$ or $\left(1+q^{3} X_{i_{0}}^{ \pm 1}\right)^{-1}$ appears, it is through a relation such as

$$
\begin{aligned}
\Phi_{\lambda_{m} \lambda_{m-1}}\left(X_{j}^{-1}\right) & =X_{j}^{-1}\left(1+q X_{i_{0}}\right)^{-1} \\
\text { or } \quad \Phi_{\lambda_{m} \lambda_{m-1}}\left(X_{j}\right) & =\left(1+q X_{i_{0}}^{-1}\right)^{-1}\left(1+q^{3} X_{i_{0}}^{-1}\right)^{-1} X_{j}
\end{aligned}
$$

(there are two more possibilities), which respectively give

$$
\begin{aligned}
& \rho_{\lambda_{m}}\left(1+q X_{i_{0}}\right)=\rho_{\lambda_{m-1}}\left(X_{j}\right) \rho_{\lambda_{m}}\left(X_{j}^{-1}\right), \\
& \rho_{\lambda_{m}}\left(1+q^{3} X_{i_{0}}^{-1}\right) \rho_{\lambda_{m}}\left(1+q X_{i_{0}}^{-1}\right)=\rho_{\lambda_{m}}\left(X_{j}\right) \rho_{\lambda_{m-1}}\left(X_{j}^{-1}\right),
\end{aligned}
$$

or two more relations, using the property that $\rho_{\lambda_{m-1}}=\rho_{\lambda_{m}} \circ \Phi_{\lambda_{m} \lambda_{m-1}}^{q}$. Since $\rho_{\lambda_{m}}\left(X_{j}^{ \pm 1}\right)$ and $\rho_{\lambda_{m-1}}\left(X_{j}^{ \pm 1}\right)$ are invertible and since $V$ is finite-dimensional we conclude that, for every $\left(1+q X_{i_{0}}^{ \pm 1}\right)^{-1}$ or $\left(1+q^{3} X_{i_{0}}^{ \pm 1}\right)^{-1}$ appearing in

$$
P\left(\Phi_{\lambda_{m} \lambda_{m-1}}^{q}\left(X_{1}\right), \ldots, \Phi_{\lambda_{m} \lambda_{m-1}}^{q}\left(X_{n}\right)\right),
$$

the corresponding element $\rho_{\lambda_{m}}\left(1+q X_{i_{0}}^{ \pm 1}\right)$ or $\rho_{\lambda_{m}}\left(1+q^{3} X_{i_{0}}^{ \pm 1}\right)$ is invertible in $\operatorname{End}(V)$.

Now, using the skew-commutativity relations

$$
\left(1+q^{2 k+1} X_{i_{0}}^{ \pm 1}\right) X_{j}=X_{j}\left(1+q^{2 k \pm \sigma_{i_{0} j}+1} X_{i_{0}}^{ \pm 1}\right),
$$

we can push all the $\left(1+q^{2 k+1} X_{i_{0}}^{ \pm 1}\right)^{-1}$ to the right in the expression of

$$
P\left(\Phi_{\lambda_{m} \lambda_{m-1}}^{q}\left(X_{1}\right), \ldots, \Phi_{\lambda_{m} \lambda_{m-1}}^{q}\left(X_{n}\right)\right),
$$


leading to a relation

$$
P\left(\Phi_{\lambda_{m} \lambda_{m-1}}^{q}\left(X_{1}\right), \ldots, \Phi_{\lambda_{m} \lambda_{m-1}}^{q}\left(X_{n}\right)\right)=P^{\prime}\left(X_{1}, \ldots, X_{n}\right) R\left(X_{i_{0}}\right)^{-1}
$$

where $P^{\prime}\left(X_{1}, \ldots, X_{n}\right)$ is a Laurent polynomial in the $X_{j}$ and where $R\left(X_{i_{0}}\right)$ is a 1variable Laurent polynomial product of terms $\left(1+q^{2 k+1} X_{i_{0}}^{ \pm 1}\right)$. In addition, applying $\rho_{\lambda_{m}}$ to both sides of the above skew-commutativity relation, we see that $\rho_{\lambda_{m}}(1+$ $\left.q^{2 k+1} X_{i_{0}}^{ \pm 1}\right)$ is invertible in $\operatorname{End}(V)$ whenever a term $\left(1+q^{2 k+1} X_{i_{0}}^{ \pm 1}\right)^{-1}$ appears in this process. Therefore, $\rho_{\lambda_{m}}\left(R\left(X_{i_{0}}\right)\right)$ is invertible.

We will now perform essentially the same computations in $\operatorname{End}(V)$. Since $\rho_{\lambda_{m-1}}=$ $\rho \lambda_{m} \circ \Phi_{\lambda_{m} \lambda_{m-1}}^{q}$,

$$
\begin{aligned}
\rho_{\lambda_{m-1}}\left(P\left(X_{1}, \ldots, X_{n}\right)\right) & =P\left(\rho_{\lambda_{m-1}}\left(X_{1}\right), \ldots, \rho_{\lambda_{m-1}}\left(X_{n}\right)\right) \\
& =P\left(\rho_{\lambda_{m}} \circ \Phi_{\lambda_{m} \lambda_{m-1}}^{q}\left(X_{1}\right), \ldots, \rho_{\lambda_{m}} \circ \Phi_{\lambda_{m} \lambda_{m-1}}^{q}\left(X_{n}\right)\right)
\end{aligned}
$$

The same manipulations as above, but replacing the $X_{j}$ by the $\rho_{\lambda_{m}}\left(X_{j}\right) \in \operatorname{End}(V)$ (which satisfy the same relations), yield

$$
\begin{aligned}
\rho_{\lambda_{m-1}}\left(P\left(X_{1}, \ldots, X_{n}\right)\right) & =P\left(\rho_{\lambda_{m}} \circ \Phi_{\lambda_{m} \lambda_{m-1}}^{q}\left(X_{1}\right), \ldots, \rho_{\lambda_{m}} \circ \Phi_{\lambda_{m} \lambda_{m-1}}^{q}\left(X_{n}\right)\right) \\
& =P^{\prime}\left(\rho_{\lambda_{m}}\left(X_{1}\right), \ldots, \rho_{\lambda_{m}}\left(X_{n}\right)\right) R\left(\rho_{\lambda_{m}}\left(X_{i_{0}}\right)\right)^{-1} \\
& =\rho_{\lambda_{m}}\left(P^{\prime}\left(X_{1}, \ldots, X_{n}\right)\right) \rho_{\lambda_{m}}\left(R\left(X_{i_{0}}\right)\right)^{-1}
\end{aligned}
$$

In particular, since $\rho_{\lambda_{m-1}}\left(P\left(X_{1}, X_{2}, \ldots, X_{n}\right)\right)$ is invertible by definition of $P$ and $Q$ and since $\rho_{\lambda_{m}}\left(R\left(X_{i_{0}}\right)\right)$ is invertible by construction, we conclude that $\rho_{\lambda_{m}}\left(P^{\prime}\left(X_{1}, \ldots, X_{n}\right)\right)$ is invertible.

Similarly, we can write

$$
Q\left(\Phi_{\lambda_{m} \lambda_{m-1}}^{q}\left(X_{1}\right), \ldots, \Phi_{\lambda_{m} \lambda_{m-1}}^{q}\left(X_{n}\right)\right)=Q^{\prime}\left(X_{1}, \ldots, X_{n}\right) S\left(X_{i_{0}}\right)^{-1}
$$

for some Laurent polynomials $Q^{\prime}\left(X_{1}, \ldots, X_{n}\right)$ and $S\left(X_{i_{0}}\right)$, in such a way that $\rho_{\lambda_{m}}\left(Q_{i}\left(X_{1}, \ldots, X_{n}\right)\right)$ and $\rho_{\lambda_{m}}\left(S\left(X_{i_{0}}\right)\right)$ are invertible in $\operatorname{End}(V)$, and

$$
\rho_{\lambda_{m-1}}\left(Q\left(X_{1}, \ldots, X_{n}\right)\right)=\rho_{\lambda_{m}}\left(Q^{\prime}\left(X_{1}, \ldots, X_{n}\right)\right) \rho_{\lambda_{m}}\left(S\left(X_{i_{0}}\right)\right)^{-1} .
$$

We are now ready to conclude. Indeed, we showed that

$$
\begin{aligned}
\Phi_{\lambda_{m} \lambda_{1}}^{q}\left(X_{i}\right)= & P\left(\Phi_{\lambda_{m} \lambda_{m-1}}^{q}\left(X_{1}\right), \ldots, \Phi_{\lambda_{m} \lambda_{m-1}}^{q}\left(X_{n}\right)\right) \\
& Q\left(\Phi_{\lambda_{m} \lambda_{m-1}}^{q}\left(X_{1}\right), \ldots, \Phi_{\lambda_{m} \lambda_{m-1}}^{q}\left(X_{n}\right)\right)^{-1} \\
= & \left(P^{\prime}\left(X_{1}, \ldots, X_{n}\right) R\left(X_{i_{0}}\right)^{-1}\right)\left(Q^{\prime}\left(X_{1}, \ldots, X_{n}\right) S\left(X_{i_{0}}\right)^{-1}\right)^{-1} \\
= & \left(P^{\prime}\left(X_{1}, \ldots, X_{n}\right) S\left(X_{i_{0}}\right)\right)\left(Q^{\prime}\left(X_{1}, \ldots, X_{n}\right) R\left(X_{i_{0}}\right)\right)^{-1}
\end{aligned}
$$


since $R\left(X_{i_{0}}\right)$ and $S\left(X_{i_{0}}\right)$ commute. Similarly,

$$
\begin{aligned}
\rho_{\lambda_{1}}\left(X_{i}\right)= & \rho_{\lambda_{m-1}}\left(P\left(X_{1}, X_{2}, \ldots, X_{n}\right)\right) \rho_{\lambda_{m-1}}\left(Q\left(X_{1}, X_{2}, \ldots, X_{n}\right)\right)^{-1} \\
= & \left(\rho_{\lambda_{m}}\left(P^{\prime}\left(X_{1}, \ldots, X_{n}\right)\right) \rho_{\lambda_{m}}\left(R\left(X_{i_{0}}\right)\right)^{-1}\right) \\
& \left(\rho_{\lambda_{m}}\left(Q^{\prime}\left(X_{1}, \ldots, X_{n}\right)\right) \rho_{\lambda_{m}}\left(S\left(X_{i_{0}}\right)\right)^{-1}\right)^{-1} \\
= & \left(\rho_{\lambda_{m}}\left(P^{\prime}\left(X_{1}, \ldots, X_{n}\right)\right) \rho_{\lambda_{m}}\left(S\left(X_{i_{0}}\right)\right)\right) \\
& \left(\rho_{\lambda_{m}}\left(Q^{\prime}\left(X_{1}, \ldots, X_{n}\right)\right) \rho_{\lambda_{m}}\left(R\left(X_{i_{0}}\right)\right)\right)^{-1} \\
= & \rho_{\lambda_{m}}\left(P^{\prime}\left(X_{1}, \ldots, X_{n}\right) S\left(X_{i_{0}}\right)\right) \rho_{\lambda_{m}}\left(Q^{\prime}\left(X_{1}, \ldots, X_{n}\right) R\left(X_{i_{0}}\right)\right)^{-1}
\end{aligned}
$$

By definition, this means that $\rho_{\lambda_{1}}=\rho_{\lambda_{m}} \circ \Phi_{\lambda_{m} \lambda_{1}}$, as desired.

There remains to prove the second statement that $\rho_{\lambda_{m}}=\rho_{\lambda_{1}} \circ \Phi_{\lambda_{1} \lambda_{m}}^{q}$. For this, note that the property that $\rho_{\lambda_{k}}=\rho_{\lambda_{k+1}} \circ \Phi_{\lambda_{k+1} \lambda_{k}}^{q}$ implies that $\rho_{\lambda_{k+1}}=\rho_{\lambda_{k}} \circ \Phi_{\lambda_{k} \lambda_{k+1}}^{q}$ for every $k$, using the explicit form of $\Phi_{\lambda_{k+1} \lambda_{k}}^{q}$ and $\Phi_{\lambda_{k} \lambda_{k+1}}^{q}$ as well as arguments which are similar to and much simpler than the ones we just used. The property that $\rho_{\lambda_{m}}=\rho_{\lambda_{1}} \circ \Phi_{\lambda_{1} \lambda_{m}}^{q}$ then immediately follows by symmetry.

A representation of the polynomial core $\mathcal{T}_{S}^{q}$ over the vector space $V$ is a family of representations $\rho_{\lambda}: \mathcal{T}_{\lambda}^{q} \rightarrow \operatorname{End}(V)$ defined for each ideal triangulation $\lambda \in \Lambda(S)$, such that any two $\rho_{\lambda^{\prime}}$ and $\rho_{\lambda}$ are compatible in the above sense. Lemma 25 shows that it suffices to check this condition on pairs of ideal triangulations which are obtained from each other by one re-indexing or one diagonal exchange. We will see in the next sections that the polynomial core admits many representations.

Before closing this section, we indicate the following result, which shows that our definition of compatibility coincides with the condition we had in mind at the beginning of this section.

Lemma 26 Let $\rho=\left\{\rho_{\lambda}: \mathcal{T}_{\lambda}^{q} \rightarrow \operatorname{End}(V)\right\}_{\lambda \in \Lambda(S)}$ be a finite-dimensional irreducible representation of the polynomial core $\mathcal{T}_{S}^{q}$ of the quantum Teichmüller space $\hat{\mathcal{T}}_{S}^{q}$. Then, for every $X^{\prime} \in \mathcal{T}_{\lambda^{\prime}}^{q}$, its image $\Phi_{\lambda \lambda^{\prime}}^{q}\left(X^{\prime}\right) \in \widehat{\mathcal{T}}_{\lambda}^{q}$ can be written as $\Phi_{\lambda \lambda^{\prime}}^{q}\left(X^{\prime}\right)=$ $P Q^{-1}=\left(Q^{\prime}\right)^{-1} P^{\prime}$ with $P, Q \in \mathcal{T}_{\lambda}^{q}$ and with $\rho_{\lambda}(Q)$ and $\rho_{\lambda}\left(Q^{\prime}\right)$ invertible in End $(V)$. In addition, for any such decomposition of $\Phi_{\lambda \lambda^{\prime}}^{q}\left(X^{\prime}\right), \rho_{\lambda^{\prime}}\left(X^{\prime}\right)$ is then equal to $\rho_{\lambda}(P) \rho(Q)^{-1}=\rho\left(Q^{\prime}\right)^{-1} \rho_{\lambda}\left(P^{\prime}\right)$.

Proof This is proved by arguments almost identical to the ones we used for Lemma 25 , by induction on the number of diagonal exchanges needed to go from $\lambda$ to $\lambda^{\prime}$. 
However, it is worth mentioning that the easy algebraic manipulation leading to the last statement simultaneously uses the left and right decompositions $P Q^{-1}$ and $\left(Q^{\prime}\right)^{-1} P^{\prime}$ of $\Phi_{\lambda \lambda^{\prime}}^{q}\left(X^{\prime}\right)$.

\section{The non-quantum shadow of a representation}

By Theorems 21 and 22, an irreducible finite-dimensional representation $\rho_{\lambda}: \mathcal{T}_{\lambda}^{q} \rightarrow$ $\operatorname{End}(V)$ of the Chekhov-Fock algebra is classified, up to a finite number of choices of certain roots, by numbers $x_{i} \in \mathbb{C}^{*}$ associated to the components $\lambda_{i}$ of $\lambda$. By Theorem 21 or by inspection, the same numbers $x_{i}$ completely determine a representation $\rho_{\lambda}^{1}: \mathcal{T}_{\lambda}^{1} \rightarrow \operatorname{End}(\mathbb{C})$ of the commutative algebra $\mathcal{T}_{\lambda}^{1}$ corresponding to the non-quantum (also called semi-classical in the physics literature) case where $q=1$. We will say that $\rho_{\lambda}^{1}$ is the non-quantum shadow, or the semi-classical shadow, of the representation $\rho_{\lambda}$.

Interpreting the numbers $x_{i} \in \mathbb{C}^{*}$ as a non-quantum representation $\rho_{\lambda}^{1}: \mathcal{T}_{\lambda}^{1} \rightarrow \operatorname{End}(\mathbb{C})$ may sound really pedantic at first. However, the remainder of this paper hinges on the following computation which shows that, for a suitable choice of $q$, the map $\rho_{\lambda} \mapsto \rho_{\lambda}^{1}$ is well-behaved with respect to the coordinate changes $\Phi_{\lambda \lambda^{\prime}}^{q}$ and $\Phi_{\lambda \lambda^{\prime}}^{1}$.

Lemma 27 Let $q$ be such that $q^{2}$ is a primitive $N$-th root of unity and such that $q^{N}=(-1)^{N+1}$ (for instance $q=-\mathrm{e}^{\pi \mathrm{i} / N}$ ). Suppose that the two ideal triangulations $\lambda$ and $\lambda^{\prime}$ of the surface $S$ are obtained from each other by a diagonal exchange or by a re-indexing, and consider two irreducible finite-dimensional representations $\rho_{\lambda}: \mathcal{T}_{\lambda}^{q} \rightarrow \operatorname{End}(V)$ and $\rho_{\lambda^{\prime}}: \mathcal{T}_{\lambda^{\prime}}^{q} \rightarrow \operatorname{End}(V)$ such that $\rho_{\lambda^{\prime}}=\rho_{\lambda} \circ \Phi_{\lambda \lambda^{\prime}}^{q}$ in the sense of Section 6. If $\rho_{\lambda}^{1}: \mathcal{T}_{\lambda}^{1} \rightarrow \operatorname{End}(\mathbb{C})$ and $\rho_{\lambda^{\prime}}^{1}: \mathcal{T}_{\lambda^{\prime}}^{1} \rightarrow \operatorname{End}(\mathbb{C})$ are the respective nonquantum shadows of $\rho_{\lambda}$ and $\rho_{\lambda^{\prime}}$, then $\rho_{\lambda^{\prime}}^{1}=\rho_{\lambda}^{1} \circ \Phi_{\lambda \lambda^{\prime}}^{1}$.

Proof Recall that $\rho_{\lambda}^{1}$ is determined by the property that $\rho_{\lambda}^{1}\left(X_{i}\right)=x_{i} \in \mathbb{C}^{*} \subset \operatorname{End}(\mathbb{C})$, where $x_{i}$ is the number such that $\rho_{\lambda}\left(X_{i}^{N}\right)=x_{i} \operatorname{Id}_{V}$. Similarly, $\rho_{\lambda^{\prime}}^{1}\left(X_{i}\right)=x_{i}^{\prime}$ where $x_{i}^{\prime}$ is such that $\rho_{\lambda^{\prime}}\left(X_{i}^{N}\right)=\rho_{\lambda} \circ \Phi_{\lambda \lambda^{\prime}}\left(X_{i}^{N}\right)=x_{i}^{\prime} \operatorname{Id}_{V}$. In particular, the property is immediate when $\lambda^{\prime}$ is obtained from $\lambda$ by a re-indexing of its components.

Suppose that $\lambda^{\prime}$ is obtained from $\lambda$ by an embedded $i$-th diagonal exchange. Label the four sides of the square $Q$ supporting the exchange counterclockwise as $\lambda_{j}, \lambda_{k}$, $\lambda_{l}$ and $\lambda_{m}$, in such a way that the diagonal $\lambda_{i}$ goes from the $\lambda_{j} \lambda_{k}$ corner to the $\lambda_{l} \lambda_{m}$ corner, as in Figure 1.

By definition of $\Phi_{\lambda \lambda^{\prime}}^{q}, \Phi_{\lambda \lambda^{\prime}}^{q}\left(X_{i}^{N}\right)=X_{i}^{-N}$. Using Lemma 26, it follows that $\rho_{\lambda^{\prime}}\left(X_{i}^{N}\right)$ $=\rho_{\lambda}\left(X_{i}^{N}\right)^{-1}$, so that $x_{i}^{\prime}=x_{i}^{-1}$. 
Because $X_{j} X_{i}=q^{2} X_{i} X_{j}$, the quantum binomial formula (see for instance [24, Section IV.2]) shows that

$$
\begin{aligned}
\Phi_{\lambda \lambda^{\prime}}^{q}\left(X_{j}^{N}\right) & =\Phi_{\lambda \lambda^{\prime}}^{q}\left(X_{j}\right)^{N}=\left(X_{j}+q X_{i} X_{j}\right)^{N} \\
& =X_{j}^{N}+\left(q X_{i} X_{j}\right)^{N}=X_{j}^{N}+q^{N} q^{N(N-1)} X_{i}^{N} X_{j}^{N} \\
& =X_{j}^{N}+X_{i}^{N} X_{j}^{N} .
\end{aligned}
$$

Indeed, most of the quantum binomial coefficients are 0 since $q^{2}$ is a primitive $N$-th root of unity. Note that we also used our hypothesis that $q^{N}=(-1)^{N+1}$ for the last equality. It follows that $x_{j}^{\prime}=x_{j}+x_{i} x_{j}=\left(1+x_{i}\right) x_{j}$.

To compute $x_{k}$, it is easier to consider

$$
\begin{aligned}
\Phi_{\lambda \lambda^{\prime}}^{q}\left(X_{k}^{-N}\right) & =\Phi_{\lambda \lambda^{\prime}}^{q}\left(X_{k}\right)^{-N}=\left(X_{k}^{-1}+q X_{k}^{-1} X_{i}^{-1}\right)^{N} \\
& =X_{k}^{-N}+\left(q X_{k}^{-1} X_{i}^{-1}\right)^{N}=X_{k}^{-N}+q^{N} q^{N(N-1)} X_{k}^{-N} X_{i}^{-N} \\
& =X_{k}^{-N}+X_{k}^{-N} X_{i}^{-N} .
\end{aligned}
$$

Applying Lemma 26, we conclude that $x_{k}^{\prime}=\left(x_{k}^{-1}+x_{k}^{-1} x_{i}^{-1}\right)^{-1}=\left(1+x_{i}^{-1}\right)^{-1} x_{k}$. Similar computations hold for $x_{l}^{\prime}$ and $x_{m}^{\prime}$. We conclude that $x_{i}^{\prime}=x_{i}^{-1}, x_{j}^{\prime}=\left(1+x_{i}\right) x_{j}$, $x_{k}^{\prime}=\left(1+x_{i}^{-1}\right)^{-1} x_{k}, x_{l}^{\prime}=\left(1+x_{i}\right) x_{l}, x_{m}^{\prime}=\left(1+x_{i}^{-1}\right)^{-1} x_{m}$ and $x_{h}^{\prime}=x_{h}$ if $h \notin\{i, j, k, l, m\}$. By definition of $\Phi_{\lambda \lambda^{\prime}}^{1}$, this just means that $\rho_{\lambda^{\prime}}^{1}=\rho_{\lambda}^{1} \circ \Phi_{\lambda \lambda^{\prime}}^{1}$.

This completes the proof for an embedded diagonal exchange.

We now consider non-embedded diagonal exchanges. Keeping the same labelling conventions as before, suppose that we are in the case called Case 2 earlier, namely where $\lambda_{j}=\lambda_{l}$ and $\lambda_{k} \neq \lambda_{m}$. In this situation, $X_{j} X_{i}=q^{4} X_{i} X_{j}$ in $\mathcal{T}_{\lambda}^{q}$, which obliges us to use different arguments according to the parity of $N$.

If $N$ is odd, then $q^{4}$ is still a primitive $N$-th root of unity, and the quantum binomial formula again shows that

$$
\begin{aligned}
\Phi_{\lambda \lambda^{\prime}}^{q}\left(X_{j}^{N}\right) & =\left(\left(1+q X_{i}\right)\left(1+q^{3} X_{i}\right) X_{j}\right)^{N}=\left(U+q X_{i} U\right)^{N} \\
& =U^{N}+\left(q X_{i} U\right)^{N}=U^{N}+q^{N} q^{2 N(N-1)} X_{i}^{N} U^{N} \\
& =\left(1+X_{i}^{N}\right) U^{N}
\end{aligned}
$$

where $U=\left(1+q^{3} X_{i}\right) X_{j}$; note for this that $U X_{i}=q^{4} X_{i} U$, and also use $q^{N}=$ $(-1)^{N+1}=1$. Another application of the quantum binomial formula gives

$$
\begin{aligned}
U^{N} & =\left(X_{j}+q^{3} X_{i} X_{j}\right)^{N}=X_{j}^{N}+\left(q^{3} X_{i} X_{j}\right)^{N} \\
& =X_{j}^{N}+q^{3 N} q^{2 N(N-1)} X_{i}^{N} X_{j}^{N}=\left(1+X_{i}^{N}\right) X_{j}^{N}
\end{aligned}
$$


so that $\Phi_{\lambda \lambda^{\prime}}\left(X_{j}^{N}\right)=\left(1+X_{i}^{N}\right)^{2} X_{j}^{N}$. This implies that $x_{j}^{\prime}=\left(1+x_{i}\right)^{2} x_{j}$. The same computations as in the embedded diagonal exchange case give $x_{i}^{\prime}=x_{i}^{-1}, x_{k}^{\prime}=$ $\left(1+x_{i}^{-1}\right)^{-1} x_{k}, x_{m}^{\prime}=\left(1+x_{i}^{-1}\right)^{-1} x_{m}$ and $x_{h}^{\prime}=x_{h}$ if $h \notin\{i, j, k, l, m\}$. By definition of $\Phi_{\lambda \lambda^{\prime}}^{1}$, this implies that $\rho_{1}^{\prime}=\rho_{1} \circ \Phi_{\lambda \lambda^{\prime}}^{1}$ in this case as well.

When $N$ is even, there is a new twist because $q^{4}$ is now a primitive $\frac{N}{2}$-th root of unity. For $U$ as above, the quantum binomial formula gives in this case

$$
\begin{aligned}
\Phi_{\lambda \lambda^{\prime}}^{q}\left(X_{j}^{\frac{N}{2}}\right) & =\left(\left(1+q X_{i}\right)\left(1+q^{3} X_{i}\right) X_{j}\right)^{\frac{N}{2}}=\left(U+q X_{i} U\right)^{\frac{N}{2}} \\
& =U^{\frac{N}{2}}+\left(q X_{i} U\right)^{\frac{N}{2}}=U^{\frac{N}{2}}+q^{\frac{N}{2}} q^{\frac{N(N-2)}{2}} X_{i}^{\frac{N}{2}} U^{\frac{N}{2}} \\
& =\left(1+(-1)^{\frac{N-2}{2}} q^{\frac{N}{2}} X_{i}^{\frac{N}{2}}\right) U^{\frac{N}{2}}
\end{aligned}
$$

and

$$
\begin{aligned}
U^{\frac{N}{2}} & =\left(X_{j}+q^{3} X_{i} X_{j}\right)^{\frac{N}{2}}=X_{j}^{\frac{N}{2}}+\left(q^{3} X_{i} X_{j}\right)^{\frac{N}{2}} \\
& =X_{j}^{\frac{N}{2}}+q^{\frac{3 N}{2}} q^{\frac{N(N-2)}{2}} X_{i}^{\frac{N}{2}} X_{j}^{\frac{N}{2}}=\left(1+(-1)^{\frac{N}{2}} q^{\frac{N}{2}} X_{i}^{\frac{N}{2}}\right) X_{j}^{\frac{N}{2}},
\end{aligned}
$$

using the fact that $q^{N}=(-1)^{N+1}=-1$. It follows that $\Phi_{\lambda \lambda^{\prime}}^{q}\left(X_{j}^{\frac{N}{2}}\right)=\left(1-q^{N} X_{i}^{N}\right) X_{j}^{\frac{N}{2}}$ $=\left(1+X_{i}^{N}\right) X_{j}^{\frac{N}{2}}$. Noting that $X_{i}^{N}$ and $X_{j}^{\frac{N}{2}}$ commute, we conclude that $\Phi_{\lambda \lambda^{\prime}}^{q}\left(X_{j}^{N}\right)=$ $\Phi_{\lambda \lambda^{\prime}}^{q}\left(X_{j}^{\frac{N}{2}}\right)^{2}=\left(1+X_{i}^{N}\right)^{2} X_{j}^{N}$ in this case as well. Therefore, $x_{j}^{\prime}=\left(1+x_{i}\right)^{2} x_{j}$, $x_{i}^{\prime}=x_{i}^{-1}, x_{k}^{\prime}=\left(1+x_{i}^{-1}\right)^{-1} x_{k}, x_{m}^{\prime}=\left(1+x_{i}^{-1}\right)^{-1} x_{m}$ and $x_{h}^{\prime}=x_{h}$ if $h \notin\{i, j, k, l, m\}$ as before. This again implies that $\rho_{\lambda^{\prime}}^{1}=\rho_{\lambda^{\prime}}^{1} \circ \Phi_{\lambda \lambda^{\prime}}^{1}$ in this case.

The remaining types of non-embedded diagonal exchanges are treated in the same way, using the above computations.

Note that the conditions that $q^{2}$ is a primitive $N$-th root of unity and $q^{N}=(-1)^{N+1}$ are equivalent to the property that $q$ is a primitive $N$-th root of $(-1)^{N+1}$, which is shorter to state. The combination of Lemmas 27 and 25 immediately gives:

Theorem 28 Let $q$ be a primitive $N$-th root of $(-1)^{N+1}$. If $\rho=\left\{\rho_{\lambda}: \mathcal{T}_{\lambda}^{q} \rightarrow\right.$ $\operatorname{End}(V)\}_{\lambda \in \Lambda(S)}$ is a finite-dimensional irreducible representation of the polynomial core $\mathcal{T}_{S}^{q}$ of the quantum Teichmüller space $\hat{\mathcal{T}}_{S}^{q}$, then the non-quantum shadows of the $\rho_{\lambda}$ form a representation $\rho^{1}=\left\{\rho_{\lambda}^{1}: \mathcal{T}_{\lambda}^{1} \rightarrow \operatorname{End}(\mathbb{C})\right\}_{\lambda \in \Lambda(S)}$ of the non-quantum polynomial core $\mathcal{T}_{S}^{1}$.

We will say that the representation $\rho^{1}$ of the polynomial core $\mathcal{T}_{S}^{1}$ is the non-quantum shadow of the representation $\rho$ of the polynomial core $\mathcal{T}_{\lambda}^{q}$. 
We now show that every representation of the non-quantum polynomial core $\mathcal{T}_{S}^{1}$ is the shadow of several representations of the quantum polynomial core $\mathcal{T}_{S}^{q}$.

Lemma 29 Let the ideal triangulation $\lambda^{\prime}$ be obtained from $\lambda$ by a re-indexing or by a diagonal exchange. Consider an irreducible finite-dimensional representation $\rho_{\lambda}: \mathcal{T}_{\lambda}^{q} \rightarrow \operatorname{End}(V)$, with non-quantum shadow $\rho_{\lambda}^{1}: \mathcal{T}_{\lambda}^{1} \rightarrow \operatorname{End}(\mathbb{C})$. If there exists a non-quantum representation $\rho_{\lambda^{\prime}}^{1}: \mathcal{T}_{\lambda^{\prime}}^{1} \rightarrow \operatorname{End}(\mathbb{C})=\mathbb{C}^{*}$ with $\rho_{\lambda^{\prime}}^{1}=\rho_{\lambda}^{1} \circ \Phi_{\lambda \lambda^{\prime}}^{1}$, then there exists a unique representation $\rho_{\lambda^{\prime}}: \mathcal{T}_{\lambda^{\prime}}^{q} \rightarrow \operatorname{End}(V)$ with $\rho_{\lambda^{\prime}}=\rho_{\lambda} \circ \Phi_{\lambda \lambda^{\prime}}^{q}$ and with shadow $\rho_{\lambda^{\prime}}^{1}$.

Proof The property is immediate for a re-indexing.

Suppose that $\lambda^{\prime}$ is obtained from $\lambda$ by an embedded diagonal exchange along the component $\lambda_{i}$. Label the components of $\lambda$ bounding the square $Q$ where the diagonal exchange takes place as $\lambda_{j}, \lambda_{k}, \lambda_{l}$ and $\lambda_{m}$, as in Figure 1. By inspection of the formulas defining $\Phi_{\lambda \lambda^{\prime}}^{q}, \rho_{\lambda^{\prime}}\left(X_{s}^{ \pm 1}\right)=\rho_{\lambda} \circ \Phi_{\lambda \lambda^{\prime}}^{q}\left(X_{s}^{ \pm 1}\right)$ will be defined if $\rho_{\lambda}\left(1+q X_{i}\right)$ and $\rho_{\lambda}\left(1+q X_{i}^{-1}\right)$ are invertible in $\operatorname{End}(V)$. As in the proof of Lemma 27,

$$
\begin{aligned}
\rho_{\lambda}\left(\left(1+q X_{i}\right) X_{j}\right)^{N} & =\left(1+\rho_{\lambda}\left(X_{i}^{N}\right)\right) \rho_{\lambda}\left(X_{j}^{N}\right) \\
& =\left(1+\rho_{\lambda}^{1}\left(X_{i}\right)\right) \rho_{\lambda}^{1}\left(X_{j}\right) \operatorname{Id}_{V} \\
& =\rho_{\lambda^{\prime}}^{1}\left(X_{j}\right) \operatorname{Id}_{V} .
\end{aligned}
$$

Since $\rho_{\lambda^{\prime}}^{1}\left(X_{j}\right) \neq 0$, it follows that $\rho_{\lambda}\left(\left(1+q X_{i}\right) X_{j}\right)$ is invertible, and therefore so is $\rho_{\lambda}\left(\left(1+q X_{i}\right)\right)$. A similar consideration of $\rho_{\lambda}\left(X_{k}^{-1}\left(1+q X_{i}^{-1}\right)\right)^{N}$ proves the invertibility of $\rho_{\lambda}\left(1+q X_{i}^{-1}\right)$.

This defines $\rho_{\lambda^{\prime}}$ on the generators $X_{s}^{ \pm 1}$. By inspection, it is compatible with the skew-commutativity relations $X_{s} X_{t}=q^{2 \sigma_{s t}} X_{t} X_{s}$ and consequently extends to an algebra homomorphism $\rho_{\lambda^{\prime}}: \mathcal{T}_{\lambda^{\prime}}^{q} \rightarrow \operatorname{End}(V)$. Its non-quantum shadow is equal to $\rho_{\lambda^{\prime}}^{1}$.

The case of a non-embedded diagonal exchange is treated in the same way, applying again the computations of the proof of Lemma 27.

Theorem 30 Let $q$ be a primitive $N$-th root of $(-1)^{N+1}$. Up to isomorphism, every representation $\rho^{1}=\left\{\rho_{\lambda}^{1}: \mathcal{T}_{\lambda}^{1} \rightarrow \operatorname{End}(\mathbb{C})\right\}_{\lambda \in \Lambda(S)}$ of the non-quantum polynomial core $\mathcal{T}_{S}^{1}$ is the non-quantum shadow of exactly $N^{p}$ if $N$ is odd, and $2^{2 g} N^{p}$ is $N$ is even, irreducible finite-dimensional representations $\rho=\left\{\rho_{\lambda}: \mathcal{T}_{\lambda}^{q} \rightarrow \operatorname{End}(V)\right\}_{\lambda \in \Lambda(S)}$ of the polynomial core $\mathcal{T}_{\lambda}^{q}$, where $p$ is the number of punctures of $S$ and $g$ is its genus. 
Proof Fix an ideal triangulation $\lambda$. By Theorem 21 or 22, according to the parity of $N$, there are $N^{p}$ or $2^{2 g} N^{p}$ isomorphism classes of irreducible finite-dimensional representations $\rho_{\lambda}: \mathcal{T}_{\lambda}^{q} \rightarrow \operatorname{End}(V)$ with non-quantum shadow $\rho_{\lambda}^{1}$. The combination of Lemmas 25 and 29 shows that each such representation $\rho_{\lambda}$ uniquely extends to a representation of the polynomial core $\mathcal{T}_{S}^{q}$.

\section{Pleated surfaces and the hyperbolic shadow of a represen- tation}

We have just showed that the representation theory of the polynomial core $\mathcal{T}_{S}^{q}$ is, up to finitely many ambiguities, controlled by the representation theory of the non-quantum polynomial core $\mathcal{T}_{S}^{1}$. It is now time to remember that the non-quantum coordinate changes $\Phi_{\lambda \lambda^{\prime}}^{1}$ were specially designed to mimic the coordinate changes between shear coordinates for the Teichmüller space of the surface $S$, or more precisely for the enhanced Teichmüller space as defined in [25]. We are going to take advantage of this geometric context.

However, when considering the weights associated to a non-quantum representation, we subreptitiously moved from real to complex numbers. This leads us to consider the complexification of the Teichmüller space, when considered as a real analytic manifold. This complexification has a nice geometric interpretation, based on the fact that the complexification of the orientation-preserving isometry group $\operatorname{PSL}_{2}(\mathbb{R})$ of the hyperbolic plane $\mathbb{T}^{2}$ is the orientation-preserving isometry group $\operatorname{PSL}_{2}(\mathbb{C})$ of the hyperbolic 3-space $\mathbb{M}^{3}$. For this, we will use the technical tool of pleated surfaces, which is now classical in 3-dimensional hyperbolic geometry $[37 ; 11 ; 8]$.

Let $\lambda$ be an ideal triangulation of the surface $S$. A pleated surface with pleating locus $\lambda$ is a pair $(\tilde{f}, r)$, where $\tilde{f}: \widetilde{S} \rightarrow \mathbb{H}^{3}$ is a map from the universal covering $\widetilde{S}$ of $S$ to the hyperbolic 3-space $\mathbb{H}^{3}$, and where $r: \pi_{1}(S) \rightarrow \mathrm{PSL}_{2}(\mathbb{C})$ is a group homomorphism from the fundamental group of $S$ to the group of orientation-preserving isometries of $\mathbb{M}^{3}$, such that:

(1) $\tilde{f}$ homeomorphically sends each component of the preimage $\tilde{\lambda}$ of $\lambda$ to a complete geodesic of $\mathbb{H}^{3}$;

(2) $\tilde{f}$ homeomorphically sends the closure of each component of $\widetilde{S}-\tilde{\lambda}$ to an ideal triangle in $\mathbb{H}^{3}$, namely one whose three vertices are on the sphere at infinity $\partial_{\infty} \mathbb{H}^{3}$ of $\mathbb{H}^{3}$;

(3) $\tilde{f}$ is $r$-equivariant in the sense that $\tilde{f}(\gamma \tilde{x})=r(\gamma) \tilde{f}(\tilde{x})$ for every $\tilde{x} \in \tilde{S}$ and $\gamma \in \pi_{1}(S)$. 
In classical examples arising from geometry, the homomorphism $r$ has discrete image, so that $\tilde{f}$ induces a map $f: S \rightarrow \mathbb{M}^{3} / r\left(\pi_{1}(S)\right)$ to the quotient orbifold $\mathbb{M}^{3} / r\left(\pi_{1}(S)\right)$. The map $f$ is totally geodesic on $S-\lambda$, and is bent along a geodesic ridge at the components of $\lambda$.

The geometry of the pleated surface $(\tilde{f}, r)$ is completely described by complex numbers $x_{i} \in \mathbb{C}^{*}$ associated to the components $\lambda_{i}$ as follows. Consider the upper half-space model for $\mathbb{M}^{3}$, bounded by the Riemann sphere $\widehat{\mathbb{C}}=\mathbb{C} \cup\{\infty\}$. Arbitrarily orient $\lambda_{i}$ and lift it to an oriented component $\tilde{\lambda}_{i}$ of $\tilde{\lambda}$. Let $T_{1}$ be the component of $\widetilde{S}-\tilde{\lambda}$ that is on the left of $\tilde{\lambda}_{i}$, and let $T_{\mathrm{r}}$ be the component on the right, defined with respect to the orientations of $\widetilde{\lambda}_{i}$ and $\widetilde{S}$. Let $z_{+}$and $z_{-} \in \widehat{\mathbb{C}}$ be the positive and negative end points of the oriented geodesic $\tilde{f}\left(\tilde{\lambda}_{i}\right)$ of $\mathbb{M}^{3}$, let $z_{1}$ be the vertex of the ideal triangle $\tilde{f}\left(T_{1}\right)$ that is different from $z_{ \pm}$and, likewise, let $z_{\mathrm{r}}$ be the third vertex of $T_{\mathrm{r}}$. Then $x_{i}$ is defined as the cross-ratio

$$
x_{i}=-\frac{\left(z_{1}-z_{+}\right)\left(z_{\mathrm{r}}-z_{-}\right)}{\left(z_{1}-z_{-}\right)\left(z_{\mathrm{r}}-z_{+}\right)}
$$

Note that $x_{i}$ is different from 0 and $\infty$, because the vertex sets $\left\{z_{+}, z_{-}, z_{1}\right\}$ and $\left\{z_{+}, z_{-}, z_{\mathrm{r}}\right\}$ of the ideal triangles $\tilde{f}\left(T_{1}\right)$ and $\tilde{f}\left(T_{\mathrm{r}}\right)$ each consist of three distinct points. Also, reversing the orientation of $\lambda_{i}$ exchanges $z_{+}$and $z_{-}$, but also exchanges $z_{1}$ and $z_{\mathrm{r}}$ so that $x_{i}$ is unchanged. Similarly, $x_{i}$ is independent of the choice of the lift $\tilde{\lambda}_{i}$ by invariance of cross-ratios under hyperbolic isometries.

By definition, $x_{i} \in \mathbb{C}^{*}$ is the exponential shear-bend parameter of the pleated surface $(\widetilde{f}, r)$ along the component $\lambda_{i}$ of $\lambda$. Geometrically, the imaginary part of $\log x_{i}$ (defined modulo $2 \pi \mathrm{i}$ ) is the external dihedral angle of the ridge formed by $\widetilde{f}(\widetilde{S})$ near the preimage of $\lambda_{i}$. The real part of $\log x_{i}$ is the oriented distance from $z_{1}^{\prime}$ to $z_{\mathrm{r}}^{\prime}$ in the oriented geodesic $\tilde{f}\left(\tilde{\lambda}_{i}\right)$, where $z_{1}^{\prime}$ and $z_{\mathrm{r}}^{\prime}$ are the respective orthogonal projections of $z_{1}$ and $z_{\mathrm{r}}$ to $\tilde{f}\left(\tilde{\lambda}_{i}\right)$. See for instance [8].

Two pleated surfaces $(\tilde{f}, r)$ and $\left(\tilde{f}^{\prime}, r^{\prime}\right)$ are isometric if there is a hyperbolic isometry $A \in \mathrm{PSL}_{2}(\mathbb{C})$ and a lift $\tilde{\varphi}: \widetilde{S} \rightarrow \widetilde{S}$ of an isotopy of $S$ such that $\tilde{f}^{\prime}=A \circ \tilde{f} \circ \tilde{\varphi}$ and $r^{\prime}(\gamma)=\operatorname{Ar}(\gamma) A^{-1}$ for every $\gamma \in \pi_{1}(S)$.

Proposition 31 For a given ideal triangulation, two pleated surfaces $(\widetilde{f}, r)$ and $\left(\tilde{f}^{\prime}, r^{\prime}\right)$ with pleating locus $\lambda$ are isometric if and only if they have the same exponential shear-bend factors $x_{i} \in \mathbb{C}^{*}$ at the components $\lambda_{i}$ of $\lambda$. Conversely, any set of weights $x_{i} \in \mathbb{C}^{*}$ on the components $\lambda_{i}$ of $\lambda$ can be realized as the exponential shear-bend parameters of a pleated surface $(\tilde{f}, r)$ with pleating locus $\lambda$. 
Note that, for a pleated surface $(\tilde{f}, r)$, the homomorphism $r: \pi_{1}(S) \rightarrow \operatorname{PSL}_{2}(\mathbb{C})$ is completely determined by the map $\tilde{f}: \tilde{S} \rightarrow \mathbb{M}^{3}$. The map $\tilde{f}$ adds more data to $r$ as follows. Let $A \subset S$ be the union of small annulus neighborhoods of all the punctures of $S$. There is a one-to-one correspondence between the components of the preimage $\tilde{A}$ of $A$ and the peripheral subgroups of $\pi_{1}(S)$, namely of the images of the homomorphisms $\pi_{1}(A) \rightarrow \pi_{1}(S)$ defined by all possible choices of base points and paths joining these base points. For a component $\tilde{A}_{\pi}$ of $\tilde{A}$ corresponding to a peripheral subgroup $\pi \subset \pi_{1}(S)$, the images under $\tilde{f}$ of the triangles of $\tilde{S}-\tilde{\lambda}$ that meet $\tilde{A}_{\pi}$ all have a vertex $z_{\pi}$ in common in $\widehat{\mathbb{C}}=\partial_{\infty} \mathbb{H}^{3}$, and this vertex is fixed by $r(\pi)$. Therefore, $\tilde{f}$ associates to each peripheral subgroup $\pi$ of $\pi_{1}(S)$ a point $z_{\pi} \in \partial_{\infty} \mathbb{H}^{3}$ which is fixed under $r(\pi)$. In addition this assignment is $r$-equivariant in the sense that $z_{\gamma \pi \gamma^{-1}}=r(\gamma) z_{\pi}$ for every $\gamma \in \pi_{1}(S)$.

By definition, an enhanced homomorphism $\left(r,\left\{z_{\pi}\right\}_{\pi \in \Pi}\right)$ of $\pi_{1}(S)$ in $\mathrm{PSL}_{2}(\mathbb{C})$ consists of a group homomorphism $r: \pi_{1}(S) \rightarrow \mathrm{PSL}_{2}(\mathbb{C})$ together with an $r$-equivariant assignment of a fixed point $z_{\pi} \in \partial_{\infty} \mathbb{H}^{3}$ to each peripheral subgroup $\pi$ of $\pi_{1}(S)$. Here $\Pi$ denotes the set of peripheral subgroups of $\pi_{1}(S)$. By abuse of notation, we will often write $r$ instead of $\left(r,\left\{z_{\pi}\right\}_{\pi \in \Pi}\right)$ of $\pi_{1}(S)$.

In general, a homomorphism $r: \pi_{1}(S) \rightarrow \mathrm{PSL}_{2}(\mathbb{C})$ admits few possible enhancements. Indeed, if the peripheral subgroup $r(\pi)$ is parabolic, it fixes only one point in $\partial_{\infty} \mathbb{H}^{3}$ and $z_{\pi}$ is therefore uniquely determined by $r$. If $r(\pi)$ is loxodromic or elliptic, there are exactly two possible choices for $z_{\pi}$, namely the end points of the axis of $r(\pi)$; choosing one of these points as $z_{\pi}$ therefore amounts to choosing an orientation for the axis of $r(\pi)$. The only case where there are many possible choices for $z_{\pi}$ is when $r(\pi)$ is the identity, which is highly non-generic.

When all the exponential shear-bend parameters $x_{i} \in \mathbb{C}^{*}$ are positive real, there is no bending and the associated pleated surface $\tilde{f}$ immerses $\widetilde{S}$ in a hyperbolic plane in $\mathbb{H}^{3}$. In particular, the associated pleated surface $(\tilde{f}, r)$ can be chosen so that the image of $r$ is contained in the isometry group $\mathrm{PSL}_{2}(\mathbb{R})$ of the hyperbolic plane $\mathbb{H}^{2}$. It can be shown that $r: \pi_{1}(S) \rightarrow \mathrm{PSL}_{2}(\mathbb{R})$ is injective and has discrete image, and that each peripheral subgroup is either parabolic or loxodromic; see for instance [41, Section 3.4]. In particular, the enhanced homomorphism $r$ defines an element of the enhanced Teichmüller space of $S$, in the terminology of [25]. The positive real parameters $x_{i}$ are by definition the exponential shear coordinates for the enhanced Teichmüller space of $S$.

Given an ideal triangulation $\lambda$, Proposition 31 and the above observations associate to a non-quantum representation $\rho_{\lambda}^{1}: \mathcal{T}_{\lambda}^{1} \rightarrow \operatorname{End}(\mathbb{C})$ an enhanced homomorphism 
$r_{\lambda}: \pi_{1}(S) \rightarrow \mathrm{PSL}_{2}(\mathbb{C})$. This correspondence is particularly well-behaved as we move from one ideal triangulation to another.

Lemma 32 Let the ideal triangulation $\lambda^{\prime}$ be obtained from $\lambda$ by a re-indexing or a diagonal exchange, and consider two non-quantum representations $\rho_{\lambda}^{1}: \mathcal{T}_{\lambda}^{1} \rightarrow \operatorname{End}(\mathbb{C})$ and $\rho_{\lambda^{\prime}}^{1}: \mathcal{T}_{\lambda^{\prime}}^{1} \rightarrow \operatorname{End}(\mathbb{C})$ such that $\rho_{\lambda^{\prime}}^{1}=\rho_{\lambda}^{1} \circ \Phi_{\lambda \lambda^{\prime}}^{1}$. Then the pleated surfaces $\left(\tilde{f_{\lambda}}, r_{\lambda}\right)$ and $\left(\widetilde{f}_{\lambda^{\prime}}, r_{\lambda^{\prime}}\right)$ respectively associated to $\rho_{\lambda}^{1}$ and $\rho_{\lambda^{\prime}}^{1}$ define the same enhanced homomorphism $r_{\lambda}=r_{\lambda^{\prime}}: \pi_{1}(S) \rightarrow \mathrm{PSL}_{2}(\mathbb{C})$, up to conjugation by an element of $\mathrm{PSL}_{2}(\mathbb{C})$.

Proof The property is immediate when $\lambda^{\prime}$ is obtained by re-indexing the components of $\lambda$. We can therefore suppose that $\lambda^{\prime}$ is obtained from $\lambda$ by a diagonal exchange along the component $\lambda_{i}$.

For a component $\tilde{\lambda}_{i}$ of the preimage of $\lambda_{i}$, consider as before the left and right components $T_{1}$ and $T_{\mathrm{r}}$ of $\widetilde{S}-\tilde{\lambda}$ that are adjacent to $\lambda_{i}$, the end points $z_{+}$and $z_{-}$ of $\tilde{f}_{\lambda}\left(\tilde{\lambda}_{i}\right)$, and the remaining vertices $z_{1}$ and $z_{\mathrm{r}}$ of the triangles $\tilde{f}_{\lambda}\left(T_{1}\right)$ and $\tilde{f}_{\lambda}\left(T_{\mathrm{r}}\right)$. Let $Q\left(\widetilde{\lambda}_{i}\right) \subset \widetilde{S}$ be the open square $T_{1} \cup T_{\mathrm{r}} \cup \tilde{\lambda}_{i}$; it admits $\tilde{\lambda}_{i}$ as a diagonal, but also a component $\tilde{\lambda}_{i}^{\prime}$ of $\tilde{\lambda}^{\prime}$ as another diagonal.

Because $\rho_{\lambda^{\prime}}^{1}=\rho_{\lambda}^{1} \circ \Phi_{\lambda \lambda^{\prime}}^{1}$ is well-defined, the exponential shear-bend parameter $x_{i} \in \mathbb{C}^{*}$ of $\left(\tilde{f}_{\lambda}, r_{\lambda}\right)$ along $\lambda_{i}$ is different from -1 . This implies that the points $z_{1}$ and $z_{\mathrm{r}}$ are distinct. We can therefore modify $\tilde{f}_{\lambda}$ on $Q\left(\tilde{\lambda}_{i}\right)$ so that it sends the diagonal $\tilde{\lambda}_{i}^{\prime}$ to the geodesic of $\mathbb{H}^{3}$ joining $z_{1}$ to $z_{\mathrm{r}}$, and the square $Q\left(\tilde{\lambda}_{i}\right)$ to the union of the ideal triangles with respective vertex sets $\left\{z_{1}, z_{\mathrm{r}}, z_{+}\right\}$and $\left\{z_{1}, z_{\mathrm{r}}, z_{-}\right\}$. As $\tilde{\lambda}_{i}$ ranges over all the components of the preimage of $\lambda_{i}$ in $\tilde{\lambda}$, the corresponding squares $Q\left(\tilde{\lambda}_{i}\right)$ are pairwise disjoint, and we can therefore perform this operation equivariantly with respect to $r_{\lambda}$. This gives a pleated surface $\left(\tilde{f}_{\lambda}^{\prime}, r_{\lambda}\right)$ with pleating locus $\lambda^{\prime}$ and with the same holonomy $r_{\lambda}: \pi_{1}(S) \rightarrow \mathrm{PSL}_{2}(\mathbb{C})$ as the original pleated surface $\left(\tilde{f}_{\lambda}, r_{\lambda}\right)$. Note that $\left(\widetilde{f}_{\lambda}^{\prime}, r_{\lambda}\right)$ even has the same associated enhanced homomorphism as $\left(\tilde{f}_{\lambda}, r_{\lambda}\right)$.

It remains to show that the exponential shear-bend parameters of $\left(\tilde{f}_{\lambda}^{\prime}, r_{\lambda}\right)$ are the numbers $x_{i}^{\prime} \in \mathbb{C}^{*}$ associated to the non-quantum representation $\rho_{\lambda^{\prime}}^{1}=\rho_{\lambda}^{1} \circ \Phi_{\lambda_{\lambda^{\prime}}}^{1}: \mathcal{T}_{\lambda^{\prime}}^{1} \rightarrow$ End $(\mathbb{C})$. The coordinate change isomorphism $\Phi_{\lambda \lambda^{\prime}}^{1}: \mathcal{T}_{\lambda^{\prime}}^{1} \rightarrow \mathcal{T}_{\lambda}^{1}$ was specially designed so that, when the $x_{i}$ are real positive and correspond to shear coordinates of the enhanced Teichmüller space, it exactly reflects the corresponding change of shear coordinates for the enhanced Teichmüller space; see for instance [25]. The corresponding combinatorics of cross-ratios automatically extend to the complex case, and guarantees that the nonquantum representation $\mathcal{T}_{\lambda^{\prime}}^{1} \rightarrow \operatorname{End}(\mathbb{C})$ defined by the $x_{i}^{\prime}$ is exactly $\rho_{\lambda^{\prime}}^{1}=\rho_{\lambda}^{1} \circ \Phi_{\lambda \lambda^{\prime}}^{1}$.

As a consequence, $\left(\tilde{f}_{\lambda}^{\prime}, r_{\lambda}\right)$ is isometric to $\left(\tilde{f}_{\lambda^{\prime}}, r_{\lambda^{\prime}}\right)$, which concludes the proof. 
Proposition 33 Every representation $\rho^{1}=\left\{\rho_{\lambda}^{1}: \mathcal{T}_{\lambda}^{1} \rightarrow \operatorname{End}(\mathbb{C})\right\}_{\lambda \in \Lambda(S)}$ of the nonquantum polynomial core $\mathcal{T}_{S}^{1}$ uniquely determines an enhanced homomorphism $r$ : $\pi_{1}(S) \rightarrow \mathrm{PSL}_{2}(\mathbb{C})$ such that, for every ideal triangulation $\lambda \in \Lambda(S), r$ is the enhanced homomorphism associated to the pleated surface with bending locus $\lambda$ and with exponential shear bend parameters $\rho_{\lambda}^{1}\left(X_{i}\right) \in \mathbb{C}^{*}$, for $i=1, \ldots, n$. Conversely, two representations of $\mathcal{T}_{S}^{1}$ that induce the same enhanced homomorphism $r: \pi_{1}(S) \rightarrow \mathrm{PSL}_{2}(\mathbb{C})$ must be equal.

Proof The first statement is an immediate consequence of Lemma 32.

To prove the second statement, suppose that the two representations $\rho$ and $\rho^{\prime}$ of $\mathcal{T}_{S}^{1}$ induce the same enhanced homomorphism, consisting of a homomorphism $r: \pi_{1}(S) \rightarrow$ $\mathrm{PSL}_{2}(\mathbb{C})$ and of an $r$-equivariant family of fixed points $z_{\pi}$ associated to the peripheral subgroups $\pi$ of $\pi_{1}(S)$. Let $\left(\tilde{f}_{\lambda}, r_{\lambda}\right)$ and $\left(\tilde{f}_{\lambda}^{\prime}, r_{\lambda}^{\prime}\right)$ be the two pleated surfaces with bending locus $\lambda$ respectively associated to $\rho$ and $\rho^{\prime}$. After isometries, we can arrange that $r_{\lambda}=r_{\lambda}^{\prime}=r$.

Each end of a component $\tilde{\lambda}_{i}$ of the preimage $\tilde{\lambda} \subset \widetilde{S}$ specifies two peripheral subgroups $\pi$ and $\pi^{\prime}$ of $\pi_{1}(S)$. By construction $\tilde{f}_{\lambda}$ and $\tilde{f}_{\lambda}^{\prime}$ must both send $\widetilde{\lambda}_{i}$ to the geodesic of $\mathbb{H}^{3}$ joining the two points $z_{\pi}$ and $z_{\pi^{\prime}}$. After a $\pi_{1}(S)$-equivariant isotopy of $\widetilde{S}$, one can arrange that $\tilde{f}_{\lambda}$ and $\widetilde{f}_{\lambda}^{\prime}$ coincide on $\tilde{\lambda}$, and eventually over all of $\widetilde{S}$ by adjustment on the triangle components of $\tilde{S}-\tilde{\lambda}$. In particular, the two pleated surfaces $\tilde{f}_{\lambda}$ and $\widetilde{f}_{\lambda}^{\prime}$ now coincide. Since these pleated surfaces now have the same exponential shear-bend parameters, it follows that $\rho$ and $\rho^{\prime}$ coincide on $\mathcal{T}_{\lambda}^{1}$, and therefore over all of $\mathcal{T}_{S}^{1}$.

By definition, the enhanced homomorphism $r: \pi_{1}(S) \rightarrow \mathrm{PSL}_{2}(\mathbb{C})$ provided by Proposition 33 is the hyperbolic shadow of the non-quantum representation $\rho^{1}$. In the case where $\rho^{1}$ is the non-quantum shadow of a representation $\rho$ of the polynomial core $\mathcal{T}_{S}^{q}$ of the quantum Teichmüller space (for a primitive $N$-th root $q$ of $(-1)^{N+1}$ ), we will also say that $r$ is the hyperbolic shadow of $\rho$.

Not every enhanced homomorphism from $\pi_{1}(S)$ to $\mathrm{PSL}_{2}(\mathbb{C})$ is associated to a representation of the polynomial core $\mathcal{T}_{S}^{1}$ as above. However, many geometrically interesting ones are.

Lemma 34 Consider an injective homomorphism $r: \pi_{1}(S) \rightarrow \mathrm{PSL}_{2}(\mathbb{C})$. Then, every enhancement of $r$ is the hyperbolic shadow of a representation $\rho^{1}$ of the non-quantum polynomial core $\mathcal{T}_{S}^{1}$.

Proof The key property is that the stabilizer of a point $z \in \partial_{\infty} \mathbb{H}^{3}$ in $\mathrm{PSL}_{2}(\mathbb{C})$ is solvable, whereas two distinct peripheral subgroups of $\pi_{1}(S)$ generate a free subgroup of 
rank 2, which cannot be contained in a solvable group. It follows that any enhancement of $r$ associates distinct points $z_{\pi}$ and $z_{\pi^{\prime}}$ to distinct peripheral subgroups $\pi$ and $\pi^{\prime}$.

Let $\lambda$ be an arbitrary ideal triangulation of $S$, with preimage $\tilde{\lambda}$ in the universal covering $\widetilde{S}$. The corners of each component $T$ of $\widetilde{S}-\widetilde{\lambda}$ specify three distinct peripheral subgroups $\pi_{1}^{T}, \pi_{2}^{T}$ and $\pi_{3}^{T}$. We can then construct a pleated surface $\left(\tilde{f}_{\lambda}, r\right)$ with pleating locus $\lambda$, equivariant with respect to the given representation $r$, which sends each component $T$ of $\tilde{S}-\tilde{\lambda}$ to the ideal triangle of $\mathbb{H}^{3}$ with vertices $z_{\pi_{1}^{T}}, z_{\pi_{2}^{T}}$, $z_{\pi_{3}^{T}} \in \partial_{\infty} \mathbb{H}^{3}$. The pleated surface $\left(\tilde{f_{\lambda}}, r\right)$ defines a representation $\rho_{\lambda}^{1}: \mathcal{T}_{\lambda}^{1} \rightarrow \operatorname{End}(\mathbb{C})$ whose associated enhanced homomorphism consists of $r$ and the $z_{\pi}$.

As $\lambda$ ranges over all ideal triangulations, (the proof of) Lemma 32 shows that the $\rho_{\lambda}^{1}$ fit together to provide a representation $\rho^{1}$ of the polynomial core $\mathcal{T}_{S}^{1}$ whose associated enhanced representation consists of $r$ and the $z_{\pi}$.

An injective homomorphism $r: \pi_{1}(S) \rightarrow \mathrm{PSL}_{2}(\mathbb{C})$ admits $2^{l}$ enhancements, where $l$ is the number of ends of $S$ whose image under $r$ is loxodromic. Combining Theorem 30, Proposition 33 and Lemma 34 immediately gives:

Theorem 35 Let $q$ be a primitive $N$-th root of $(-1)^{N+1}$. Up to isomorphism, an injective homomorphism $r: \pi_{1}(S) \rightarrow \mathrm{PSL}_{2}(\mathbb{C})$ is the hyperbolic shadow of $2^{l} N^{p}$ if $N$ is odd, and $2^{2 g+l} N^{p}$ if $N$ is even, irreducible finite-dimensional representations of the polynomial core $\mathcal{T}_{S}^{q}$ (where $g$ is the genus of $S, p$ is its number of punctures, and $l$ is the number of ends of $S$ whose image under $r$ is loxodromic).

\section{Invariants of surface diffeomorphisms}

Theorem 35 provides a finite-to-one correspondence between representations of the polynomial core $\mathcal{T}_{S}^{q}$ and certain homomorphisms from $\pi_{1}(S)$ to $\operatorname{PSL}_{2}(\mathbb{C})$. We will take advantage of this correspondence to construct interesting representations of the polynomial core by using hyperbolic geometry.

Let $\varphi: S \rightarrow S$ be an orientation-preserving diffeomorphism of the surface $S$. If $\lambda$ is an ideal triangulation of $S, \varphi$ induces a natural isomorphism $\varphi_{\lambda}^{q}: \mathcal{T}_{\lambda}^{q} \rightarrow \mathcal{T}_{\varphi(\lambda)}^{q}$ which, to the $i$-th generator $X_{i}$ of the Chekhov-Fock algebra $\mathcal{T}_{\lambda}^{q}$ corresponding to the component $\lambda_{i}$ of $\lambda$, associates the $i$-th generator $X_{i}^{\prime}$ of $\mathcal{T}_{\varphi(\lambda)}^{q}$ corresponding to the component $\varphi\left(\lambda_{i}\right)$ of $\varphi(\lambda)$. The existence of $\varphi$ guarantees that the $X_{i}$ and $X_{i}^{\prime}$ satisfy the same relations, so that $\varphi_{\lambda}^{q}$ is a well-defined algebra isomorphism.

The isomorphism $\varphi_{\lambda}^{q}$ induces an isomorphism $\hat{\varphi}_{\lambda}^{q}: \hat{\mathcal{T}}_{\lambda}^{q} \rightarrow \hat{\mathcal{T}}_{\varphi(\lambda)}^{q}$ between the corresponding fraction algebras. As $\lambda$ ranges over all ideal triangulations, the $\hat{\varphi}_{\lambda}^{q}$ commute with 
the coordinate change isomorphisms $\Phi_{\lambda \lambda^{\prime}}^{q}$, in the sense that $\hat{\varphi}_{\lambda}^{q} \circ \Phi_{\lambda \lambda^{\prime}}^{q}=\Phi_{\varphi(\lambda) \varphi\left(\lambda^{\prime}\right)}^{q} \circ \hat{\varphi}_{\lambda^{\prime}}^{q}$. The $\hat{\varphi}_{\lambda}^{q}$ consequently define an isomorphism $\hat{\varphi}_{S}^{q}$ of the quantum Teichmüller space $\hat{\mathcal{T}}_{S}^{q}$. Note that $\hat{\varphi}_{S}^{q}$ sends the image of $\mathcal{T}_{\lambda}^{q}$ in $\hat{\mathcal{T}}_{S}^{q}$ to $\mathcal{T}_{\varphi(\lambda)}^{q}$, and therefore induces an isomorphism $\varphi_{S}^{q}$ of the polynomial core $\mathcal{T}_{S}^{q}$.

In particular, $\varphi$ now acts on the set $\mathcal{R}^{q}$ of irreducible finite-dimensional representations of the polynomial cores $\mathcal{T}_{S}^{q}$ by associating to the representation $\rho=\left\{\rho_{\lambda}: \mathcal{T}_{\lambda}^{q} \rightarrow\right.$ $\operatorname{End}(V)\}_{\lambda \in \Lambda(S)}$ the representation $\rho \circ \varphi_{S}^{q}=\left\{\rho_{\varphi(\lambda)} \circ \varphi_{\lambda}^{q}: \mathcal{T}_{\lambda}^{q} \rightarrow \operatorname{End}(V)\right\}_{\lambda \in \Lambda(S)}$.

Lemma 36 If $\rho$ is an irreducible finite-dimensional representation of the polynomial core $\mathcal{T}_{S}^{q}$ and if the enhanced homomorphism $\left(r,\left\{z_{\pi}\right\}_{\pi \in \Pi}\right)$ is its hyperbolic shadow, then the hyperbolic shadow of the representation $\rho \circ \varphi_{S}^{q}$ is equal to $(r \circ$ $\left.\varphi^{*},\left\{z_{\varphi^{*}(\pi)}\right\}_{\pi \in \Pi}\right)$, where $\varphi^{*}: \pi_{1}(S) \rightarrow \pi_{1}(S)$ is the isomorphism induced by the diffeomorphism $\varphi: S \rightarrow S$ for an arbitrary choice of a path joining the base point of $S$ to its image under $\varphi$.

Note that, up to isometry of $\mathbb{M}^{3}$, the enhanced representation $\left(r \circ \varphi^{*},\left\{z_{\varphi^{*}(\pi)}\right\}_{\pi \in \Pi)}\right)$ is independent of the choice of path involved in the definition of $\varphi^{*}$.

Proof of Lemma 36 Let $\tilde{\varphi}: \widetilde{S} \rightarrow \widetilde{S}$ be an arbitrary lift of $\varphi$ to the universal cover $\widetilde{S}$. If $\rho=\left\{\rho_{\lambda}: \mathcal{T}_{\lambda}^{q} \rightarrow \operatorname{End}(V)\right\}_{\lambda \in \Lambda(S)}$ and if $\left(\tilde{f}_{\lambda}, r_{\lambda}\right)$ is the pleated surface with pleating locus $\lambda$ associated to $\rho_{\lambda}$, the pleated surface with pleating locus $\lambda$ associated to $\rho_{\varphi(\lambda)} \circ \varphi_{\lambda}^{q}$ is isometric to $\left(\tilde{f}_{\varphi(\lambda)} \circ \tilde{\varphi}, r_{\varphi(\lambda)} \circ \varphi^{*}\right)$. The result then immediately follows from definitions.

We are now ready to use geometric data to construct special representations of the polynomial core. This construction will require the diffeomorphism $\varphi$ to be homotopically aperiodic (or homotopically pseudo-Anosov) namely such that, for every $n>0$ and every non-trivial $\gamma \in \pi_{1}(S), \varphi_{*}^{n}(\gamma)$ is not conjugate to $\gamma$ in $\pi_{1}(S)$. The Nielsen-Thurston classification of surface diffeomorphisms [40; 14] asserts that every isotopy class of surface diffeomorphism can be uniquely decomposed into pieces that are either periodic or homotopically aperiodic.

There is another characterization of homotopically aperiodic surface diffeomorphisms in terms of the geometry of their mapping torus. The mapping torus $M_{\varphi}$ of the diffeomorphism $\varphi: S \rightarrow S$ is the 3-dimensional manifold quotient of $S \times \mathbb{R}$ by the free action of $\mathbb{Z}$ defined by $n \cdot(x, t)=\left(\varphi^{n}(x), t+n\right)$ for $n \in \mathbb{Z}$ and $(x, t) \in$ $S \times \mathbb{R}$. Thurston's Hyperbolization Theorem [38] asserts that $\varphi$ is homotopically aperiodic if and only if the mapping torus $M_{\varphi}$ admits a complete hyperbolic metric; see [30] for a proof of this statement. When this hyperbolic metric exists, it is unique 
by Mostow's Rigidity Theorem [27], and its holonomy associates to $\varphi$ an injective homomorphism $r_{\varphi}: \pi_{1}\left(M_{\varphi}\right) \rightarrow \mathrm{PSL}_{2}(\mathbb{C})$, uniquely defined up to conjugation by an element of $\mathrm{PSL}_{2}(\mathbb{C})$, for which every peripheral subgroup is parabolic. Consider the map $f: S \rightarrow M_{\varphi}$ composition of the natural identification $S=S \times\{0\} \subset S \times \mathbb{R}$ and of the projection $S \times \mathbb{R} \rightarrow M_{\varphi}=S \times \mathbb{R} / \mathbb{Z}$. For a suitable choice of base points, this enables us to specify a restriction $r_{\varphi}: \pi_{1}(S) \rightarrow \mathrm{PSL}_{2}(\mathbb{C})$ of the holonomy homomorphism of $M_{\varphi}$.

The key property is now that $f$ is homotopic to $f \circ \varphi$ in $M_{\varphi}$. This has the following immediate consequence.

Lemma 37 The homomorphisms $r_{\varphi}$ and $r_{\varphi} \circ \varphi^{*}: \pi_{1}(S) \rightarrow \mathrm{PSL}_{2}(\mathbb{C})$ are conjugate by an element of $\mathrm{PSL}_{2}(\mathbb{C})$.

Since every peripheral subgroup of $\pi_{1}(S)$ is parabolic for $r_{\varphi}$, the homomorphism $r_{\varphi}$ admits a unique enhancement. Let $\mathcal{R}_{\varphi}^{q} \subset \mathcal{R}^{q}$ be the set of (isomorphism classes) of irreducible finite-dimensional representations of the polynomial core $\mathcal{T}_{S}^{q}$ whose hyperbolic shadow is equal to $r_{\varphi}$. By Theorem 35, the set $\mathcal{R}_{\varphi}^{q}$ is finite, and has $N^{p}$ or $2^{2 g} N^{p}$ elements according to whether $N$ is odd or even. By Lemmas 36 and 37, the set $\mathcal{R}_{\varphi}^{q}$ is invariant under the action of $\varphi$.

By finiteness of $\mathcal{R}_{\varphi}^{q}$, for every $\rho=\left\{\rho_{\lambda}: \mathcal{T}_{\lambda}^{q} \rightarrow \operatorname{End}(V)\right\}_{\lambda \in \Lambda(S)}$ in $\mathcal{R}_{\varphi}^{q}$, there is a smallest integer $k \geqslant 1$ such that $\rho \circ\left(\varphi_{S}^{q}\right)^{k}=\rho$ in $\mathcal{R}_{\varphi}^{q}$. This does not mean that the representations $\rho \circ\left(\varphi_{S}^{q}\right)^{k}$ and $\rho$ of the polynomial core $\mathcal{T}_{S}^{q}$ over $V$ coincide, but that there exists an automorphism $L_{\rho}$ of $V$ such that

$$
\rho \circ\left(\varphi_{S}^{q}\right)^{k}(X)=L_{\rho} \cdot \rho(X) \cdot L_{\rho}^{-1}
$$

in $\operatorname{End}(V)$ for every $X \in \mathcal{T}_{S}^{q}$, if we denote by $\cdot$ the composition in $\operatorname{End}(V)$ and by $\circ$ any other composition of maps to avoid confusion.

Proposition 38 The automorphism $L_{\rho}$ of $V$ depends uniquely on the orbit of $\rho \in \mathcal{R}_{\varphi}^{q}$ under $\varphi_{S}^{q}$, up to conjugation by an automorphism of $V$ and scalar multiplication by a non-zero complex number.

Proof By irreducibility of $\rho$, the isomorphism $L_{\rho}$ of $V$ is completely determined up to scalar multiplication by the property that $\rho \circ\left(\varphi_{S}^{q}\right)^{k}(X)=L_{\rho} \cdot \rho_{\lambda}(X) \cdot L_{\rho}^{-1}$ for every $X \in \mathcal{T}_{S}^{q}$. It is also immediate that we can take $L_{\rho \circ \varphi_{S}^{q}}=L_{\rho}$. Finally, one needs to remember that the representation $\rho$ was considered up to isomorphism of representations. A representation isomorphism replaces $L_{\rho}$ by a conjugate. 
We consequently have associated to each orbit of the action of $\varphi$ on $\mathcal{R}_{\varphi}^{q}$ a square matrix $L_{\rho}$ of rank $N^{3 g+p-3}$ or $N^{3 g+p-3} / 2^{g}$, according to wether $N$ is odd or even, which is well-defined up to conjugation and scalar multiplication. It is not too hard to determine these orbits in terms of the action of $\varphi$ on the punctures of $S$ and, when $N$ is even, on $H_{1}\left(S ; \mathbb{Z}_{2}\right)$. However, this process can be cumbersome.

Fortunately, when $N$ is odd, there is preferred fixed point for the action of $\varphi$ on $\mathcal{R}_{\varphi}^{q}$. This is based on the following geometric observation. Recall from Lemma 24 that the central elements $P_{j}$ associated to the punctures of $S$ and the square root $H$ of $P_{1} P_{2} \ldots P_{p}$ are well-defined elements of the polynomial core $\mathcal{T}_{S}^{q}$.

Lemma 39 Let $\rho_{\varphi}^{1}$ be the non-quantum representation of $\mathcal{T}_{S}^{1}$ whose hyperbolic shadow is equal to $r_{\varphi}$. Then $\rho_{\varphi}^{1}$ sends the central elements $H$ and $P_{j}$ to the identity.

Proof Fix an ideal triangulation $\lambda$, and let $\left(\tilde{f}, r_{\varphi}\right)$ be the pleated surface with pleating locus $\lambda$ associated to $r_{\varphi}$.

Consider the $j$-th puncture $v_{j}$ of $S$. Because the corresponding peripheral subgroup of $\pi_{1}(S)$ is parabolic for $r_{\varphi}$, the product of the exponential shear-bend coordinates $x_{i} \in \mathbb{C}^{*}$ associated to the components $\lambda_{i}$ converging towards $v_{j}$ (counted with multiplicity) is equal to 1 ; see for instance [8]. By definition of $P_{j}$, this means that the representation $\mathcal{T}_{\lambda}^{1} \rightarrow \operatorname{End}(\mathbb{C})$ induced by $\rho_{\varphi}^{1}$ sends $P_{j}$ to the identity.

Since $H^{2}=P_{1} P_{2} \ldots P_{p}$, it follows that $\rho_{\varphi}^{1}$ sends $H$ to $\pm 1= \pm \operatorname{Id}_{\mathbb{C}}$. By construction [30], the homomorphism $r_{\varphi}$ is in the same component as the fuchsian homomorphisms in the space of injective homomorphisms $r: \pi_{1}(S) \rightarrow \mathrm{PSL}_{2}(\mathbb{C})$. For a fuchsian homomorphism, all the $x_{i}$ are real positive, so that $\rho_{r}^{1}(H)=+1=\operatorname{Id}_{\mathbb{C}}$ for the associated representation. By connectedness, it follows that $\rho_{\varphi}^{1}(H)=+1=\operatorname{Id}_{\mathbb{C}}$.

When $N$ is odd, we can paraphrase Theorem 21 by saying that a representation $\rho$ of the Chekhov-Fock algebra $\mathcal{T}_{\lambda}^{q}$ is classified by its non-quantum shadow $\rho^{1}: \mathcal{T}_{\lambda}^{1} \rightarrow$ $\operatorname{End}(\mathbb{C})=\mathbb{C}^{*}$ and by the choice of an $N$-th root for $\rho^{1}(H)$ and for each of the $\rho^{1}\left(P_{j}\right)$. In the case when $\rho^{1}=\rho_{\varphi}^{1}$, Lemma 39 provides an obvious choice for these $N$-th roots, namely 1 . Therefore, $r_{\varphi}$ specifies a unique representation $\rho_{\varphi}$ of the polynomial core $\mathcal{T}_{S}^{q}$ over a vector space $V$ of dimension $N^{3 g+p-3}$, for which $\rho_{\varphi}(H)=\rho_{\varphi}\left(P_{j}\right)=\operatorname{Id}_{V}$. We can paraphrase this last condition by saying that $\rho_{\varphi}$ induces a representation of the quantum cusped Teichmüller space, as defined in [25].

Since the action of $\varphi$ on the polynomial core $\mathcal{T}_{S}^{q}$ respects $H$ and permutes the $P_{j}$, it follows that the representation $\rho_{\varphi}$ is fixed under the action of $\varphi$. As above, this means that there exists an isomorphism $L_{\varphi}$ of $V$ such that

$$
\rho_{\varphi} \circ \varphi_{S}^{q}(X)=L_{\varphi} \cdot \rho_{\varphi}(X) \cdot L_{\varphi}^{-1}
$$


in $\operatorname{End}(V)$ for every $X \in \mathcal{T}_{S}^{q}$.

Theorem 40 Let $N$ be odd, and let $q$ be a primitive $N$-th root of 1 . The isomorphism $L_{\varphi}$ of $V$ defined above depends uniquely on $q$ and on the homotopically aperiodic diffeomorphism $\varphi: S \rightarrow S$, up to conjugation and up to scalar multiplication.

In particular, any invariant of $L_{\varphi}$ is an invariant of $\varphi$. For instance, we can consider the spectrum of $\varphi$ (consisting of $3 g+p-3$ non-zero complex numbers) up to scalar multiplication. Similarly, we can normalize the matrix $L_{\varphi}$ so that its determinant is equal to 1 ; its trace $\operatorname{Tr}\left(L_{\varphi}\right)$ then is a weaker invariant well-defined up to a root of unity. Another interesting invariant is $\operatorname{Tr}\left(L_{\varphi}\right) \operatorname{Tr}\left(L_{\varphi}^{-1}\right)$, which is the trace of the linear automorphism of $\operatorname{End}(V)$ defined by conjugation by $L_{\varphi}$.

See [26] for explicit computations of $L_{\varphi}$ for diffeomorphisms of the once-punctured torus and of the 4-times punctured sphere.

\section{References}

[1] H Bai, A uniqueness property for the quantization of Teichmüller spaces arXiv: math.GT/0509679

[2] H Bai, Quantum hyperbolic geometry in dimensions 2 and 3, $\mathrm{PhD}$ thesis, Univ. of Southern California (2006)

[3] H Bai, F Bonahon, X Liu, Local representations of the quantum Teichmüller space, in preparation

[4] S Baseilhac, R Benedetti, QHI, 3-manifolds scissors congruence classes and the volume conjecture, from: "Invariants of knots and 3-manifolds (Kyoto, 2001)", (T Ohtsuki, et al, editors), Geom. Topol. Monogr. 4 (2002) 13-28 MR2002600

[5] S Baseilhac, R Benedetti, Quantum hyperbolic invariants of 3-manifolds with PSL(2, C) -characters, Topology 43 (2004) 1373-1423 MR2081430

[6] S Baseilhac, R Benedetti, Classical and quantum dilogarithmic invariants of flat PSL(2, C) -bundles over 3-manifolds, Geom. Topol. 9 (2005) 493-569 MR2140989

[7] S Baseilhac, R Benedetti, Quantum hyperbolic geometry arXiv:math. GT/0611504

[8] F Bonahon, Shearing hyperbolic surfaces, bending pleated surfaces and Thurston's symplectic form, Ann. Fac. Sci. Toulouse Math. (6) 5 (1996) 233-297 MR1413855

[9] K A Brown, K R Goodearl, Lectures on algebraic quantum groups, Advanced Courses in Mathematics. CRM Barcelona, Birkhäuser, Basel (2002) MR1898492

[10] D Bullock, C Frohman, J Kania-Bartoszyńska, Topological interpretations of lattice gauge field theory, Comm. Math. Phys. 198 (1998) 47-81 MR1657365 
[11] R D Canary, D B A Epstein, P Green, Notes on notes of Thurston, from: "Analytical and geometric aspects of hyperbolic space (Coventry/Durham, 1984)", London Math. Soc. Lecture Note Ser. 111, Cambridge Univ. Press (1987) 3-92 MR903850

[12] L O Chekhov, V V Fock, Observables in 3D gravity and geodesic algebras, from: "Quantum groups and integrable systems (Prague, 2000)", Czechoslovak J. Phys. 50 (2000) 1201-1208 MR1806262

[13] P M Cohn, Skew fields, Theory of general division rings, Encyclopedia of Mathematics and its Applications 57, Cambridge University Press (1995) MR1349108

[14] A Fathi, F Laudenbach, V Poénaru, Travaux de Thurston sur les surfaces, Astérisque 66-67, Soc. Math. France, Paris (1979) MR568308 Séminaire Orsay, With an English summary

[15] V V Fock, Dual Teichmüller spaces arXiv:dg-ga/9702018

[16] V V Fock, L O Chekhov, Quantum Teichmüller spaces, Teoret. Mat. Fiz. 120 (1999) 511-528 MR1737362 English translation: Theoret. and Math. Phys. 120 (1999) $1245-1259$

[17] V Fock, A Goncharov, Moduli spaces of local systems and higher Teichmüller theory, Publ. Math. Inst. Hautes Études Sci. (2006) 1-211 MR2233852

[18] C Frohman, R Gelca, Skein modules and the noncommutative torus, Trans. Amer. Math. Soc. 352 (2000) 4877-4888 MR1675190

[19] C Frohman, R Gelca, W Lofaro, The A-polynomial from the noncommutative viewpoint, Trans. Amer. Math. Soc. 354 (2002) 735-747 MR1862565

[20] J L Harer, The virtual cohomological dimension of the mapping class group of an orientable surface, Invent. Math. 84 (1986) 157-176 MR830043

[21] R M Kashaev, A link invariant from quantum dilogarithm, Modern Phys. Lett. A 10 (1995) 1409-1418 MR1341338

[22] R M Kashaev, The hyperbolic volume of knots from the quantum dilogarithm, Lett. Math. Phys. 39 (1997) 269-275 MR1434238

[23] R M Kashaev, Quantization of Teichmüller spaces and the quantum dilogarithm, Lett. Math. Phys. 43 (1998) 105-115 MR1607296

[24] C Kassel, Quantum groups, Graduate Texts in Mathematics 155, Springer, New York (1995) MR1321145

[25] X Liu, The quantum Teichmüller space as a non-commutative algebraic object arXiv: math.GT/0408361

[26] X Liu, Quantum hyperbolic invariants for diffeomorphisms of small surfaces arXiv: math.GT/0603467

[27] G D Mostow, Strong rigidity of locally symmetric spaces, Annals of Mathematics Studies 78, Princeton University Press (1973) MR0385004 
[28] H Murakami, J Murakami, The colored Jones polynomials and the simplicial volume of a knot, Acta Math. 186 (2001) 85-104 MR1828373

[29] H Murakami, J Murakami, M Okamoto, T Takata, Y Yokota, Kashaev's conjecture and the Chern-Simons invariants of knots and links, Experiment. Math. 11 (2002) 427435 MR1959752

[30] J-P Otal, Le théorème d'hyperbolisation pour les variétés fibrées de dimension 3, Astérisque (1996) x+159 MR1402300

[31] A Papadopoulos, R C Penner, The Weil-Petersson symplectic structure at Thurston's boundary, Trans. Amer. Math. Soc. 335 (1993) 891-904 MR1089420

[32] R C Penner, The decorated Teichmüller space of punctured surfaces, Comm. Math. Phys. 113 (1987) 299-339 MR919235

[33] R C Penner, Weil-Petersson volumes, J. Differential Geom. 35 (1992) 559-608 MR1163449

[34] J H Przytycki, Skein modules of 3-manifolds, Bull. Polish Acad. Sci. Math. 39 (1991) $91-100$

[35] J H Przytycki, A S Sikora, On skein algebras and $\mathrm{Sl}_{2}(\mathbb{C})$-character varieties, Topology 39 (2000) 115-148 MR1710996

[36] Y Sözen, F Bonahon, The Weil-Petersson and Thurston symplectic forms, Duke Math. J. 108 (2001) 581-597 MR1838662

[37] W P Thurston, The topology and geomety of 3-manifolds, Lecture notes, Princeton University (1976-79)

[38] W P Thurston, Three-dimensional manifolds, Kleinian groups and hyperbolic geometry, Bull. Amer. Math. Soc. (N.S.) 6 (1982) 357-381 MR648524

[39] WP Thurston, Minimal stretch maps between hyperbolic surfaces, unpublished preprint (1986) arXiv:math.GT/9801039

[40] W P Thurston, On the geometry and dynamics of diffeomorphisms of surfaces, Bull. Amer. Math. Soc. (N.S.) 19 (1988) 417-431 MR956596

[41] W P Thurston, Three-dimensional geometry and topology. Vol. 1, Princeton Mathematical Series 35, Princeton University Press, Princeton, NJ (1997) MR1435975 Edited by S Levy

[42] V G Turaev, Skein quantization of Poisson algebras of loops on surfaces, Ann. Sci. École Norm. Sup. (4) 24 (1991) 635-704 MR1142906

[43] Y Yokota, On the volume conjecture for hyperbolic knots arXiv:math.QA/0009165

[44] Y Yokota, On the potential functions for the hyperbolic structures of a knot complement, from: "Invariants of knots and 3-manifolds (Kyoto, 2001)", (T Ohtsuki, et al, editors), Geom. Topol. Monogr. 4 (2002) 303-311 MR2002618 
Department of Mathematics, University of Southern California

Los Angeles, CA 90089-2532, USA

Department of Mathematics, Columbia University

2990 Broadway, New York, NY 10027, USA

fbonahon@math.usc.edu, xiaoboli@cpw.math.columbia.edu

Proposed: Jean-Pierre Otal

Seconded: Walter Neumann, Joan Birman
Received: 16 December 2005

Accepted: 13 December 2006 